\title{
Low Streamflow Conditions in Washington, Oregon, and Idaho During Water Year 2001
}

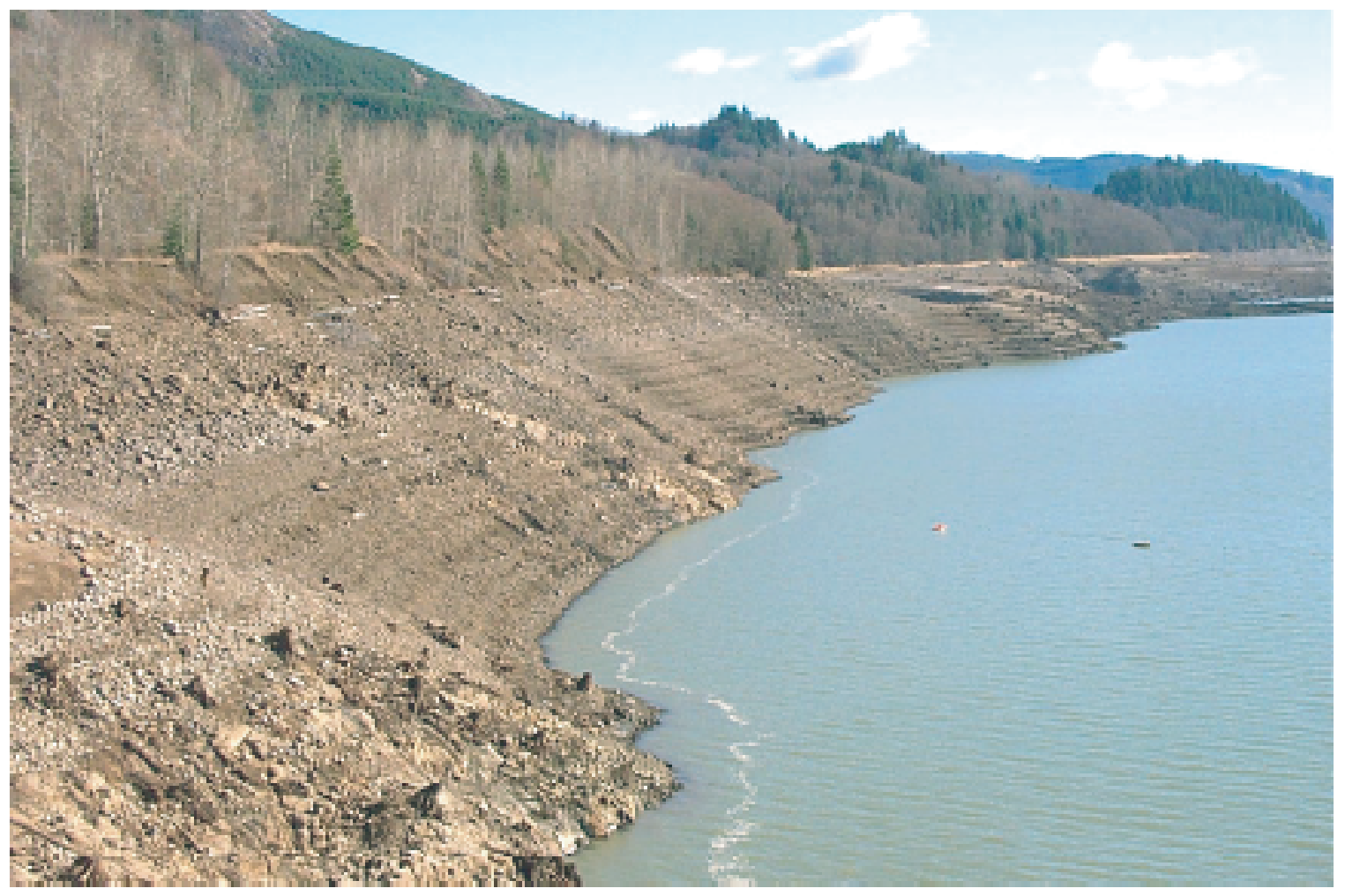


Cover: Riffle Lake near Mossyrock on the Cowlitz River, Washington, February 7, 2001. Water level is 117 to 125 feet below full (photo by John McKenna, U.S. Geological Survey). 


\section{Low Streamflow Conditions in Washington, Oregon, and Idaho During Water Year 2001}

By Jon E. Hortness

Water-Resources Investigations Report 03-4071

Boise, Idaho

2003 


\section{U.S. DEPARTMENT OF THE INTERIOR}

Gale A. Norton, Secretary

U.S. GEOLOGICAL SURVEY

Charles G. Groat, Director

Any use of firm, trade, and brand names in this report is for identification purposes only and does not constitute endorsement by the U.S. Government.

Additional information can be obtained from:

District Chief

U.S. Geological Survey

230 Collins Road

Boise, ID 83702-4520

http://idaho.usgs.gov
Copies of this report can be purchased from:

U.S. Geological Survey

Information Services

Box 25286

Federal Center

Denver, CO 80225

e-mail: infoservices@usgs.gov
Copies of this report also are available in PDF format, which can be viewed using Adobe Acrobat Reader, at URL: http://idaho.usgs.gov/public/reports.html 


\section{Contents}

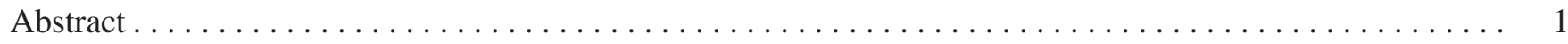

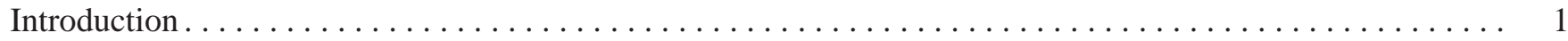

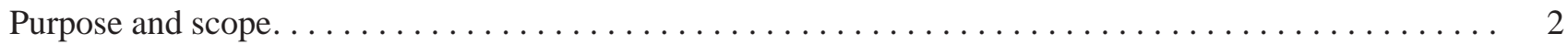

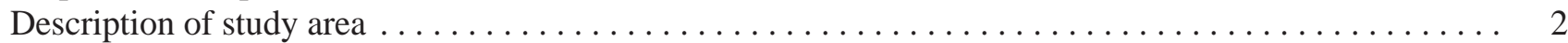

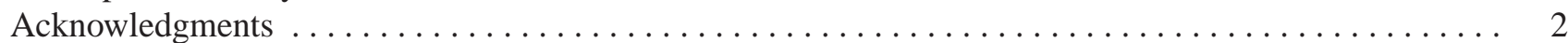

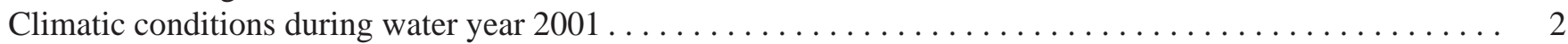

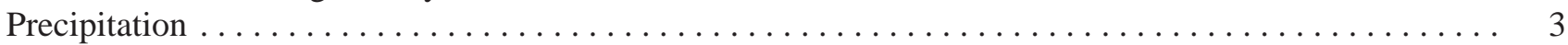

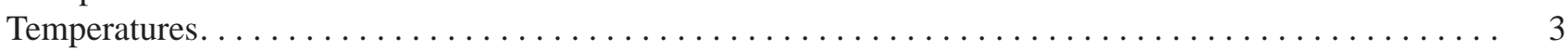

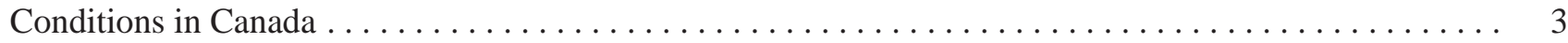

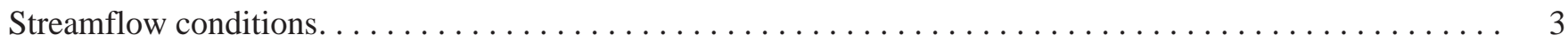

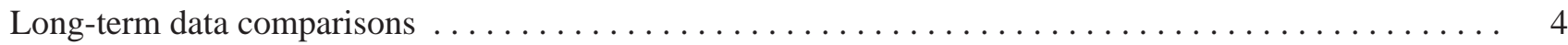

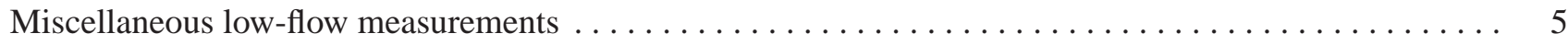

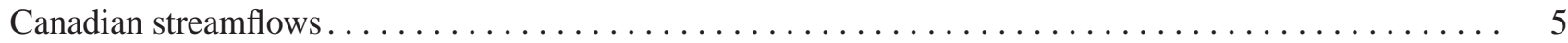

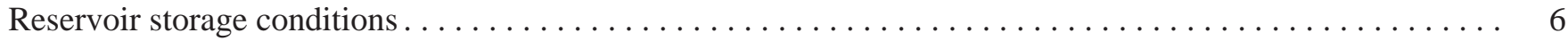

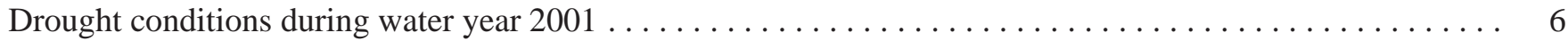

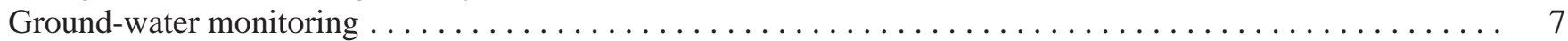

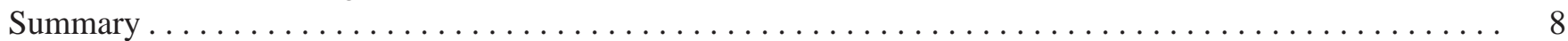

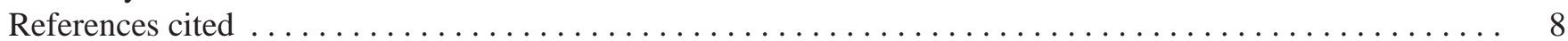

\section{FIGURES}

1-3. Maps showing:

1. Location of study area, major lakes, reservoirs, and rivers, Washington, Oregon,

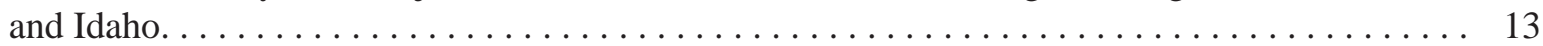

2. Location of major basins, Washington, Oregon, and Idaho $\ldots \ldots \ldots \ldots \ldots \ldots \ldots \ldots \ldots$

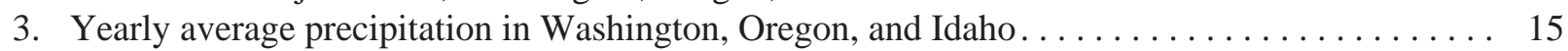

4. Graphs showing monthly precipitation during water year 2001, and monthly normal precipitation during 1961-90 at selected weather stations in Washington, Oregon, and Idaho . . . . . . . . . . 16

5. Map showing location of long-term gaging stations used as index sites, Washington, Oregon,

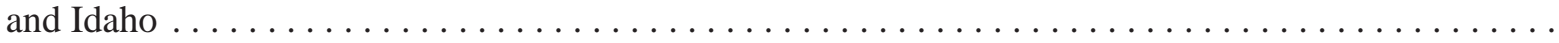

6-8. Graphs showing:

6. Daily mean streamflow for water years 1977 and 2001, and long-term minimum and median

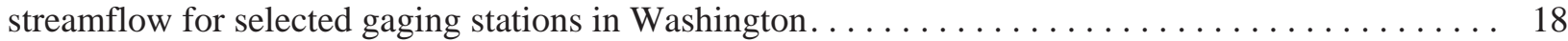

7. Daily mean streamflow for water years 1977 and 2001, and long-term minimum and median streamflow for selected gaging stations in Oregon $\ldots \ldots \ldots \ldots \ldots \ldots \ldots \ldots \ldots \ldots \ldots \ldots$

8. Daily mean streamflow for water years 1977 and 2001, and long-term minimum and median

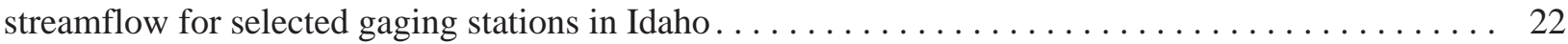

9-10. Maps showing:

9. Location of low-flow measurement sites, Washington, Oregon, and Idaho, water year 2001 . . . 24

10. Palmer Drought Severity Index map for the continental United States, September 1, 2001 . . . 25

11. Location of long-term monitoring wells, Washington, Oregon, and Idaho, water year 2002 . . . 26 


\section{TABLES}

1. Annual runoff volume comparisons for selected gaging stations in Washington, Oregon,

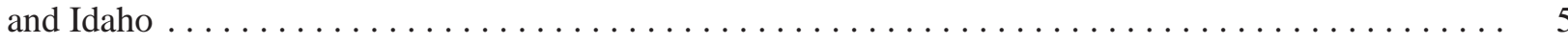

2. Low-flow measurement data for 410 selected sites in Washington, water years 1977

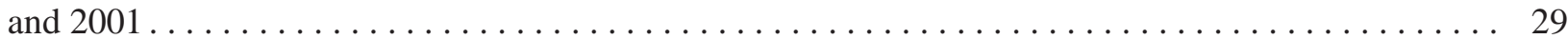

3. Low-flow measurement data for 166 selected sites in Oregon, water years 1977 and $2001 \ldots$. . . . . 43

4. Low-flow measurement data for 155 selected sites in Idaho, Wyoming, Utah, and Nevada,

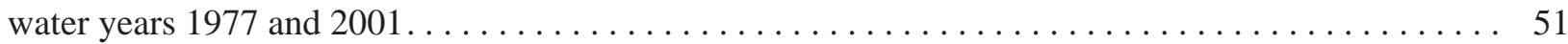

5. Water year 2001 year-end reservoir storage values and long-term averages for reservoirs in major river basins in Idaho, Wyoming, Oregon, Nevada, Washington, and California . . . . . . . 6

6. Site information and monitoring frequency for selected wells in Washington, Oregon,

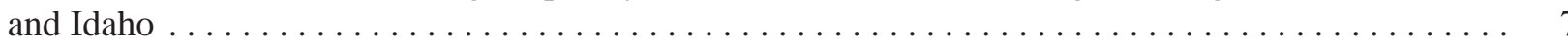

\section{CONVERSION FACTORS AND WATER YEAR DEFINITION}

\begin{tabular}{rcl}
\hline Multiply & By & To obtain \\
\hline acre-foot (acre-ft) & 1,233 & cubic meter $\left(\mathrm{m}^{3}\right)$ \\
cubic foot per second (ft $3 / \mathrm{s})$ & 0.02832 & cubic meter per second $\left(\mathrm{m}^{3} / \mathrm{s}\right)$ \\
inch (in.) & 2.54 & centimeter $(\mathrm{cm})$ \\
inch per year (in/yr) & 2.54 & centimeter per year $(\mathrm{cm} / \mathrm{yr})$ \\
mile $(\mathrm{mi})$ & 1.609 & kilometer $(\mathrm{km})$ \\
square mile $\left(\mathrm{mi}^{2}\right)$ & 2.590 & square kilometer $\left(\mathrm{km}^{2}\right)$ \\
\hline
\end{tabular}

Water year: In U.S. Geological Survey reports dealing with surface-water supply, a water year is the 12-month period, October 1 through September 30. The water year is designated by the calendar year in which it ends; thus, the water year ending September 30, 2001, is called the "2001 water year." 


\title{
Low Streamflow Conditions in Washington, Oregon, and Idaho During Water Year 2001
}

\author{
By Jon E. Hortness
}

\section{Abstract}

Below-normal precipitation levels and abovenormal temperatures across most of the Columbia River Basin in the Pacific Northwest (Washington, Oregon, and Idaho) resulted in streamflows that, at times, approached long-term minimums. The period from October 1, 2000, through September 30, 2001 (water year 2001), was the second driest on record (1895-2001) for the three-State area. In addition, average temperatures during the April through September 2001 period were the twelfth highest since 1895. Conditions in the part of Canada included in the Columbia River Basin were similar.

Streamflow levels at several locations approached those during water year 1977, when several minimum-flow records were set. The drought of 1977 commonly is considered the drought of record in the region. Low streamflows were most noticeable in rivers east of the Cascade Range, where most of the streamflow above base flow is a direct result of snowmelt runoff. Because of below-normal precipitation across the region, snowpack levels in the three States were only about 59 to 62 percent of the long-term (1961-90) average.

Miscellaneous low-flow measurements were made at more than 700 locations across the threeState region and in some adjacent States. These measurements were made in late summer and early fall of 2001 during base-flow conditions. In general, these low-flow measurements were similar to those made at the same locations during water year 1977.

Reservoir storage values for seven major river basins in the three-State region were all below the 30-year average at the end of water year 2001. Reservoir storages were at average levels at the end of water year 2000; thus, the below-aver- age levels in water year 2001 can be related directly to low streamflows during water year 2001.

Near the end of water year 2001, the Palmer Drought Severity Index ranked much of the region in the severe or extreme drought categories. Only the coastal area of Washington and Oregon and part of the mountain region in Idaho were in the near-normal category. The National Oceanic and Atmospheric Administration classified most of the area as exhibiting adverse agricultural, hydrological, and fire-danger effects from the drought.

Lack of available water for recharge and increased pumpage needed to augment the reduced surface-water supply likely reduced ground-water levels throughout the region. Twenty-five wells across the region were selected for extended monitoring to help define the possible short- and longterm relation between low streamflows and ground-water levels.

\section{INTRODUCTION}

Streamflow levels in Washington, Oregon, and Idaho during water year 2001 (WY01) were, in general, the lowest since WY77. Although no recurrence interval was calculated for the drought that encompassed the three-State area during 1977, it is commonly considered as the drought of record, and all-time low streamflows were recorded at many locations (Hubbard, 1987; Paulson and others, 1991). Late-summer base flows in a few rivers in WY01 were the lowest on record, and below-normal precipitation and above-normal temperatures were common across most of the Pacific Northwest and western Canada. For the purpose of this report, the term "normal" refers to the 30-year (1961-90) average precipitation or temperature as defined by the National Climatic Data Center. Low precipitation levels during the winter months resulted in snowpacks that were well below average across the region. Water levels in many of the reservoirs in the 
region were also below the long-term averages, which created problems with adequate supplies of water to satisfy hydroelectric, recreational, and irrigation purposes, as well as fish and wildlife habitat needs. Major power companies raised rates following significant power shortages that resulted from below-normal hydroelectric power generation across the Western United States, most notably in California. In addition, recreational, agricultural, and biological interest groups waged public and sometimes legal battles while attempting to secure the rights to any available water. In the end, many reservoirs and rivers were too low for most recreational uses, irrigation was halted in several areas, and fish and wildlife suffered from lack of available water. Drought emergencies were declared throughout most of the three-State area. Low streamflow data are important to water-management agencies, interest groups, and the public. To adequately monitor and document the low streamflows during WY01, the Washington, Oregon, and Idaho Districts of the U.S. Geological Survey collected more than 700 miscellaneous streamflow measurements across the region and summarized long-term hydrologic and climatic data. In addition, 25 wells across the region were selected for extended monitoring to help define the possible shortand long-term effects of low streamflows on groundwater levels.

\section{Purpose and Scope}

The purpose of this report is to (1) document low streamflows at several ungaged locations on streams in Washington, Oregon, and Idaho; (2) provide comparisons between WY01 and historical streamflow data at several long-term gaging stations; and (3) relate miscellaneous low-flow measurements from WY01 to measurements from WY77. Precipitation and temperature data are used for comparing WY01 data to longterm data. The effects of low streamflows on reservoir storages and ground-water levels also are discussed.

\section{Description of Study Area}

The States of Washington, Oregon, and Idaho make up the area of study for this report (figure 1, back of report). Most of this area is included in the Columbia River Basin, and smaller areas are part of various Pacific Slope Basins; landlocked basins in Oregon (including Silver Lake, Lake Albert, Goose Lake,
Harney Lake, and Malheur Lake); and the Great Basin (figure 2, back of report). The Columbia River Basin is highly regulated for hydroelectric, irrigation, and flood-control purposes. Parts of the other basins also are regulated to some extent.

The Cascade Range trends north-south through Washington and Oregon and provides a natural division between the western and eastern areas of each State. The climate west of the Cascade Range is characterized by moderate temperatures, wet winters, and dry summers. Parts of western slopes in Washington's Olympic Mountains receive as much as $200 \mathrm{in} / \mathrm{yr}$ of precipitation (figure 3, back of report); 120 to $150 \mathrm{in} / \mathrm{yr}$ of precipitation is common across the rest of the Cascade Range. Moderate to cold temperatures are prevalent in areas of Washington and Oregon east of the Cascade Range; however, a general lack of moisture in cloud masses after crossing the mountains commonly causes dry winters and very dry summers. The lowelevation areas east of the Cascade Range generally receive between 10 and $40 \mathrm{in} / \mathrm{yr}$; high-elevation areas in northeastern Oregon receive as much as $80 \mathrm{in} / \mathrm{yr}$. Idaho's climate is similar to that of eastern Washington and Oregon. Moderate to cold temperatures are common throughout the State, and precipitation ranges from 10 to $30 \mathrm{in} / \mathrm{yr}$ in the low elevations to as much as $70 \mathrm{in} / \mathrm{yr}$ in the central mountains.

\section{Acknowledgments}

The data-collection efforts of field office personnel in the Washington, Oregon, and Idaho Districts are greatly appreciated. The surface-water data sections in each District also provided assistance with data compilation and record keeping and are gratefully acknowledged.

\section{CLIMATIC CONDITIONS DURING WATER YEAR 2001}

Low streamflow conditions are generally a direct result of below-normal precipitation and often are made worse by above-normal temperatures. Lower streamflows also can result from residual effects of previous low-water years. The effects of reduced groundwater recharge might not be felt for some time and, in highly regulated basins with large reservoir storages, reduced reservoir levels will affect streamflows during future years. It is important to note that low stream- 
flows in the Columbia River system also can be affected by conditions in the part of Canada that is included in the Columbia River Basin. About 15 percent $\left(39,000 \mathrm{mi}^{2}\right)$ of the Columbia River Basin is in Canada.

\section{Precipitation}

Most of the annual precipitation in the Pacific Northwest occurs during the months of November through March. The precipitation is typically in the form of snow across much of the region, with the exception of the low-elevation Pacific Slope Basins, and the resulting snowpack generally melts off during the months of April, May, and June. Precipitation levels prior to WY01 were near average across most of the study area. Water year 2000 precipitation levels across Washington ranged from 90 to 110 percent of normal (1961-90); the ranges for Oregon and Idaho were 85 to 112 percent and 78 to 102 percent of normal, respectively (Natural Resources Conservation Service, Precipitation Reports, 2002). In addition, with the exception of parts of central and southwestern Oregon, precipitation levels in September 2000 ranged from normal to well above normal.

The period November 2000 through March 2001, however, was the second driest on record (1895-2001); the average regional precipitation level was approximately 8.5 in. Only the 1976-77 period was drier, with an average of about $7.5 \mathrm{in}$. The normal November through March precipitation level for the region was nearly 17 in. (National Oceanic and Atmospheric Administration, National Climatic Data Center, 2002). As a result of below-average precipitation during the winter months, snowpack levels across the region were also well below average. Average snowpack levels in Washington on April 1, 2001, were 59 percent of the 30-year average (1961-1990); Idaho, 61 percent of average; and Oregon, 62 percent of average. Snowpack levels in parts of northeastern Washington, southern Oregon, and central Idaho were less than 50 percent of average (Natural Resources Conservation Service, Snowpack Reports, 2002).

Precipitation levels for the remainder of the water year (May through September) were much improved but still below normal across most of the region. Overall, WY01 was the second driest on record (1895-2001) for the Pacific Northwest and the second, third, and seventh driest for Washington, Oregon, and Idaho, respectively (National Oceanic and Atmospheric Administration, National Climatic Data Center, 2002). Comparisons between monthly precipitation levels for WY01 and normal monthly precipitation levels are presented in figure 4 (back of report) for selected sites across the study area. Well below-normal precipitation during November through March is evident in each of the precipitation plots.

\section{Temperatures}

Above-normal temperatures were prevalent across the three-State region during April through September 2001. The average temperature of the region was the twelfth highest since 1895. Idaho experienced the largest deviation from normal. Average temperatures for the April through September period were the fifth highest on record, and August average temperatures were the highest ever recorded in Idaho (1895-2001) (National Oceanic and Atmospheric Administration, National Climatic Data Center, 2002).

\section{Conditions in Canada}

Climatic conditions in the part of Canada included in the Columbia River Basin were similar to those in the three-State region during WY01. The accumulated precipitation since the beginning of November was about 50 percent of normal (1935-2001) for the Columbia River Basin. Snowpacks on April 1, 2001, in major subbasins of the Columbia River Basin in Canada ranged from 45 to 68 percent of normal. Temperatures across the region were also above normal, and average temperatures for the entire country were third highest on record (1935-2001). Despite cooler temperatures in early June that delayed snowmelt, snowpack levels were still well below average (Government of British Columbia, 2002).

\section{STREAMFLOW CONDITIONS}

WY01 began with near-average streamflows in most streams in the Pacific Northwest. Although streamflows increased in Pacific Slope Basin streams during the winter months, these flows were well below average. Snowmelt runoff resulted in increased flows during the spring months, but again, these flows were well below average. Streamflows stayed below average 
across most of the region for the remainder of the water year.

\section{Long-Term Data Comparisons}

Eighteen continuous-recording gaging stations were selected as index sites for use in this report. The sites (figure 5, back of report) were selected on the basis of several factors: location, size of the basin, length of record, and absence of significant diversions or regulations. Plots of daily mean streamflow for WY01 in relation to the WY77 daily mean, long-term minimum, and long-term median streamflow for each site are presented in figure 6 , figure 7 , and figure 8 (back of report).

Streamflows at index site 1 in Washington (figure 6) and sites 7 and 8 in Oregon (figure 7) originate in Pacific Slope Basins that receive large amounts of rainfall and little or no snowfall. Thus, streamflows are typically high during the wet-season months of November through March. In general, streamflows at these sites in WY01 were below the long-term median, with the exception of several instances when rainfall resulted in short-duration peaks that were higher than the long-term median. Compared with WY77, coastal rainfall in WY01 was generally smaller in magnitude; however, individual storms were more numerous and resulted in more cumulative streamflow.

Streamflows at sites 2, 3, and 9 (figure 6 and figure 7) originate in high-elevation Pacific Slope Basins and are affected by wet-season rains and spring snowmelt (April through June). Streamflows are typically higher than those in comparable inland streams during the wet season as a result of rainfall and traditionally peak in the spring as a result of snowmelt runoff from high elevations. As was the case with the coastal sites, streamflows at these sites were well below the long-term median, with the exception of several short-duration peaks resulting from rainfall. In addition, typical peak flow resulting from snowmelt runoff was insignificant, likely a result of below-average snowpack at high elevations because of below-normal precipitation in the preceding months. Conditions were similar to those in WY77 when streamflows were below the long-term median and increases in streamflow resulting from snowmelt were insignificant.

The remaining index sites (4-6, Washington, figure $6 ; 10-12$, Oregon, figure $7 ; 13-18$, Idaho, figure 8) are all east of the Cascade Range. Most of the streamflow above base flow at these sites is a direct result of snowmelt runoff during the spring or early summer months. Rainfall in this area contributes significantly less streamflow on an annual basis but, over a short period of time, can result in significant flow increases and possible flooding. Streamflows at some of these sites, most notably, sites 4, 6, and 12, were slightly above the long-term median at the beginning of WY01 but, in general, were below median values during most of the water year. Lack of available snowpack, exhibited by short-duration and low-magnitude spring runoff, is evident in the hydrographs for nearly every site. With few exceptions, increased runoff from snowmelt diminished by early June and, in many cases, streamflows approached long-term minimums. Site 12 in southeastern Oregon is the only site where streamflows were near the long-term median values for much of the snowmelt season.

Compared with WY77, streamflows resulting from snowmelt in WY01 were generally higher, with few exceptions. In many cases, the WY77 streamflows were at or near long-term minimums during much of the snowmelt season, with little, if any, increases from snowmelt. In contrast, streamflows at most sites in WY01 exhibited some form of runoff peak, albeit much lower than average.

Additional evidence of below-average streamflow in WY01 can be seen in the annual runoff data. Total annual runoff volumes at each of the index sites for WY01 compared with WY77 and long-term averages are presented in table 1.

As expected, the annual runoff volumes for WY01 were also well below the long-term averages. Values for the index sites across the three-State area ranged from 34 percent (Chetco River, Oregon) to 70 percent (Umatilla River, Oregon) of average. Runoff at only three of the index sites was lower in WY01 than in WY77 (Sauk River, Washington; Wilson River, Oregon; and Big Lost River, Idaho). However, at two of those sites (Sauk River, Washington; and Wilson River, Oregon), the WY01 value was the minimum for the period of record.

\section{Miscellaneous Low-Flow Measurements}

Streamflow was measured at 724 miscellaneous sites across the three-State region and at an additional 
Table 1. Annual runoff volume comparisons for selected gaging stations in Washington, Oregon, and Idaho [WA, Washington; OR, Oregon; ID, Idaho; WY, Wyoming]

\begin{tabular}{|c|c|c|c|c|c|c|c|c|}
\hline \multirow[b]{2}{*}{$\begin{array}{c}\text { Map } \\
\text { No. } \\
\text { (fig. 3) }\end{array}$} & \multirow[b]{2}{*}{$\begin{array}{c}\text { Gaging- } \\
\text { station } \\
\text { No. }\end{array}$} & \multirow[b]{2}{*}{ Gaging-station name } & \multirow[b]{2}{*}{$\begin{array}{l}\text { Period } \\
\text { of } \\
\text { record }\end{array}$} & \multirow[b]{2}{*}{$\begin{array}{c}\text { Long-term } \\
\text { average } \\
\text { annual } \\
\text { runoff } \\
\text { volume } \\
\text { (acre-ft) }\end{array}$} & \multicolumn{2}{|c|}{ Water year 2001} & \multicolumn{2}{|c|}{ Water year 1977} \\
\hline & & & & & $\begin{array}{c}\text { Annual } \\
\text { runoff } \\
\text { volume } \\
\text { (acre-ft) }\end{array}$ & $\begin{array}{c}\text { Percent } \\
\text { of long- } \\
\text { term } \\
\text { average/ } \\
\text { (long- } \\
\text { term } \\
\text { ranking }^{1} \text { ) }\end{array}$ & $\begin{array}{c}\begin{array}{c}\text { Annual } \\
\text { runoff } \\
\text { volume } \\
\text { (acre-ft) }\end{array} \\
\end{array}$ & $\begin{array}{c}\text { Percent } \\
\text { of long- } \\
\text { term } \\
\text { average/ } \\
\text { (long- } \\
\text { term } \\
\text { ranking }^{1} \text { ) }\end{array}$ \\
\hline 1 & 12035000 & Satsop River near Satsop, WA & $1929-2001$ & $1,478,000$ & 922,700 & $62 /(4)$ & 868,200 & $59 /(1)$ \\
\hline 2 & 12048000 & Dungeness River near Sequim, WA & 1923-2001 & 278,900 & 150,900 & $54 /(3)$ & 142,700 & $51 /(1)$ \\
\hline 3 & 12186000 & $\begin{array}{l}\text { Sauk River above Whitechuck River } \\
\text { near Darrington, WA }\end{array}$ & $1918-22,1929-2001$ & 815,100 & 457,100 & $56 /(1)$ & 542,500 & $67 /(4)$ \\
\hline 4 & 12488500 & American River near Nile, WA & 1940-2001 & 169,800 & 78,480 & $46 /(2)$ & 68,200 & $40 /(1)$ \\
\hline 5 & 13351000 & Palouse River at Hooper, WA & $1898-1916,1951-2001$ & 441,900 & 189,600 & $43 /(9)$ & 76,610 & $17 /(1)$ \\
\hline 6 & 12431000 & Little Spokane River at Dartford, WA & $1929-32,1947-2001$ & 220,000 & 144,700 & $66 /(8)$ & 112,000 & $51 /(4)$ \\
\hline 7 & 14301500 & Wilson River near Tillamook, OR & $1915-16,1931-2001$ & 853,300 & 358,300 & $42 /(1)$ & 379,600 & $44 /(2)$ \\
\hline 8 & 14400000 & Chetco River near Brookings, OR & 1970-2001 & $1,627,000$ & 557,500 & $34 /(2)$ & 397,800 & $24 /(1)$ \\
\hline 9 & 14178000 & $\begin{array}{l}\text { North Santiam River below Boulder } \\
\text { Creek near Detroit, OR }\end{array}$ & $1907-10,1929-2001$ & 728,800 & 431,300 & $59 /(2)$ & 412,300 & $57 /(1)$ \\
\hline 10 & 14046500 & John Day River at Service Creek, OR & $1930-2001$ & $1,408,000$ & 635,000 & $45 /(10)$ & 448,400 & $32 /(1)$ \\
\hline 11 & 10396000 & $\begin{array}{l}\text { Donner und Blitzen River near } \\
\text { Frenchglen, OR }\end{array}$ & $\begin{array}{c}1911-21,1929-30 \\
1938-2001\end{array}$ & 92,130 & 59,960 & $65 /(11)$ & 48,300 & $52 /(6)$ \\
\hline 12 & 14020000 & $\begin{array}{l}\text { Umatilla River above Meacham } \\
\text { Creek near Gibbon, OR }\end{array}$ & 1933-2001 & 164,200 & 114,200 & $70 /(8)$ & 82,300 & $50 /(1)$ \\
\hline 13 & 12414500 & St. Joe River at Calder, ID & 1911-12, 1920-2001 & $1,694,000$ & 814,600 & $48 /(3)$ & 798,700 & $47 /(2)$ \\
\hline 14 & 13317000 & Salmon River at White Bird, ID & $1910-17,1920-2001$ & $8,085,000$ & $4,450,000$ & $55 /(3)$ & $4,214,000$ & $52 /(2)$ \\
\hline 15 & 13313000 & Johnson Creek at Yellow Pine, ID & $1928-2001$ & 249,000 & 99,860 & $40 /(2)$ & 88,920 & $36 /(1)$ \\
\hline 16 & 13185000 & Boise River near Twin Springs, ID & 1911-2001 & 867,300 & 430,900 & $50 /(4)$ & 320,400 & $37 /(1)$ \\
\hline 17 & 13120500 & $\begin{array}{l}\text { Big Lost River at Howell Ranch, near } \\
\text { Chilly, ID }\end{array}$ & $1949-2001$ & 229,700 & 115,400 & $50 /(2)$ & 130,700 & $57 /(6)$ \\
\hline 18 & 13023000 & $\begin{array}{l}\text { Greys River above Reservoir, near } \\
\text { Alpine, WY }\end{array}$ & $1937-39,1954-2001$ & 468,700 & 274,000 & $58 /(3)$ & 187,400 & $40 /(1)$ \\
\hline
\end{tabular}

${ }^{1}$ Overall ranking for the period of record from minimum to maximum (ranking of 1 is the all-time minimum).

7 sites in Nevada, Utah, and Wyoming. These 731 measurements were made during base-flow conditions in late summer or early fall of 2001. The spatial extent of the measurement locations is shown in figure 9 (back of report) and the low-flow measurement data are presented in table 2, table 3, and table 4 (back of report) for Washington, Oregon, and Idaho, respectively. It is apparent that the values for low-flow measurements made in WY01 are generally similar to those made in WY77. Thus, although the timing of measurements was different at many sites, the low flows measured in WY01 generally are in the same range as those measured in WY77.

\section{Canadian Streamflows}

Because about 15 percent of the Columbia River Basin is in Canada, low streamflows in the Columbia River system in the United States also can be affected by conditions in the part of Canada that is included in the Columbia River Basin. Runoff was well below average in the Columbia River Basin. Runoff was well below average in the Columbia River Basin in Canada, and many streams reached their annual peak levels prior to June 1, 2001. In addition, streamflows in the Kootenay River in Canada were only 53 percent of average for the year (Government of British Columbia, 2002). 


\section{RESERVOIR STORAGE CONDITIONS}

Depending on the time and spatial extent of below-average streamflows, both short-term and longterm effects with regard to reservoir storages can be noticeable. A summary of WY01 year-end storage values compared with long-term average storage values for reservoirs in seven major river basins in the Pacific Northwest is presented in table 5 (Natural Resources Conservation Service, Reservoir Reports, 2002). As discussed previously, precipitation levels for several years prior to WY01 were at or above normal, resulting in average to above-average streamflows and reservoir storages. Thus, the below-average reservoir storage can be related directly to low streamflows during WY01. Compared with the 30-year average storage from 1961 through 1990, reservoir storage at the end of WY01 for the major basins listed in table 5 ranged from 17 to 92 percent of average.

The large variance in reservoir storage values likely can be attributed to varying management practices for each reservoir. Factors that influence how a reservoir is managed include hydroelectric potential, flood-control ability, recreational use, irrigation needs, and fish and wildlife habitat needs.

\section{DROUGHT CONDITIONS DURING WATER YEAR 2001}

The National Oceanic and Atmospheric Administration (NOAA) uses the Palmer Drought Severity Index (PDSI) as one measure of the intensity, duration, and spatial extent of a drought. NOAA also uses a multi-index drought classification system to summarize the primary physical effects of a drought. This system divides the primary physical effects of a drought into three categories: (1) agricultural (crops and livestock); (2) hydrological (rivers, ground water, reservoirs, and water supplies); and (3) fire danger.

A map showing the PDSI values for the continental United States on September 1, 2001, is presented in figure 10 (back of report) (National Oceanic and Atmospheric Administration, 2001). The values for the index are derived from measurements of precipitation, air temperature, and local soil moisture. These values have been standardized by region to allow for accurate comparisons. Although the determination of the index does not make it suitable for estimating long-term hydrologic droughts that can impact streamflows, the map in figure 10 does represent the conditions in the region near the end of the 2001 water year. At that time, nearly all of Idaho and Oregon and more than half of Washington were classified as being under severe or extreme drought conditions. Only in the coastal areas in Oregon and Washington and a high mountain region in Idaho were conditions classified as being near normal. It also is apparent that severe drought conditions extended into other Western States, including Montana, Wyoming, Utah, Nevada, and California. Conditions over much of the remainder of the country were generally near normal.

Even though the PDSI listed conditions in the coastal areas of Washington and Oregon as near normal, NOAA still classified this area as exhibiting adverse hydrological effects of the drought. This classification likely was the result of continuing belowaverage streamflows and reservoir levels. The remainder of the three-State region also exhibited adverse

Table 5. Water year 2001 year-end reservoir storage values and long-term averages for reservoirs in major river basins in Idaho, Wyoming, Oregon, Nevada, Washington, and California

[No., number; ft, feet; ID, Idaho; WY, Wyoming; OR, Oregon; NV, Nevada; WA, Washington; CA, California]

\begin{tabular}{|c|c|c|c|c|}
\hline \multirow[b]{2}{*}{ Basin } & \multirow[b]{2}{*}{$\begin{array}{c}\text { No. of } \\
\text { reservoirs }\end{array}$} & \multicolumn{2}{|c|}{ Storage contents, in acre-ft } & \multirow[b]{2}{*}{$\begin{array}{c}\text { Percent of } \\
\text { average }\end{array}$} \\
\hline & & $\begin{array}{c}\text { September } 30, \\
2001\end{array}$ & $\begin{array}{c}\text { Long-term } \\
\text { average }^{1}\end{array}$ & \\
\hline Upper Snake River Basin (ID, WY) & 8 & 668,300 & $2,322,700$ & 29 \\
\hline Boise and Payette River Basins (ID) & 7 & 633,000 & $1,080,400$ & 59 \\
\hline Owyhee and Malheur River Basins (OR, ID, NV) & 4 & 93,600 & 465,900 & 20 \\
\hline Upper Deschutes and Crooked River Basins (OR) & 5 & 222,200 & 240,500 & 92 \\
\hline Upper Yakima River Basin (WA) & 3 & 58,000 & 341,200 & 17 \\
\hline Willamette River Basin (OR) & 13 & 380,800 & 950,500 & 40 \\
\hline Klamath River Basin (OR, CA) & 3 & 353,200 & 523,500 & 67 \\
\hline
\end{tabular}

${ }^{1}$ Based on year-end values for 30-year period, 1961-90 (Natural Resources Conservation Service, Reservoir Reports, 2002). 
agricultural, hydrologic, and fire effects, according to the NOAA, U.S. Drought Monitor (2002).

\section{GROUND-WATER MONITORING}

Below-average streamflows can influence ground-water levels in local and regional aquifers. Ground-water levels in local alluvial aquifers can decline rapidly in response to below-average streamflow conditions, whereas levels in large regional aquifers might or might not be affected, depending on the time and spatial extent of low streamflow conditions. In addition, aquifers can be subject to increased pumpage necessary to augment decreases in the surfacewater supply. Conversely, water stored in aquifers sustains base flow; therefore, ground-water discharge can ameliorate streamflow. If recharge is lessened as a result of drought, there may be a delayed response of reduced base flow. Monthly ground-water data are inadequate to describe the effect of the 2001 drought on ground water in storage.

Twenty-five wells across the study area (figure 11, back of report) were selected in WY02 to monitor the possible short- and long-term effects of low streamflows on ground-water levels. Data will be collected on at least a bimonthly basis, and several of the wells are equipped with real-time systems. Well selection was based on the following criteria: (1) located in aquifers stressed by both pumping and drought, in areas potentially affecting many people; (2) currently being measured at least seasonally and having a relatively long period of record; and (3) geographically distributed

Table 6. Site information and monitoring frequency for selected wells in Washington, Oregon, and Idaho

[No., number; latitude and longitude are based on the North American Datum of 1983 and shown in degrees, minutes, seconds; WA, Washington; OR, Oregon; ID, Idaho]

\begin{tabular}{|c|c|c|c|c|c|}
\hline $\begin{array}{l}\text { Map No. } \\
\text { (fig. 11) }\end{array}$ & Well site No. & State & Latitude & Longitude & $\begin{array}{c}\text { Monitoring } \\
\text { frequency }\end{array}$ \\
\hline 1 & 465033122570202 & WA & $46^{\circ} 50^{\prime} 32^{\prime \prime}$ & $122^{\circ} 57^{\prime} 38^{\prime \prime}$ & real-time ${ }^{1}$ \\
\hline 2 & 472121122442011 & WA & $47^{\circ} 21^{\prime} 20^{\prime \prime}$ & $122^{\circ} 44^{\prime} 24^{\prime \prime}$ & continuous \\
\hline 3 & 462913119362102 & WA & $46^{\circ} 29^{\prime} 12^{\prime \prime}$ & $119^{\circ} 36^{\prime} 24^{\prime \prime}$ & monthly \\
\hline 4 & 473442118162201 & WA & $47^{\circ} 34^{\prime} 40^{\prime \prime}$ & $118^{\circ} 16^{\prime} 31^{\prime \prime}$ & monthly \\
\hline 5 & 461935118081501 & WA & $46^{\circ} 19^{\prime} 33^{\prime \prime}$ & $118^{\circ} 08^{\prime} 17^{\prime \prime}$ & monthly \\
\hline 6 & 470045117211701 & WA & $47^{\circ} 00^{\prime} 44^{\prime \prime}$ & $117^{\circ} 21^{\prime} 20^{\prime \prime}$ & monthly \\
\hline 7 & 474011117072901 & WA & $47^{\circ} 40^{\prime} 10^{\prime \prime}$ & $117^{\circ} 07^{\prime} 32^{\prime \prime}$ & monthly \\
\hline 8 & 441508123053001 & OR & $44^{\circ} 15^{\prime} 07^{\prime \prime}$ & $123^{\circ} 05^{\prime} 34^{\prime \prime}$ & monthly \\
\hline 9 & 420825123040401 & OR & $42^{\circ} 08^{\prime} 24^{\prime \prime}$ & $123^{\circ} 04^{\prime} 08^{\prime \prime}$ & monthly \\
\hline 10 & 444956123031701 & OR & $44^{\circ} 49^{\prime} 55^{\prime \prime}$ & $123^{\circ} 03^{\prime} 21^{\prime \prime}$ & monthly \\
\hline 11 & 452033122195901 & OR & $45^{\circ} 20^{\prime} 32^{\prime \prime}$ & $122^{\circ} 20^{\prime} 03^{\prime \prime}$ & monthly \\
\hline 12 & 442242121405501 & OR & $44^{\circ} 22^{\prime} 41^{\prime \prime}$ & $121^{\circ} 41^{\prime} 03^{\prime \prime}$ & real-time ${ }^{1}$ \\
\hline 13 & 434400121275801 & OR & $43^{\circ} 43^{\prime} 59^{\prime \prime}$ & $121^{\circ} 28^{\prime} 02^{\prime \prime}$ & real-time ${ }^{1}$ \\
\hline 14 & 475439116503401 & ID & $47^{\circ} 54^{\prime} 38^{\prime \prime}$ & $116^{\circ} 50^{\prime} 38^{\prime \prime}$ & bimonthly \\
\hline 15 & 443736116263701 & ID & $44^{\circ} 37^{\prime} 36^{\prime \prime}$ & $116^{\circ} 26^{\prime} 40^{\prime \prime}$ & real-time ${ }^{1}$ \\
\hline 16 & 444351116030001 & ID & $44^{\circ} 44^{\prime} 35^{\prime \prime}$ & $116^{\circ} 03^{\prime} 20^{\prime \prime}$ & monthly \\
\hline 17 & 431242115485501 & ID & $43^{\circ} 12^{\prime} 42^{\prime \prime}$ & $115^{\circ} 48^{\prime} 57^{\prime \prime}$ & bimonthly \\
\hline 18 & 424523115451201 & ID & $42^{\circ} 45^{\prime} 23^{\prime \prime}$ & $115^{\circ} 45^{\prime} 15^{\prime \prime}$ & real-time ${ }^{1}$ \\
\hline 19 & 424353114494701 & ID & $42^{\circ} 43^{\prime} 52^{\prime \prime}$ & $114^{\circ} 49^{\prime} 49^{\prime \prime}$ & monthly \\
\hline 20 & 423206113542301 & ID & $42^{\circ} 32^{\prime} 05^{\prime \prime}$ & $113^{\circ} 54^{\prime} 25^{\prime \prime}$ & bimonthly \\
\hline 21 & 432854113201002 & ID & $43^{\circ} 28^{\prime} 54^{\prime \prime}$ & $113^{\circ} 20^{\prime} 12^{\prime \prime}$ & bimonthly \\
\hline 22 & 423837113134302 & ID & $42^{\circ} 38^{\prime} 36^{\prime \prime}$ & $113^{\circ} 13^{\prime} 45^{\prime \prime}$ & monthly \\
\hline 23 & 432700112470801 & ID & $43^{\circ} 27^{\prime} 01^{\prime \prime}$ & $112^{\circ} 47^{\prime} 11^{\prime \prime}$ & bimonthly \\
\hline 24 & 440058112293601 & ID & $44^{\circ} 00^{\prime} 57^{\prime \prime}$ & $112^{\circ} 29^{\prime} 39^{\prime \prime}$ & bimonthly \\
\hline 25 & 440353112135701 & ID & $44^{\circ} 03^{\prime} 52^{\prime \prime}$ & $112^{\circ} 13^{\prime} 59^{\prime \prime}$ & monthly \\
\hline
\end{tabular}


across the region. Site information for the selected wells, including monitoring frequency, is given in table 6 . The ground-water data will be an invaluable resource in the future for assisting hydrologists and water managers in understanding and planning for reduced ground-water resources resulting, in part, from low-water years.

\section{SUMMARY}

Below-normal precipitation and above-normal temperatures were common across most of the Pacific Northwest and western Canada during water year 2001 (WY01). Especially low precipitation levels during the winter months resulted in below-average snowpacks across the region. As a result, streamflow levels at several locations in the Pacific Northwest during WY01 were significantly lower than the long-term median values for much of the year. Late-summer base flows in a few rivers were the lowest on record. Low streamflows were most noticeable in rivers east of the Cascade Range where most of the streamflow above base flow is a direct result of snowmelt runoff.

Compared with WY77, commonly considered as the drought of record, the magnitude of peak flows at most sites in WY01 was higher (although much lower than average), and total annual flow volumes in WY01 were generally only slightly higher than in WY77. Although the timing of miscellaneous low-flow measurements at any given location can vary, the values show that late-summer base flows were similar in WY77 and WY01.

Reservoir storage values for seven major river basins in the three-State region were all below the 30year (1961-90) average at the end of WY01. Reservoir storages were at average to above-average levels for several years prior to WY01; thus, the below-average levels in WY01 can be related directly to low streamflows during WY01.

Near the end of WY01, the Palmer Drought Severity Index ranked much of the region in the severe or extreme drought categories. Only the coastal area of Washington and Oregon and part of the mountain region in Idaho were in the near-normal category. The National Oceanic and Atmospheric Administration classified most of the area as exhibiting adverse agri- cultural, hydrological, and fire-danger effects from the drought.

The effects that the low streamflows of WY01 have on ground-water levels in local and regional aquifers might not be noticeable for several years. However, lack of available water for recharge and increased pumpage needed to augment the reduced surface-water supply likely reduced ground-water levels throughout the region. Twenty-five wells across the region were selected for extended monitoring to help define the possible short- and long-term effects of low streamflows on ground-water levels.

\section{REFERENCES CITED}

Daly, C., and Taylor, G., 1998, Western United States average monthly or annual precipitation, 196190, Oregon: Portland, Water and Climate Center of the Natural Resources Conservation Service, grid-cell resolution $4 \mathrm{~km}$, accessed May 2002, at URL

http://www.ocs.orst.edu/prism/prism_products.html

Government of British Columbia, River Forecast Centre-Archives, Snowpack and water supply outlook for British Columbia, April 1, 2001, accessed May 2002, at URL

http://srmwww.gov.bc.ca/aib/wat/rfc/archive/2001/ 200104/bulletin.htm

Hubbard, L.L., 1987, Low streamflow conditions in the Western States during 1987: U.S. Geological Survey Water-Resources Investigations Report 87-4267, 29 p.

National Oceanic and Atmospheric Administration, National Climatic Data Center, Climate of 2001September: U.S. Regional Drought Watch, accessed May 2002, at URL

http://www.ncdc.noaa.gov/oa/climate/research/ 2001/sep/drought-regional-overview.html

-National Weather Service, Climate Prediction Center, 2001 Drought severity index by division: accessed December 2001, at URL http://www.cpc.ncep.noaa.gov/products/ analysis_monitoring/regional_monitoring/ palmer/2001/weekly_PALMER_2001.html

_U.S. Drought Monitor, September 2001: accessed May 2002, at URL http://www.drought.unl.edu/dm/archive/2001/ drmon0925.htm 
Natural Resources Conservation Service, National Water and Climate Center, Precipitation reports, water years 2000 and 2001: accessed May 2002, at URL

http://www.wcc.nrcs.usda.gov/water/precip/precip.html Reservoir reports, water year 2001: accessed May 2002, at URL

http://www.wcc.nrcs.usda.gov/water/reservoir/ resv_rpt.html
- Snowpack reports, water years 2000 and 2001: accessed May 2002, at URL

http://www.wcc.nrcs.usda.gov/water/snow/ snow_rpt.html

Paulson, R.W., Chase, E.B., Roberts, R.S., and Moody, D.W., 1991, National water summary 1988-89hydrologic events and floods and droughts: U.S. Geological Survey Water-Supply Paper 2375, $591 \mathrm{p}$. 
Figures 1-11 


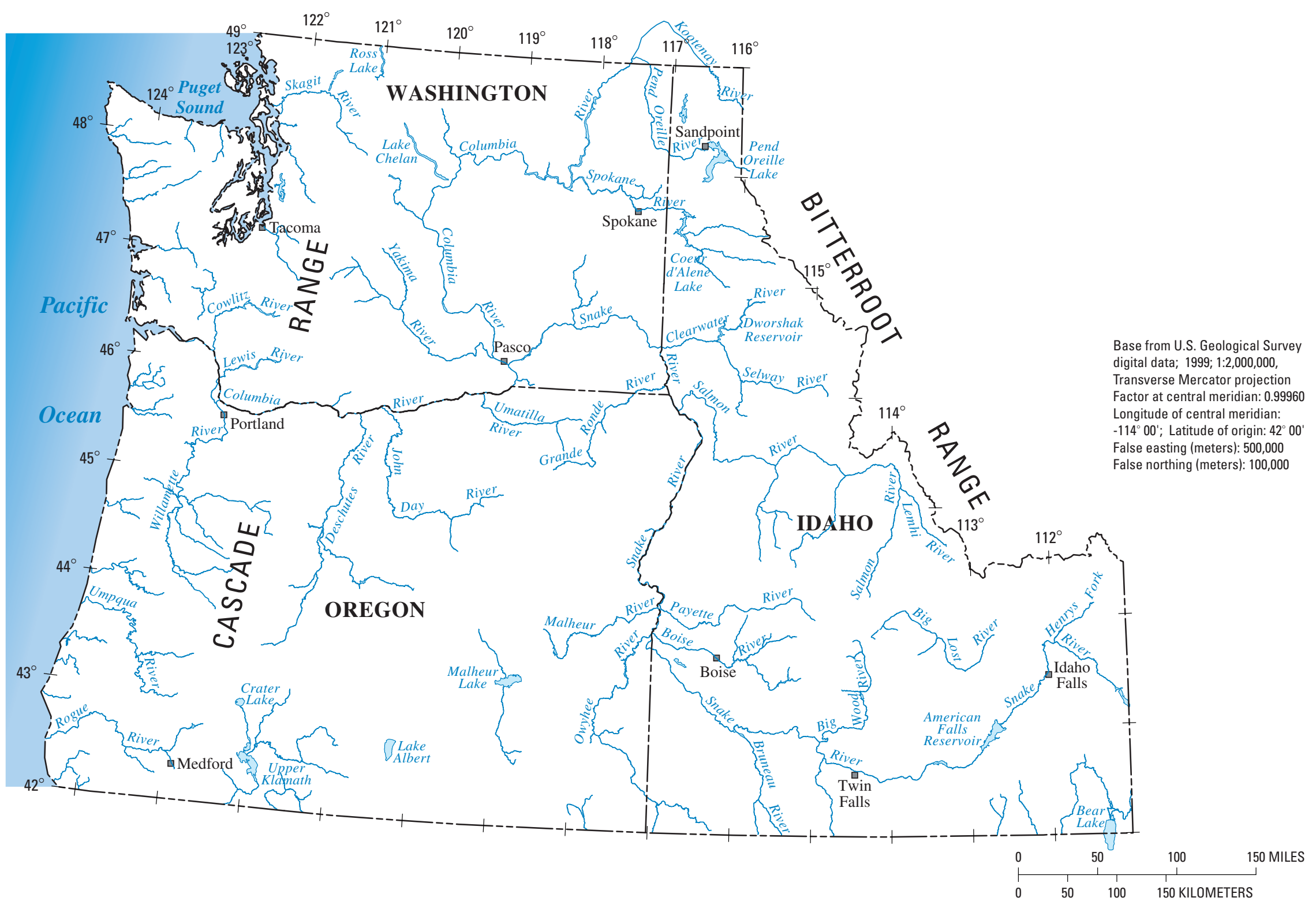

Figure 1. Location of study area, major lakes, reservoirs, and rivers, Washington, Oregon, and Idaho. 


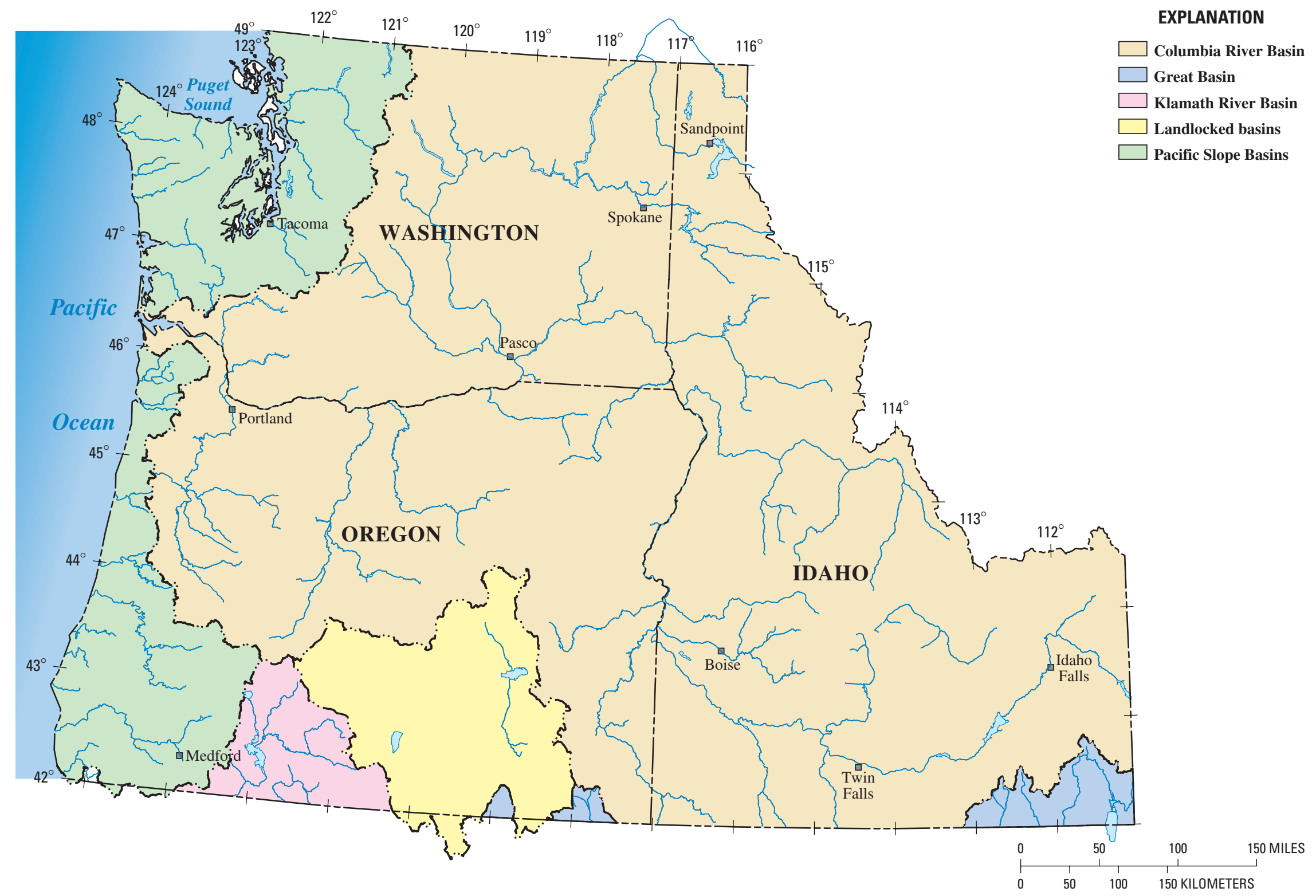

Figure 2. Location of major basins, Washington, Oregon, and Idaho. 


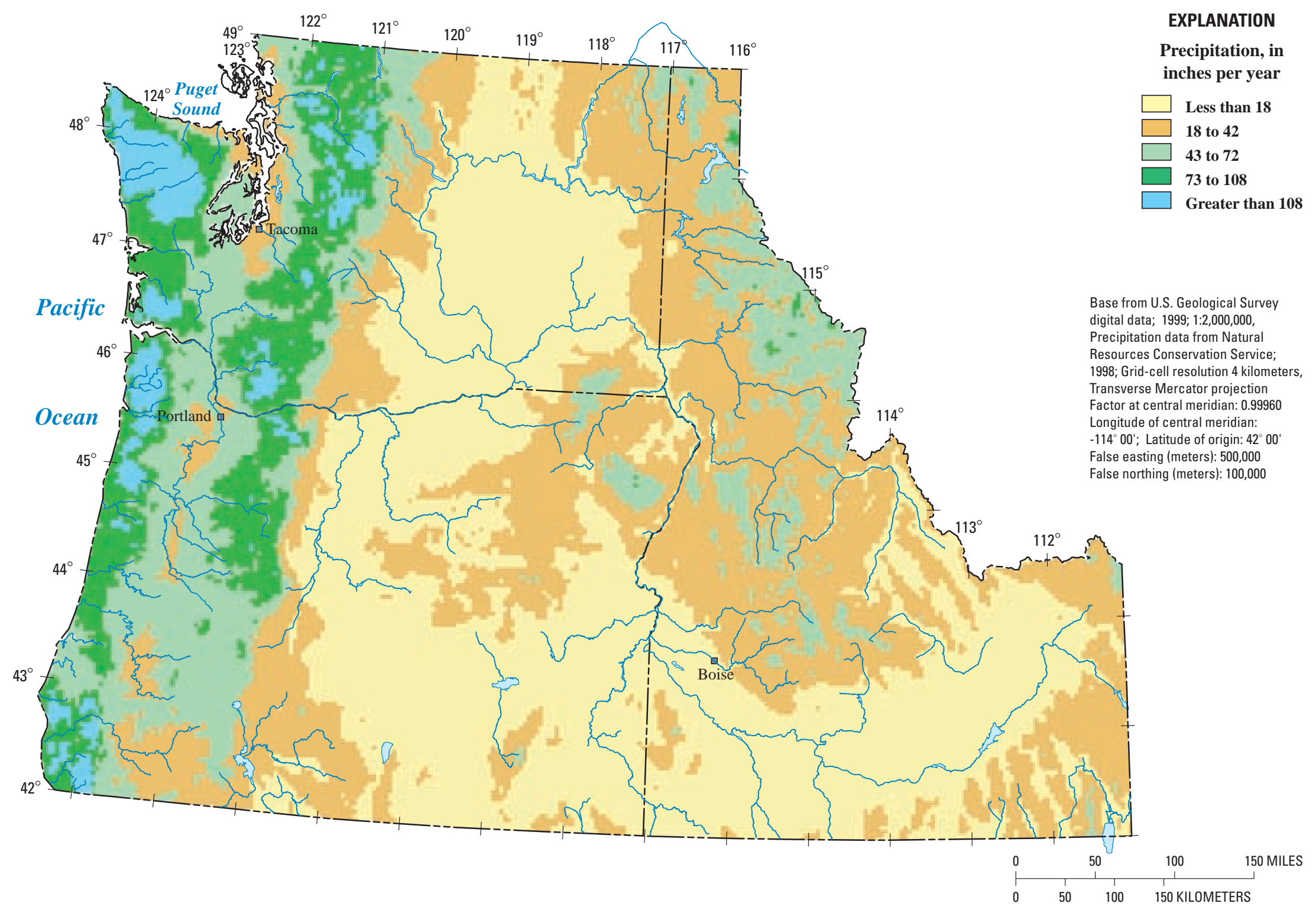



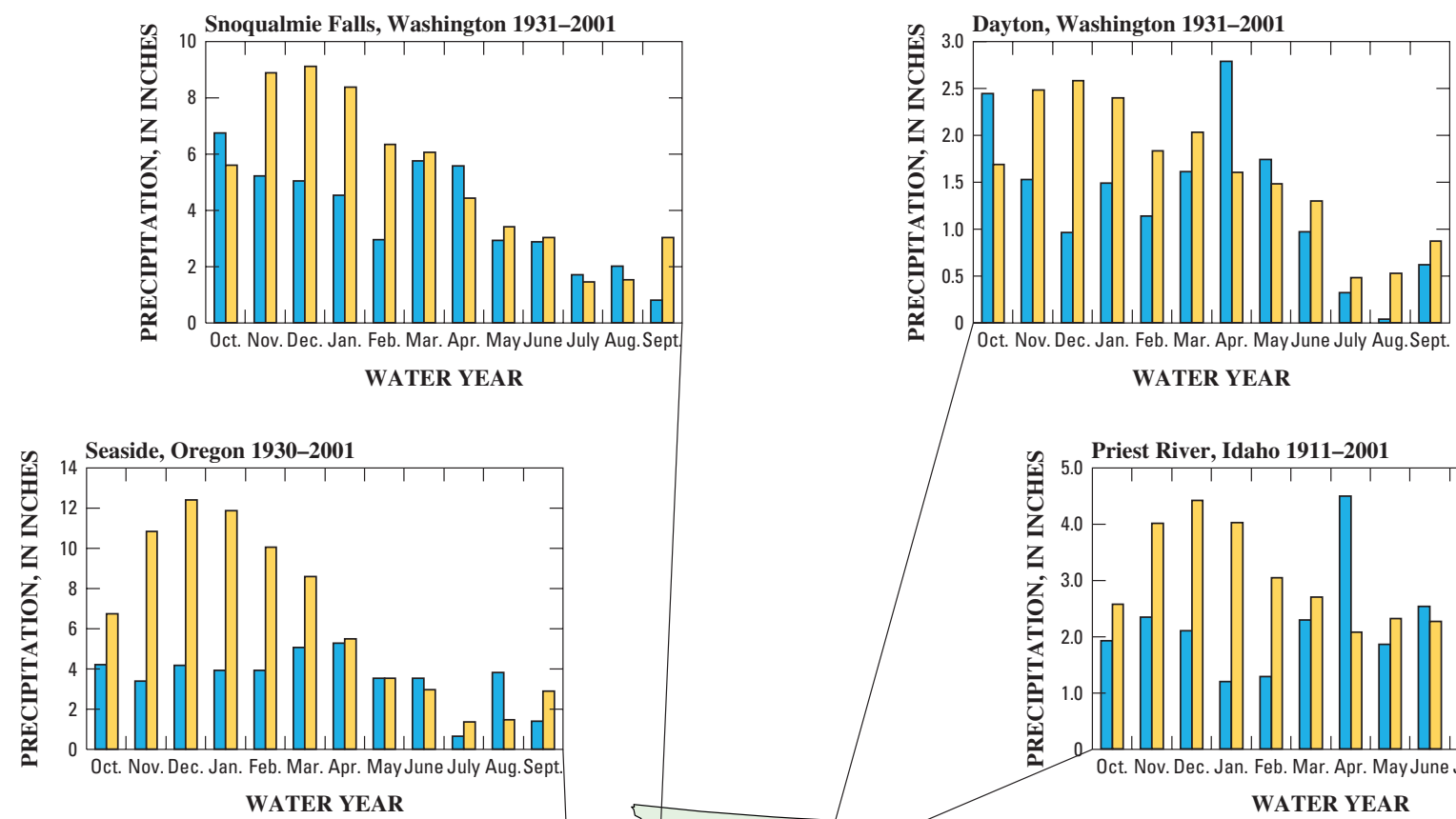

WATER YEAR

EXPLANATION

Water year 2001

$\square$ Normal (1961-90)
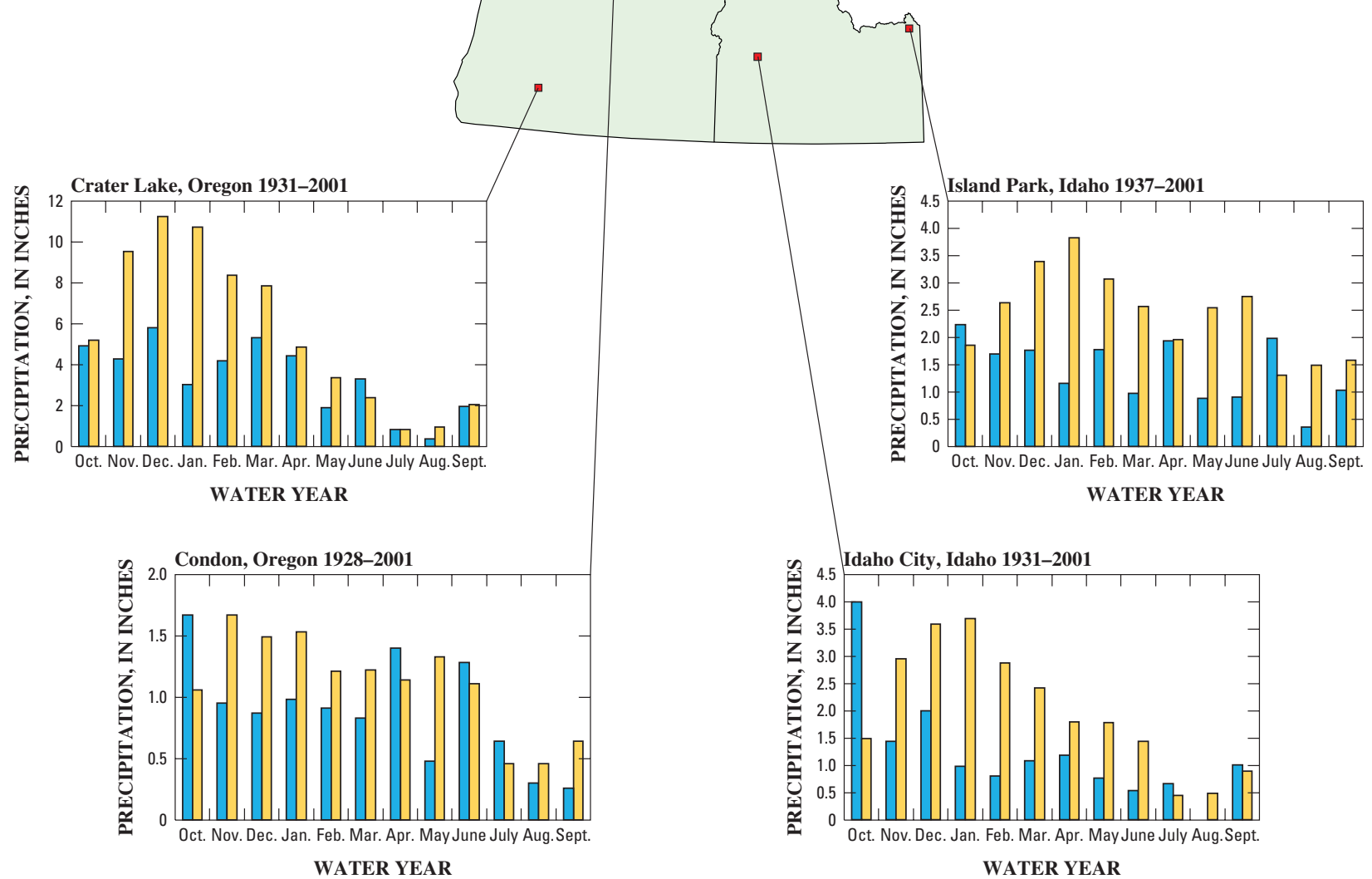

WATER YEAR

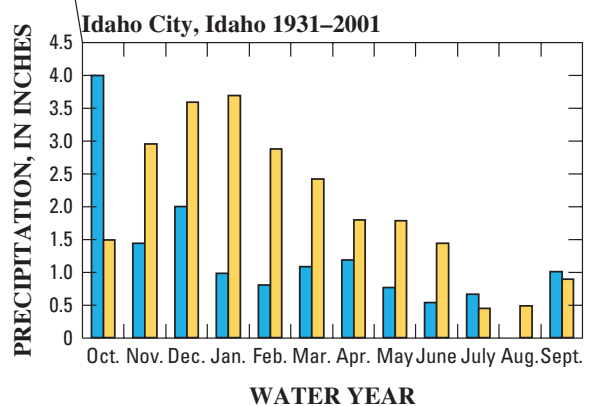

Figure 4. Monthly precipitation during water year 2001, and monthly normal precipitation during 1961-90 at selected weather stations in Washington, Oregon, and Idaho. (Natural Resources Conservation Service, Precipitation Reports, 2002) 


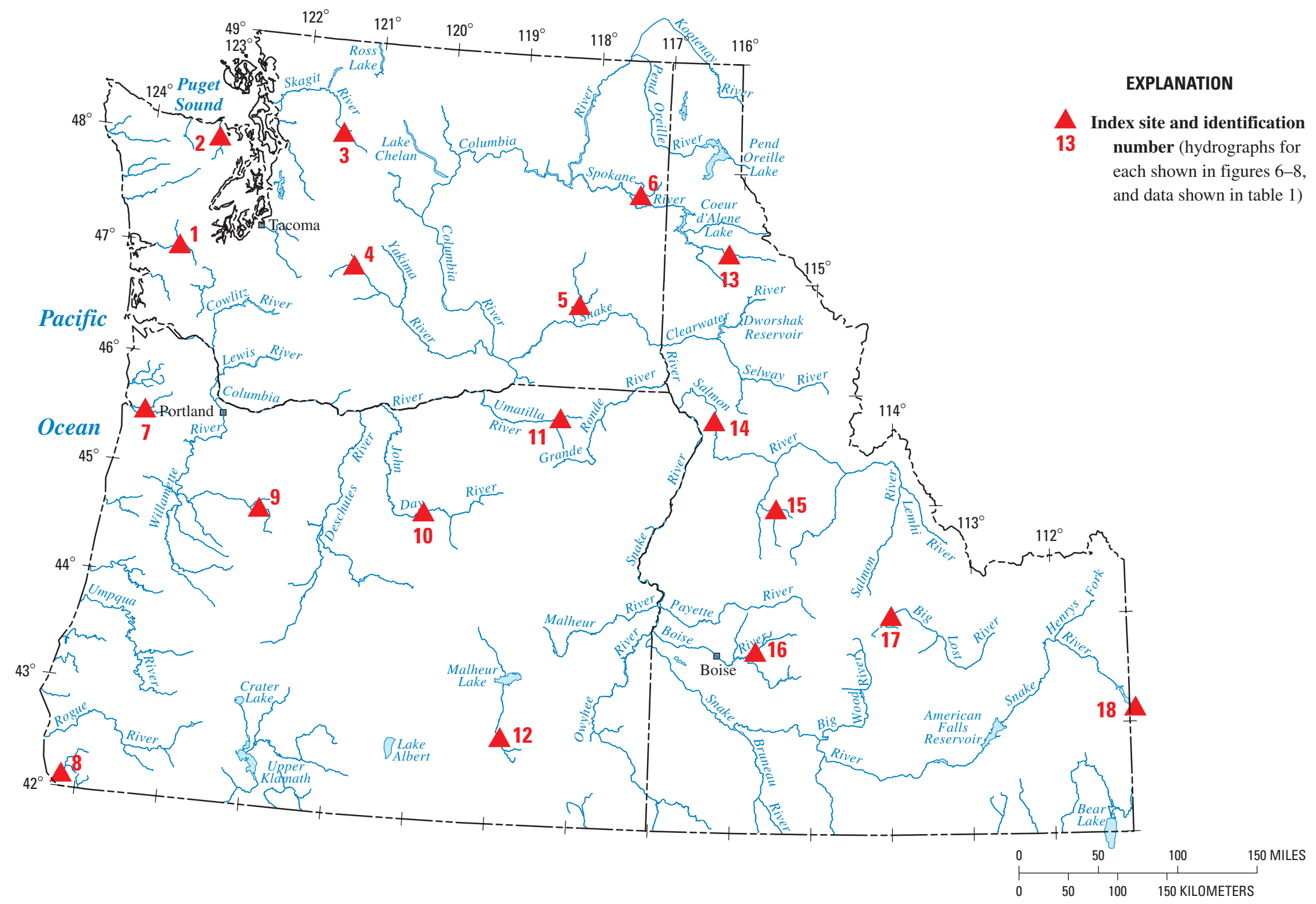

Figure 5. Location of long-term gaging stations used as index sites, Washington, Oregon, and Idaho. 

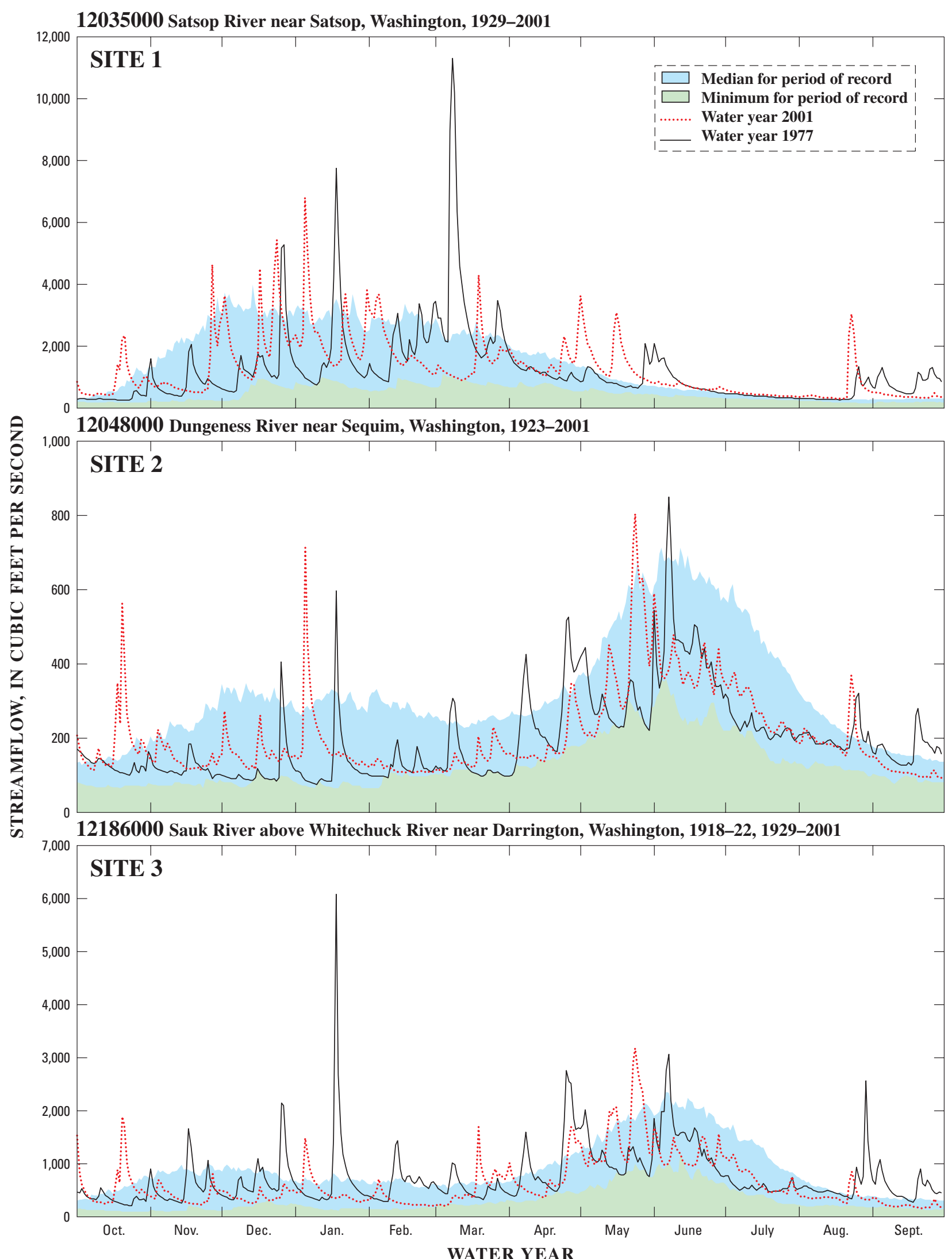

Figure 6. Daily mean streamflow for water years 1977 and 2001, and long-term minimum and median streamflow for selected gaging stations in Washington. (Locations shown in figure 5) 
12488500 American River near Nile, Washington, 1940-2001
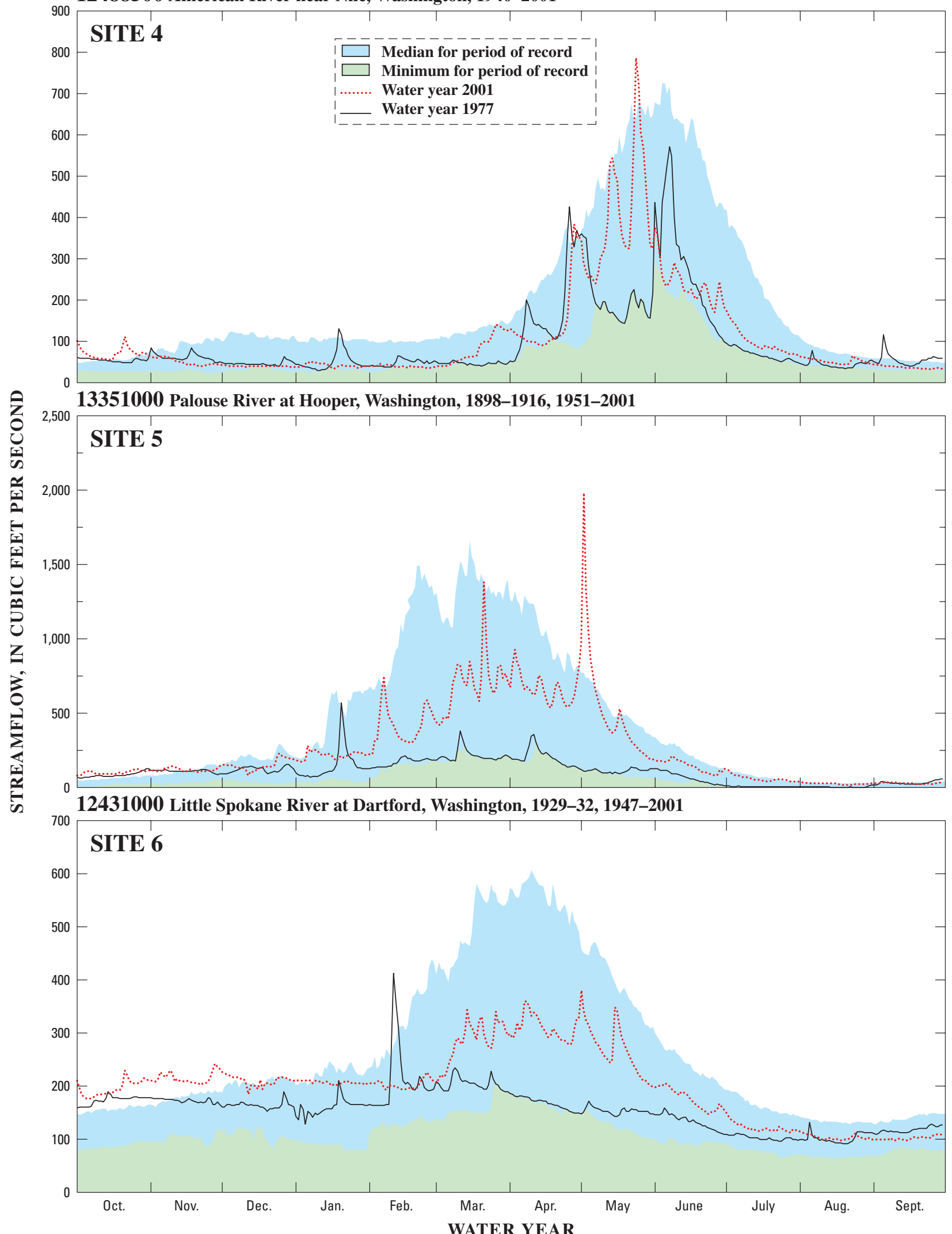

Figure 6. Daily mean streamflow for water years 1977 and 2001, and long-term minimum and median streamflow for selected gaging stations in Washington-Continued. (Locations shown in figure 5) 
14301500 Wilson River near Tillamook, Oregon, 1915-16, 1931-2001
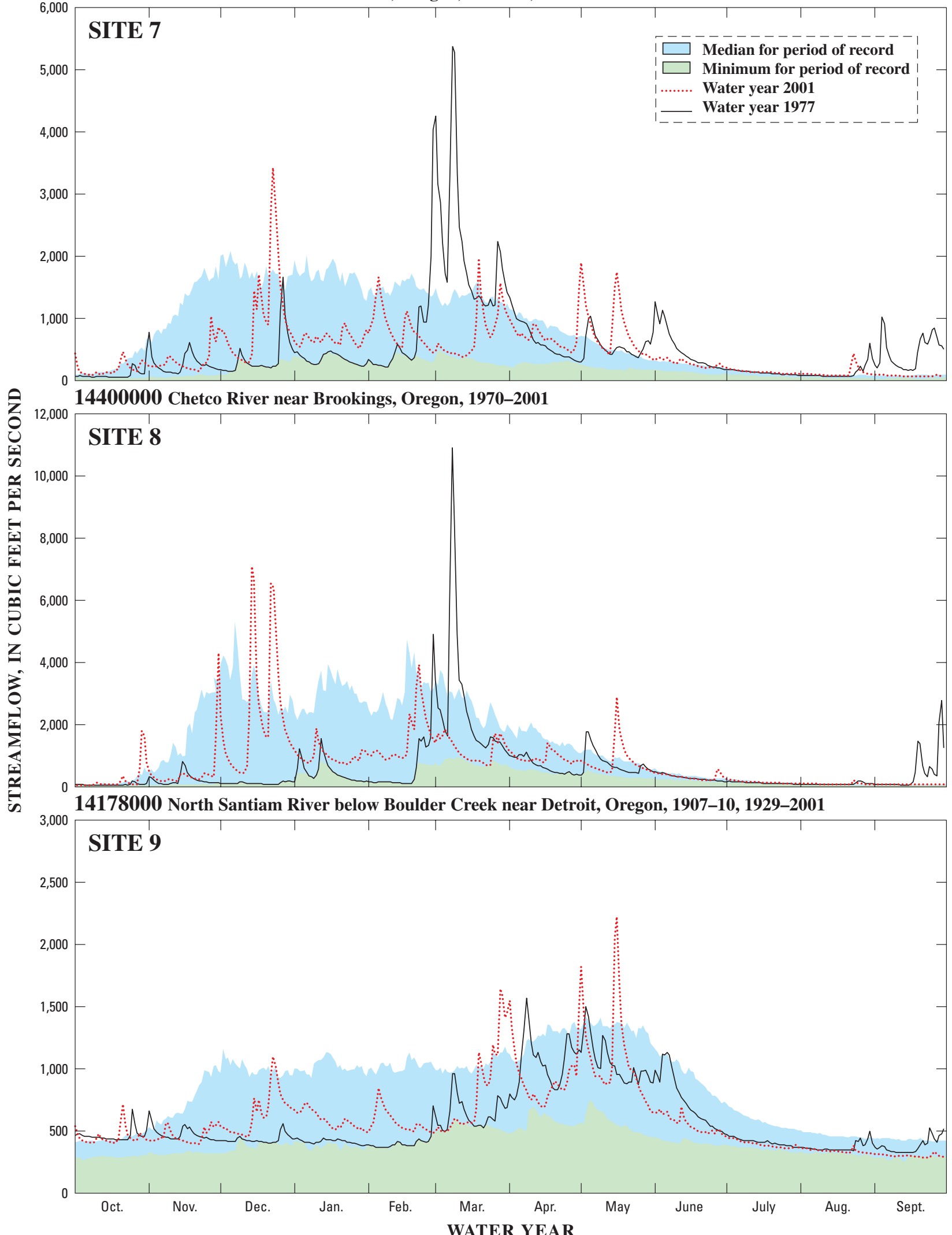

Figure 7. Daily mean streamflow for water years 1977 and 2001, and long-term minimum and median streamflow for selected gaging stations in Oregon. (Locations shown in figure 5) 
14046500 John Day River at Service Creek, Oregon, 1930-2001
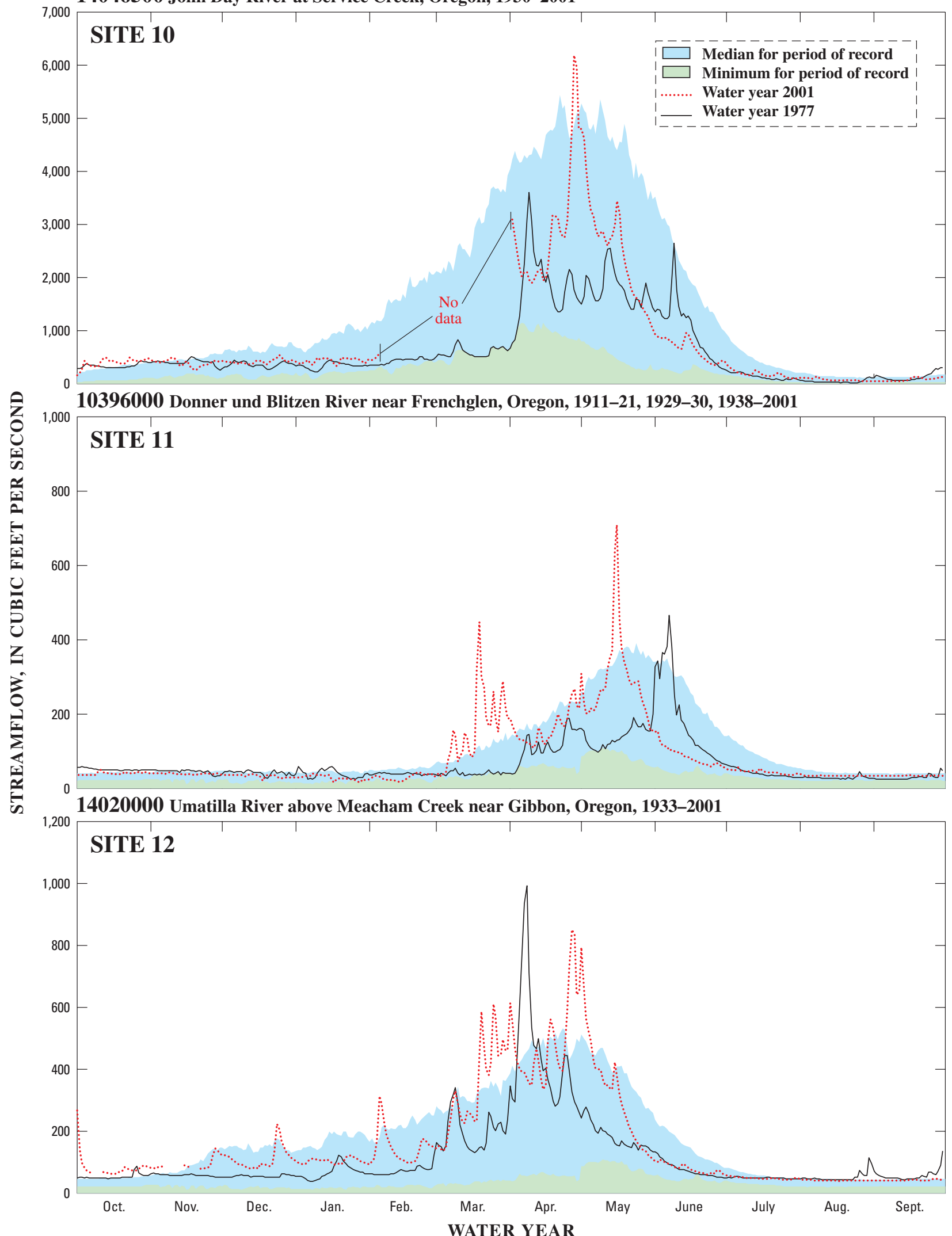

Figure 7. Daily mean streamflow for water years 1977 and 2001, and long-term minimum and median streamflow for selected gaging stations in Oregon-Continued. (Locations shown in figure 5) 
12414500 St. Joe River at Calder, Idaho, 1911-12, 1920-2001
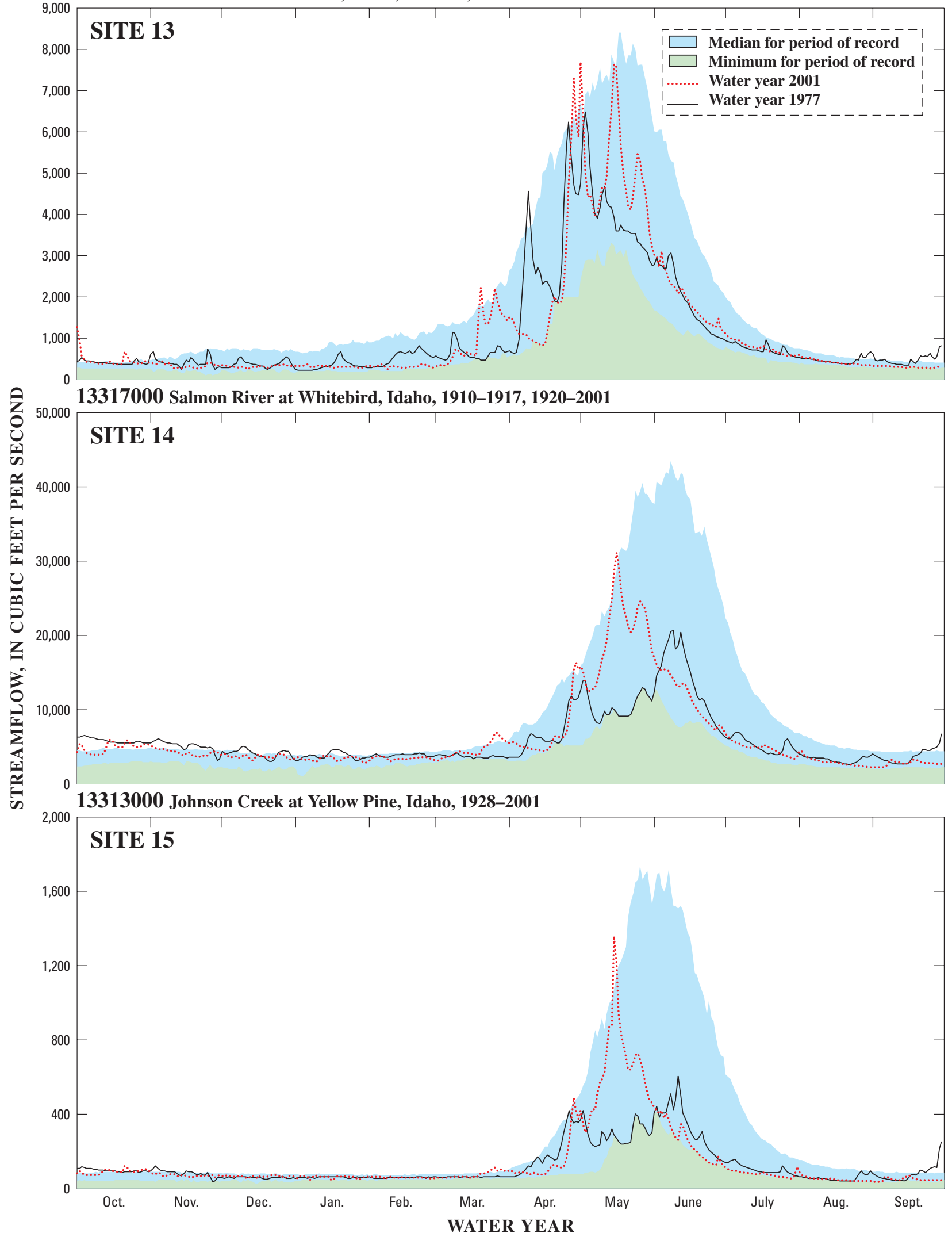

Figure 8. Daily mean streamflow for water years 1977 and 2001, and long-term minimum and median streamflow for selected gaging stations in Idaho. (Locations shown in figure 5) 
13185000 Boise River near Twin Springs, Idaho, 1911-2001
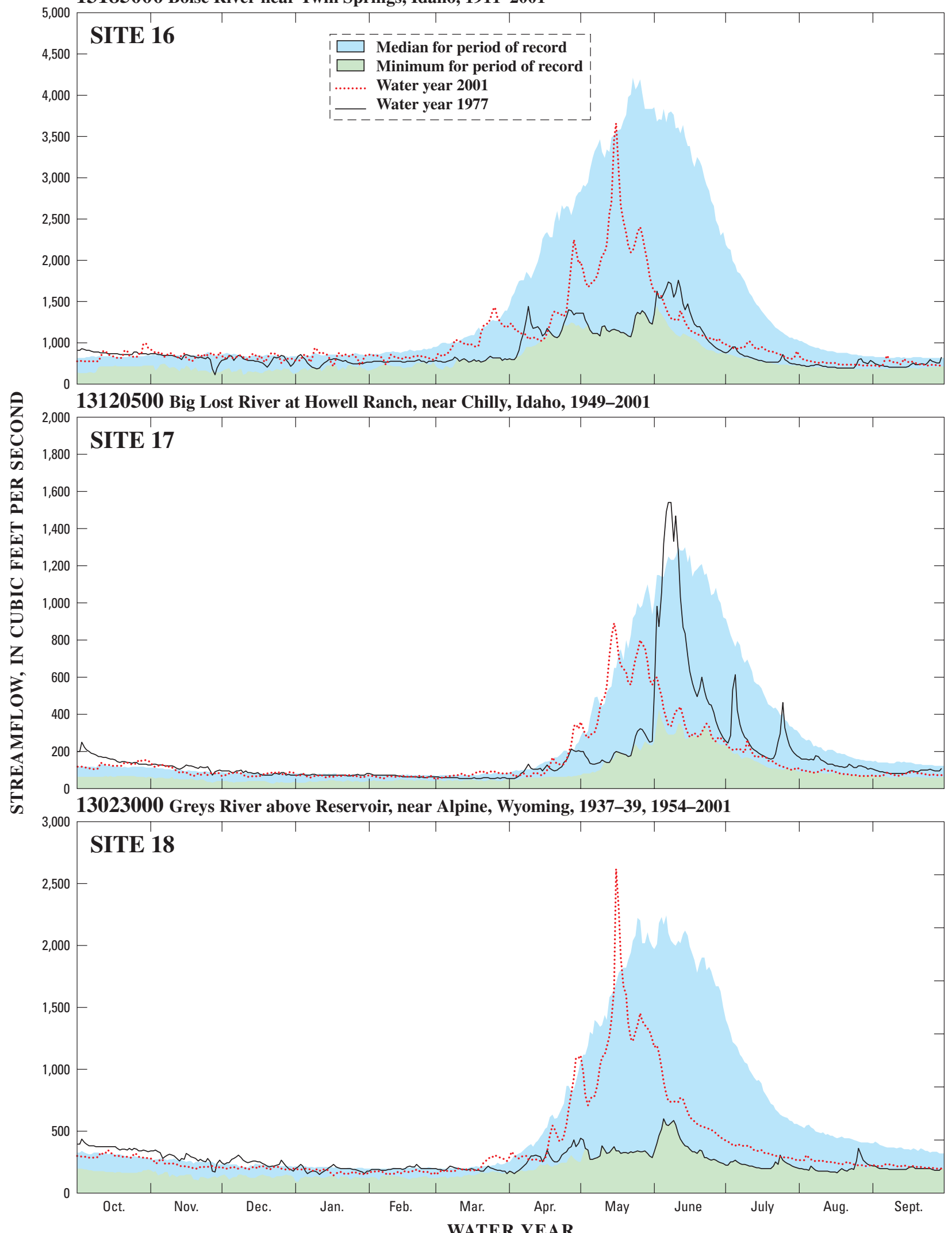

Figure 8. Daily mean streamflow for water years 1977 and 2001, and long-term minimum and median streamflow for selected gaging stations in Idaho-Continued. (Locations shown in figure 5) 


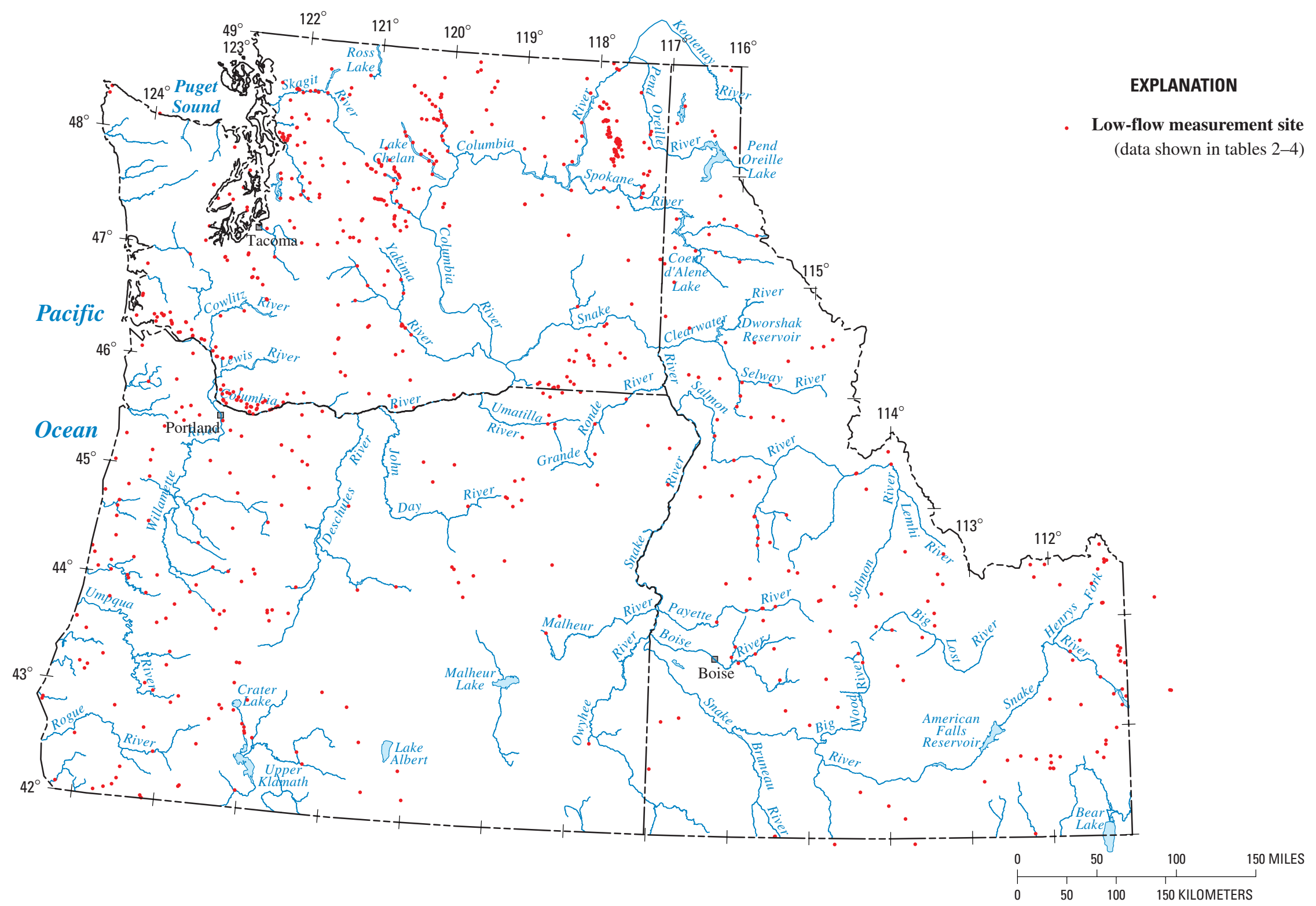

Figure 9. Location of low-flow measurement sites, Washington, Oregon, and Idaho, water year 2001. 


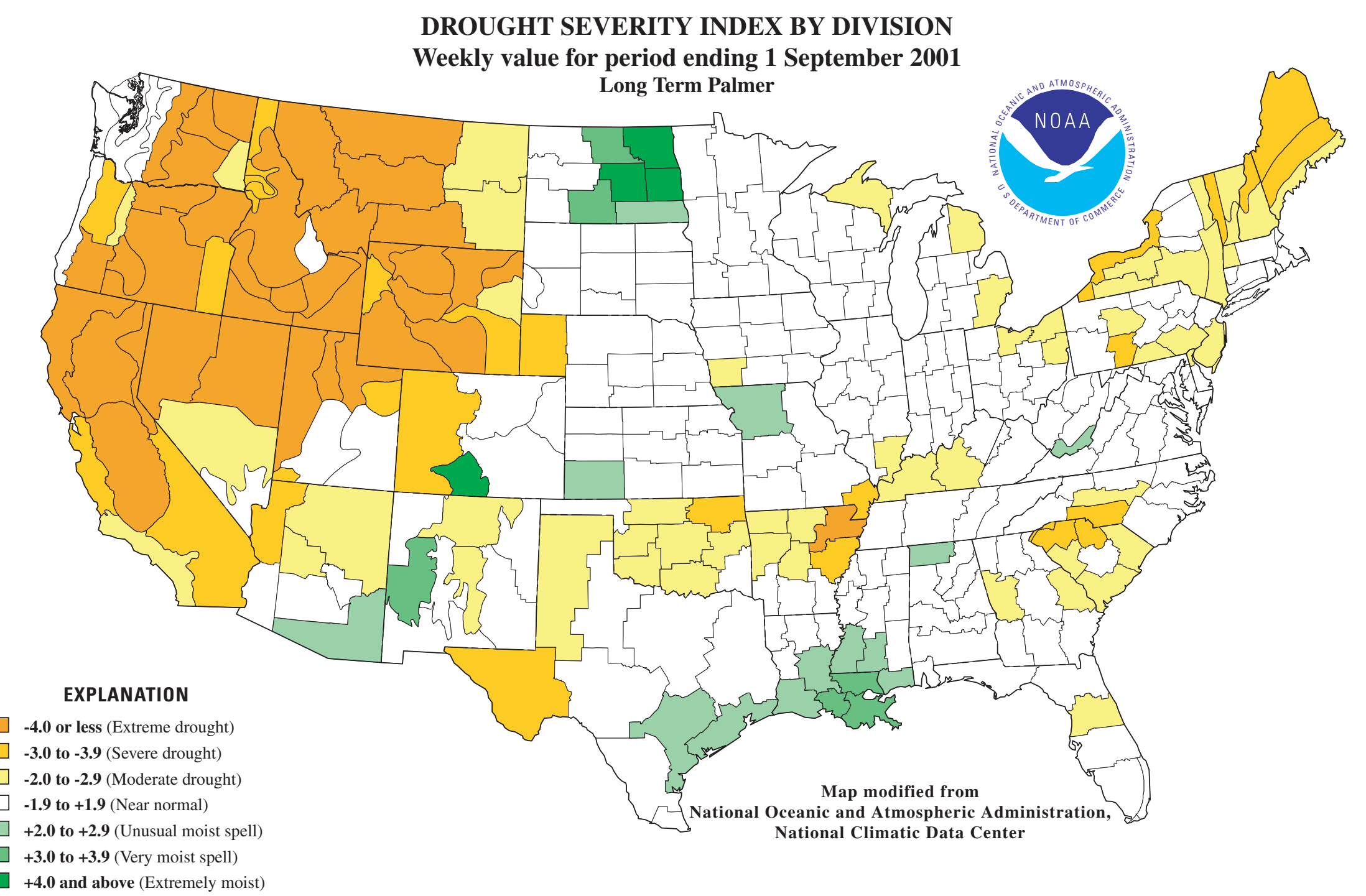

Figure 10. Palmer drought severity index map for the continental United States, September 1, 2001. 


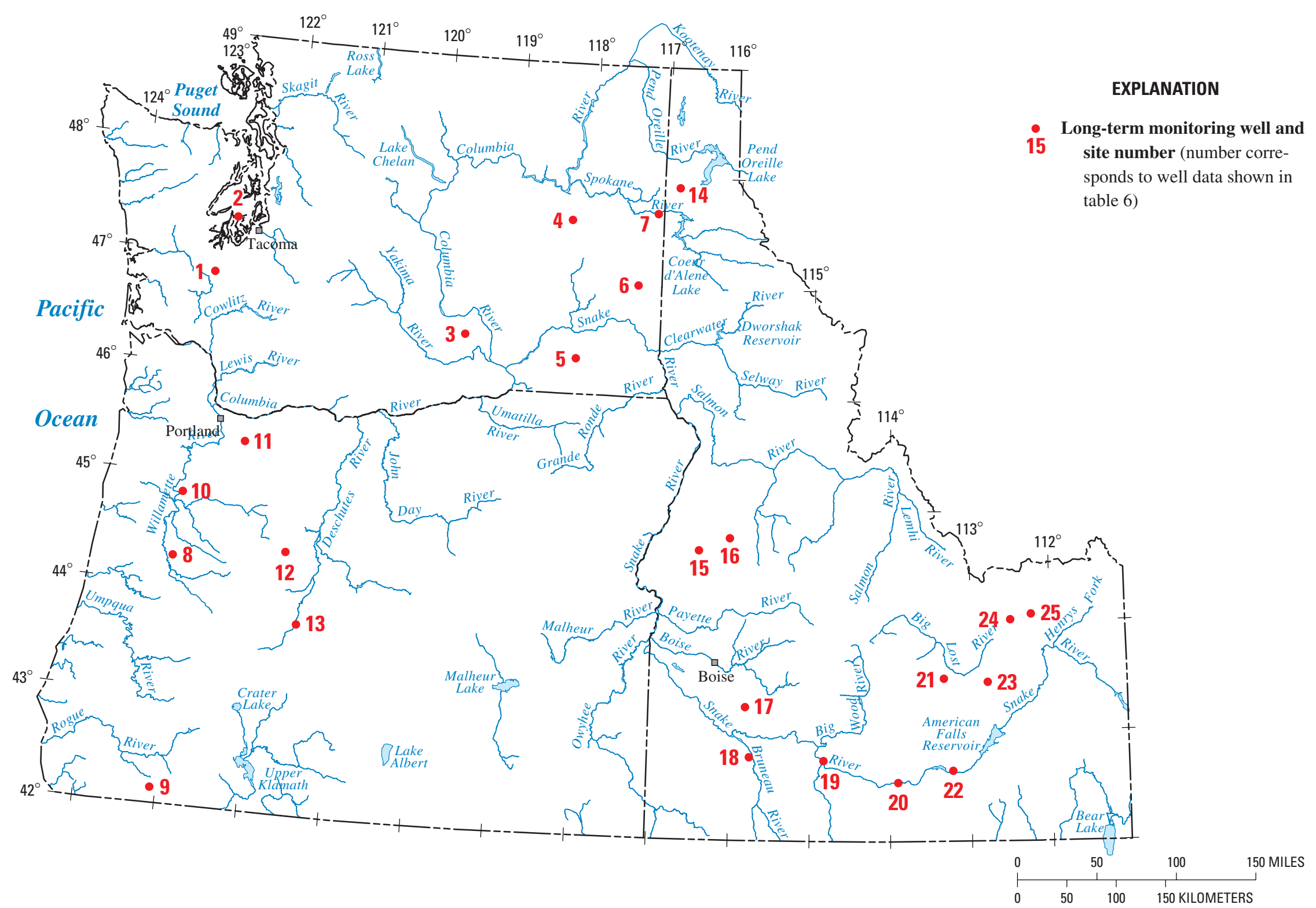

Figure 11. Location of long-term monitoring wells, Washington, Oregon, and Idaho, water year 2002. 
Table 2 
Table 2. Low-flow measurement data for 410 selected sites in Washington, water years 1977 and 2001

[Latitude and longitude are based on the North American Datum of 1983 and shown in degrees, minutes, seconds; $\mathrm{ft}^{3} / \mathrm{s}$, cubic feet per second; WA, Washington; ---, no number assigned]

\begin{tabular}{|c|c|c|c|c|c|c|c|}
\hline \multirow{2}{*}{$\begin{array}{l}\text { Gaging- } \\
\text { station } \\
\text { number }\end{array}$} & \multirow[b]{2}{*}{ Gaging-station name } & \multirow[b]{2}{*}{ Latitude } & \multirow[b]{2}{*}{ Longitude } & \multicolumn{2}{|c|}{ Water year 2001} & \multicolumn{2}{|c|}{ Water year 1977} \\
\hline & & & & Date & $\begin{array}{c}\text { Discharge, } \\
\text { in } \mathrm{ft}^{3} / \mathrm{s}\end{array}$ & Date & $\begin{array}{c}\text { Discharge, } \\
\text { in } \mathrm{ft}^{3} / \mathrm{s}\end{array}$ \\
\hline \multicolumn{8}{|c|}{ Willapa Bay Basin } \\
\hline 12009500 & Bear Branch near Naselle, WA & $46^{\circ} 19^{\prime} 47^{\prime \prime}$ & $123^{\circ} 54^{\prime} 40^{\prime \prime}$ & $9-20-2001$ & 11.0 & & \\
\hline 12011103 & North Nemah River near Nemah, WA & $46^{\circ} 30^{\prime} 49^{\prime \prime}$ & $123^{\circ} 51^{\prime} 46^{\prime \prime}$ & $9-21-2001$ & 16.0 & & \\
\hline 12011200 & Williams Creek near South Bend, WA & $46^{\circ} 31^{\prime} 49^{\prime \prime}$ & $123^{\circ} 51^{\prime} 39^{\prime \prime}$ & $9-21-2001$ & 13.0 & & \\
\hline 12017000 & North River near Raymond, WA & $46^{\circ} 48^{\prime} 26^{\prime \prime}$ & $123^{\circ} 51^{\prime} 02^{\prime \prime}$ & $9-21-2001$ & 79.0 & $10-12-1977$ & 245 \\
\hline \multicolumn{8}{|c|}{ Chehalis River Basin } \\
\hline--- & Porter Creek at Railroad Bridge at Porter, WA & $46^{\circ} 56^{\prime} 14^{\prime \prime}$ & $123^{\circ} 18^{\prime} 34^{\prime \prime}$ & $9-20-2001$ & 15.0 & & \\
\hline \multicolumn{8}{|c|}{ Between Calawah River Basin and Cape Flattery } \\
\hline 12043163 & Sooes River below Miller Creek near Ozette, WA & $48^{\circ} 17^{\prime} 43^{\prime \prime}$ & $124^{\circ} 39^{\prime} 11^{\prime \prime}$ & $9-13-2001$ & 41.0 & & \\
\hline 12043173 & Wasatch River below Educket Creek at Neah Bay, WA & $48^{\circ} 21^{\prime} 24^{\prime \prime}$ & $124^{\circ} 38^{\prime} 08^{\prime \prime}$ & $9-13-2001$ & 7.6 & & \\
\hline \multicolumn{8}{|c|}{ Between Cape Flattery and Elwha River Basin } \\
\hline 12043430 & East Twin River near Pysht, WA & $48^{\circ} 09^{\prime} 47^{\prime \prime}$ & $123^{\circ} 56^{\prime} 43^{\prime \prime}$ & $9-14-2001$ & 4.0 & & \\
\hline \multicolumn{8}{|c|}{ Between Dungeness and Quilcene River Basins } \\
\hline 12050500 & Snow Creek near Maynard, WA & $47^{\circ} 56^{\prime} 36^{\prime \prime}$ & $122^{\circ} 53^{\prime} 12^{\prime \prime}$ & $9-14-2001$ & 2.1 & & \\
\hline \multicolumn{8}{|c|}{ Between Duckabush and Skokomish River Basins } \\
\hline \multirow[t]{2}{*}{12054600} & Jefferson Creek near Eldon, WA & $47^{\circ} 35^{\prime} 00^{\prime \prime}$ & $123^{\circ} 06^{\prime} 27^{\prime \prime}$ & $9-18-2001$ & 20.0 & & \\
\hline & Hamma Hamma River near Eldon, WA & $47^{\circ} 35^{\prime} 15^{\prime \prime}$ & $123^{\circ} 06^{\prime} 18^{\prime \prime}$ & $9-14-2001$ & 71.0 & & \\
\hline \multicolumn{8}{|c|}{ Between Skokomish and Deschutes River Basins } \\
\hline 12063500 & Union River near Belfair, WA & $47^{\circ} 28^{\prime} 19^{\prime \prime}$ & $122^{\circ} 49^{\prime} 44^{\prime \prime}$ & $9-21-2001$ & 14.0 & & \\
\hline 12067500 & Tahuya River near Belfair, WA & $47^{\circ} 30^{\prime} 59^{\prime \prime}$ & $122^{\circ} 53^{\prime} 04^{\prime \prime}$ & $9-21-2001$ & .30 & & \\
\hline 12068500 & Dewatto River near Dewatto, WA & $47^{\circ} 28^{\prime} 05^{\prime \prime}$ & $123^{\circ} 01^{\prime} 40^{\prime \prime}$ & $9-18-2001$ & 11.0 & & \\
\hline 12070000 & Dogfish Creek near Poulsbo, WA & $47^{\circ} 45^{\prime} 10^{\prime \prime}$ & $122^{\circ} 38^{\prime} 40^{\prime \prime}$ & $9-20-2001$ & 4.7 & & \\
\hline 12072000 & Chico Creek near Bremerton, WA & $47^{\circ} 35^{\prime} 27^{\prime \prime}$ & $122^{\circ} 42^{\prime} 33^{\prime \prime}$ & $9-20-2001$ & 1.8 & & \\
\hline 12072800 & Purdy Creek at Purdy,WA & $47^{\circ} 23^{\prime} 17^{\prime \prime}$ & $122^{\circ} 37^{\prime} 34^{\prime \prime}$ & $9-20-2001$ & 1.7 & & \\
\hline 12073000 & Burley Creek at Burley, WA & $47^{\circ} 24^{\prime} 54^{\prime \prime}$ & $122^{\circ} 37^{\prime} 54^{\prime \prime}$ & $9-20-2001$ & 14.0 & & \\
\hline 12077000 & Goldsborough Creek at Shelton, WA & $47^{\circ} 12^{\prime} 29^{\prime \prime}$ & $123^{\circ} 06^{\prime} 04^{\prime \prime}$ & $9-21-2001$ & 29.0 & & \\
\hline 12078400 & Kennedy Creek near Kamilche, WA & $47^{\circ} 04^{\prime} 38^{\prime \prime}$ & $123^{\circ} 07^{\prime} 39^{\prime \prime}$ & $9-21-2001$ & 3.2 & & \\
\hline \multicolumn{8}{|c|}{ Deschutes River Basin } \\
\hline--- & Little Deschutes River near La Grande, WA & $46^{\circ} 47^{\prime} 30^{\prime \prime}$ & $122^{\circ} 23^{\prime} 23^{\prime \prime}$ & $9-13-2001$ & .90 & & \\
\hline--- & Deschutes River near La Grande, WA & $46^{\circ} 47^{\prime} 30^{\prime \prime}$ & $122^{\circ} 23^{\prime} 33^{\prime \prime}$ & $9-13-2001$ & 15.0 & & \\
\hline 12078850 & Deschutes River above Mitchell Creek near La Grande, WA & $46^{\circ} 47^{\prime} 43^{\prime \prime}$ & $122^{\circ} 27^{\prime} 26^{\prime \prime}$ & $9-13-2001$ & 12.0 & $9-12-1977$ & 22.2 \\
\hline 12078900 & Mitchell Creek near La Grande, WA & $46^{\circ} 47^{\prime} 25^{\prime \prime}$ & $122^{\circ} 28^{\prime} 04^{\prime \prime}$ & $9-13-2001$ & 2.3 & $9-12-1977$ & 2.7 \\
\hline 12079382 & Offutt Lake Outlet near Olympia, WA & $46^{\circ} 55^{\prime} 12^{\prime \prime}$ & $122^{\circ} 48^{\prime} 50^{\prime \prime}$ & $9-12-2001$ & 0 & & \\
\hline 12079400 & Deschutes River near Turnwater, WA & $46^{\circ} 56^{\prime} 42^{\prime \prime}$ & $122^{\circ} 50^{\prime} 59^{\prime \prime}$ & $9-12-2001$ & 45.0 & $9-13-1977$ & 58.7 \\
\hline 12079500 & Spurgeon Creek near Olympia, WA & $46^{\circ} 56^{\prime} 57^{\prime \prime}$ & $122^{\circ} 50^{\prime} 39^{\prime \prime}$ & $9-12-2001$ & 3.7 & $9-13-1977$ & 5.4 \\
\hline \multicolumn{8}{|c|}{ Nisqually River Basin } \\
\hline--- & Graiville Creek near Roy, WA & $46^{\circ} 59^{\prime} 28^{\prime \prime}$ & $122^{\circ} 32^{\prime} 50^{\prime \prime}$ & $9-20-2001$ & 0 & & \\
\hline--- & Little Nisqually River near Alder, WA & $46^{\circ} 44^{\prime} 08^{\prime \prime}$ & $122^{\circ} 18^{\prime} 52^{\prime \prime}$ & $9-20-2001$ & 11.0 & & \\
\hline 12089000 & Tanwax Creek near McKenna, WA & $46^{\circ} 51^{\prime} 59^{\prime \prime}$ & $122^{\circ} 27^{\prime} 04^{\prime \prime}$ & $9-20-2001$ & 0 & & \\
\hline 12090200 & Muck Creek at Roy, WA & $47^{\circ} 00^{\prime} 19^{\prime \prime}$ & $122^{\circ} 32^{\prime} 36^{\prime \prime}$ & $9-20-2001$ & 0 & & \\
\hline
\end{tabular}


Table 2. Low-flow measurement data for 410 selected sites in Washington, water years 1977 and $2001--$ Continued

\begin{aligned} & $\begin{array}{r}\text { Gaging- } \\ \text { station } \\ \text { number }\end{array} \\ &$\hline 12093000 Kapowsin Creek near Kapowsin, WA \\ & 12097000 White River at Greenwater, WA \\ & 12097850 White River below Clearwater River near Buckley, WA \\ & \\ & 12103020 Hylebos Creek at Highway 99 at Fife, WA \\ & \\ & 12103380 Green River above Twin Camp Creek near Lester, WA \\ & 12107300 Icy Creek near Black Diamond, WA \\ & 12111500 Covington Creek near Black Diamond, WA \\ & \\ & 12119600 May Creek at mouth, near Renton, WA \\ & 12120500 Juanita Creek near Kirkland, WA \\ & 12121500 East Fork Issaquah Creek at Issaquah, WA \\ & 12121700 Tibbetts Creek near Issaquah, WA \\ & 12122500 Bear Creek near Redmond, WA \\ & 12127100 Swamp Creek at Kenmore, WA \\ & \\ & 12131000 Beckler River near Skykomish, WA \\ & 12133500 Troublesome Creek near Index, WA \\ & 12135000 Wallace River at Gold Bar, WA \\ & 12136000 Olney Creek near Startup, WA \\ & 12141000 Woods Creek near Monroe, WA \\ & 12142200 Calligan Creek near Snoqualmie, WA \\ & 12142300 Hancock Creek near Snoqualmie, WA \\ & 12145000 Tokul Creek near Snoqualmie, WA \\ & 12146000 Patterson Creek near Fall City, WA \\ & 12147000 Griffin Creek near Carnation, WA \\ & 12148700 Stossel Creek near Carnation, WA \\ & 12150500 Cherry Creek near Duvall, WA \\ & 12152500 Pilchuck River near Granite Falls, WA \\ & 12153000 Little Pilchuck Creek near Lake Stevens, WA \\ & \\ & 12157000 Unnamed Spring \#1 near Tulalip, WA \\ & 12157020 West Fork Quilceda Creek near Marysville, WA \\ & 12157170 Quilceda Creek Tributary near Marysville,WA \\ & \hline Sturgeon Creek at Marysville,WA \\ & Mission Creek near Marysville,WA \\ & \\ & $121570 e k$ Tributary \#1 near Tulalip,WA \end{aligned}

en

(n)

Water year 2001

WA

$47^{\circ} 08^{\prime} 48^{\prime \prime} 121^{\circ} 51^{\prime} 36^{\prime \prime}$
Between Puyallup and Duwamish River Basins

Longitude Date in $\mathrm{ft}^{3} / \mathrm{s}$

$\begin{array}{cccc}\text { Puyallup River Basin } & & & \\ 46^{\circ} 59^{\prime} 44^{\prime \prime} & 122^{\circ} 11^{\prime} 49^{\prime \prime} & 9-20-2001 & 4.3 \\ 47^{\circ} 08^{\prime} 48^{\prime \prime} & 121^{\circ} 38^{\prime} 54^{\prime \prime} & 9-19-2001 & 378\end{array}$

$\begin{array}{lll} & 9-19-2001 & 378 \\ & 9-19-2001 & 499\end{array}$

Duwamish River Basin

$47^{\circ} 10^{\prime} 54^{\prime \prime} \quad 121^{\circ} 23^{\prime} 19^{\prime \prime} \quad 9-18-2001 \quad 5.2$

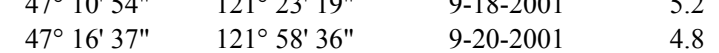

$47^{\circ} 20^{\prime} 08^{\prime \prime} \quad 122^{\circ} 02^{\prime} 47^{\prime \prime} \quad 9-20-2001$

Lake Washington Basin

$47^{\circ} 31^{\prime} 47^{\prime \prime} \quad 122^{\circ} 12^{\prime} 04^{\prime \prime} \quad 9-18-2001 \quad 3.7$

$47^{\circ} 42^{\prime} 19^{\prime \prime} \quad 122^{\circ} 13^{\prime} 00^{\prime \prime} \quad 9-18-2001 \quad 2.7$

$47^{\circ} 32^{\prime} 07^{\prime \prime} \quad 122^{\circ} 02^{\prime} 15^{\prime \prime} \quad 9-19-2001$

$\begin{array}{llll}47^{\circ} 32^{\prime} 29^{\prime \prime} & 122^{\circ} 03^{\prime} 51^{\prime \prime} & 9-19-2001 & .34\end{array}$

$\begin{array}{llll}47^{\circ} 43^{\prime} 03^{\prime \prime} & 122^{\circ} 04^{\prime} 38^{\prime \prime} & 9-19-2001 & 8.1 \\ 47^{\circ} 45^{\prime} 21^{\prime \prime} & 122^{\circ} 14^{\prime} 01^{\prime \prime} & 9-18-2001 & 5.8\end{array}$

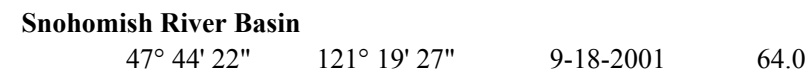

$\begin{array}{llll}47^{\circ} 44^{\prime} 22^{\prime \prime} & 121^{\circ} 19^{\prime} 27^{\prime \prime} & 9-18-2001 & 64.0 \\ 47^{\circ} 54^{\prime} 02^{\prime \prime} & 121^{\circ} 23^{\prime} 55^{\prime \prime} & 9-18-2001 & 42.0\end{array}$

$47^{\circ} 51^{\prime} 49^{\prime \prime} \quad 121^{\circ} 40^{\prime} 55^{\prime \prime} \quad 9-21-2001 \quad 18.0$

$47^{\circ} 55^{\prime} 39^{\prime \prime} \quad 121^{\circ} 43^{\prime} 07^{\prime \prime} \quad 9-18-2001 \quad 8.8$

$47^{\circ} 52^{\prime} 09^{\prime \prime} \quad 121^{\circ} 55^{\prime} 32^{\prime \prime} \quad 9-19-2001 \quad 19.0$

$47^{\circ} 36^{\prime} 06^{\prime \prime} \quad 121^{\circ} 41^{\prime} 10^{\prime \prime} \quad 9-17-2001 \quad .23$

$47^{\circ} 34^{\prime} 19^{\prime \prime} \quad 121^{\circ} 41^{\prime} 14^{\prime \prime} \quad 9-17-2001 \quad 4.4$

$47^{\circ} 33^{\prime} 13^{\prime \prime} \quad 121^{\circ} 50^{\prime} 09^{\prime \prime} \quad 9-20-2001 \quad 23.0$

$47^{\circ} 34^{\prime} 47^{\prime \prime} \quad 121^{\circ} 56^{\prime} 11^{\prime \prime} \quad 9-20-2001 \quad 7.3$

$\begin{array}{llll}47^{\circ} 36^{\prime} 57^{\prime \prime} & 121^{\circ} 54^{\prime} 13^{\prime \prime} & 9-20-2001 & 2.4 \\ 47^{\circ} 41^{\prime} 43^{\prime \prime} & 121^{\circ} 49^{\prime} 51^{\prime \prime} & 9-21-2001 & 1.2\end{array}$

$47^{\circ} 41^{\prime} 43^{\prime \prime} \quad 121^{\circ} 49^{\prime} 51^{\prime \prime} \quad 9-21-2001 \quad 1.2$

$47^{\circ} 44^{\prime} 37^{\prime \prime} \quad 121^{\circ} 56^{\prime} 29^{\prime \prime} \quad 9-20-2001 \quad 3.0$

$\begin{array}{lllr}48^{\circ} 03^{\prime} 16^{\prime \prime} & 121^{\circ} 57^{\prime} 25^{\prime \prime} & 9-19-2001 & 39.0 \\ 48^{\circ} 01^{\prime} 24^{\prime \prime} & 122^{\circ} 03^{\prime} 05^{\prime \prime} & 9-19-2001 & 1.7\end{array}$

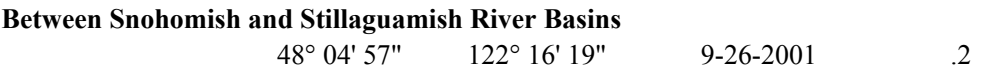

$\begin{array}{lllr}48^{\circ} 04^{\prime} 57^{\prime \prime} & 122^{\circ} 16^{\prime} 19^{\prime \prime} & 9-26-2001 & .2 \\ 48^{\circ} 03^{\prime} 26^{\prime \prime} & 122^{\circ} 14^{\prime} 23^{\prime \prime} & 9-26-2001 & .41\end{array}$

$48^{\circ} 06^{\prime} 17^{\prime \prime} \quad 122^{\circ} 09^{\prime} 46^{\prime \prime} \quad 9-19-2001 \quad 5.5$

$48^{\circ} 06^{\prime} 02^{\prime \prime} \quad 122^{\circ} 11^{\prime} 09^{\prime \prime} \quad 9-25-2001 \quad 1.8$

$48^{\circ} 04^{\prime} 33^{\prime \prime} \quad 122^{\circ} 11^{\prime} 21^{\prime \prime} \quad 9-24-2001 \quad .59$

$48^{\circ} 03^{\prime} 26^{\prime \prime} \quad 122^{\circ} 11^{\prime} 51^{\prime \prime} \quad 9-26-2001$

$48^{\circ} 05^{\prime} 07^{\prime \prime} \quad 122^{\circ} 14^{\prime} 54^{\prime \prime} \quad 9-25-2001$

$48^{\circ} 04^{\prime} 59^{\prime \prime} \quad 122^{\circ} 15^{\prime} 02^{\prime \prime} \quad 9-25-2001 \quad 0$

9-07-1977 $\quad 0.7$ 
Table 2. Low-flow measurement data for 410 selected sites in Washington, water years 1977 and 2001--Continued

\begin{tabular}{|c|c|c|c|c|c|c|c|}
\hline \multirow{2}{*}{$\begin{array}{l}\text { Gaging- } \\
\text { station } \\
\text { number }\end{array}$} & \multirow[b]{2}{*}{ Gaging-station name } & \multirow[b]{2}{*}{ Latitude } & \multirow[b]{2}{*}{ Longitude } & \multicolumn{2}{|c|}{ Water year 2001} & \multicolumn{2}{|c|}{ Water year 1977} \\
\hline & & & & Date & $\begin{array}{c}\text { Discharge, } \\
\text { in } \mathrm{ft}^{3} / \mathrm{s}\end{array}$ & Date & $\begin{array}{c}\text { Discharge, } \\
\text { in } \mathrm{ft}^{3} / \mathrm{s} \\
\end{array}$ \\
\hline \multicolumn{8}{|c|}{ Between Snohomish and Stillaguamish River Basins--Continued } \\
\hline 12157210 & Mission Creek Tributary \#2 near Tulalip,WA & $48^{\circ} 04^{\prime} 44^{\prime \prime}$ & $122^{\circ} 14^{\prime} 40^{\prime \prime}$ & $9-25-2001$ & 0.33 & 9-07-1977 & 0.3 \\
\hline 12158001 & Lake Shoecraft Outlet near Tulalip, WA & $48^{\circ} 07^{\prime} 23^{\prime \prime}$ & $122^{\circ} 18^{\prime} 28^{\prime \prime}$ & $9-25-2001$ & .10 & & \\
\hline 12158025 & East Branch Creek above Mary Shelton Lake near Tulalip, WA & $48^{\circ} 06^{\prime} 46^{\prime \prime}$ & $122^{\circ} 15^{\prime} 49^{\prime \prime}$ & $9-25-2001$ & 0 & 9-06-1977 & 0 \\
\hline \multicolumn{8}{|c|}{ Stillaguamish River Basin } \\
\hline --- & Lake Cavanaugh outlet near McMurray, WA & $48^{\circ} 18^{\prime} 40^{\prime \prime}$ & $121^{\circ} 58^{\prime} 34^{\prime \prime}$ & 9-20-2001 & 0 & & \\
\hline 12162500 & South Fork Stillaguamish River above Jim Creek near Arlington, WA & $48^{\circ} 10^{\prime} 44^{\prime \prime}$ & $122^{\circ} 05^{\prime} 07^{\prime \prime}$ & $9-20-2001$ & 205 & & \\
\hline 12164000 & Jim Creek near Arlington, WA & $48^{\circ} 11^{\prime} 00^{\prime \prime}$ & $122^{\circ} 04^{\prime} 40^{\prime \prime}$ & $9-19-2001$ & 18.1 & & \\
\hline 12165000 & Squire Creek near Darrington, WA & $48^{\circ} 16^{\prime} 14^{\prime \prime}$ & $121^{\circ} 40^{\prime} 16^{\prime \prime}$ & $9-17-2001$ & 56.4 & & \\
\hline 12166500 & Deer Creek at Oso, WA & $48^{\circ} 16^{\prime} 11^{\prime \prime}$ & $121^{\circ} 55^{\prime} 58^{\prime \prime}$ & $9-20-2001$ & 48.9 & & \\
\hline 12168500 & Pilchuck Creek near Bryant, WA & $48^{\circ} 15^{\prime} 57^{\prime \prime}$ & $122^{\circ} 09^{\prime} 50^{\prime \prime}$ & $9-20-2001$ & 28.6 & & \\
\hline \multicolumn{8}{|c|}{ Skagit River Basin } \\
\hline 12177500 & Stetattle Creek near Newhalem, WA & $48^{\circ} 43^{\prime} 03^{\prime \prime}$ & $121^{\circ} 09^{\prime} 01^{\prime \prime}$ & $9-17-2001$ & 56.4 & & \\
\hline 12180000 & Bacon Creek near Marblemount, WA & $48^{\circ} 35^{\prime} 20^{\prime \prime}$ & $121^{\circ} 23^{\prime} 45^{\prime \prime}$ & $9-17-2001$ & 133 & & \\
\hline 12182500 & Cascade River at Marblemount, WA & $48^{\circ} 31^{\prime} 37^{\prime \prime}$ & $121^{\circ} 24^{\prime} 47^{\prime \prime}$ & $9-18-2001$ & 374 & & \\
\hline 12184500 & Illabot Creek near Rockport, WA & $48^{\circ} 28^{\prime} 54^{\prime \prime}$ & $121^{\circ} 30^{\prime} 02^{\prime \prime}$ & $9-18-2001$ & 83.4 & & \\
\hline 12190000 & Jackman Creek near Concrete, WA & $48^{\circ} 31^{\prime} 26^{\prime \prime}$ & $121^{\circ} 42^{\prime} 46^{\prime \prime}$ & $9-18-2001$ & 19.8 & & \\
\hline 12190718 & Park Creek at Upper Bridge near Concrete, WA & $48^{\circ} 44^{\prime} 32^{\prime \prime}$ & $121^{\circ} 41^{\prime} 30^{\prime \prime}$ & $9-18-2001$ & 88.4 & & \\
\hline 12194500 & Finney Creek near Concrete, WA & $48^{\circ} 31^{\prime} 18^{\prime \prime}$ & $121^{\circ} 50^{\prime} 23^{\prime \prime}$ & $9-18-2001$ & 24.0 & & \\
\hline 12195000 & Grandy Creek near Concrete, WA & $48^{\circ} 31^{\prime} 59^{\prime \prime}$ & $121^{\circ} 53^{\prime} 03^{\prime \prime}$ & $9-18-2001$ & 9.1 & & \\
\hline 12196000 & Alder Creek near Hamilton, WA & $48^{\circ} 31^{\prime} 26^{\prime \prime}$ & $121^{\circ} 57^{\prime} 00^{\prime \prime}$ & $9-18-2001$ & 7.5 & & \\
\hline 12196500 & Day Creek near Lyman, WA & $48^{\circ} 30^{\prime} 07^{\prime \prime}$ & $122^{\circ} 02^{\prime} 48^{\prime \prime}$ & $9-19-2001$ & 21.2 & & \\
\hline 12197040 & Tank Creek near Lyman, WA & $48^{\circ} 31^{\prime} 35^{\prime \prime}$ & $122^{\circ} 06^{\prime} 30^{\prime \prime}$ & $9-19-2001$ & 0 & & \\
\hline 12197110 & Minkler Creek near Lyman, WA & $48^{\circ} 31^{\prime} 23^{\prime \prime}$ & $122^{\circ} 05^{\prime} 54^{\prime \prime}$ & $9-19-2001$ & 0 & & \\
\hline 12197680 & Black Creek near Minkler, WA & $48^{\circ} 32^{\prime} 00^{\prime \prime}$ & $122^{\circ} 07^{\prime} 13^{\prime \prime}$ & $9-19-2001$ & 0 & & \\
\hline 12197700 & Wiseman Creek near Lyman, WA & $48^{\circ} 31^{\prime} 48^{\prime \prime}$ & $122^{\circ} 08^{\prime} 12^{\prime \prime}$ & $9-19-2001$ & 1.7 & & \\
\hline 12200000 & East Fork Nookachamps Creek near Clear Lake, WA & $48^{\circ} 25^{\prime} 27^{\prime \prime}$ & $122^{\circ} 12^{\prime} 34^{\prime \prime}$ & $9-19-2001$ & 3.8 & & \\
\hline \multicolumn{8}{|c|}{ Between Skagit and Nooksack River Basins } \\
\hline 12201000 & Friday Creek near Burlington, WA & $48^{\circ} 34^{\prime} 26^{\prime \prime}$ & $122^{\circ} 20^{\prime} 19^{\prime \prime}$ & $9-19-2001$ & 13.0 & & \\
\hline \multicolumn{8}{|c|}{ Pend Oreille River Basin } \\
\hline --- & Le Clerc Creek near Metaline Falls, WA & $48^{\circ} 21^{\prime} 18^{\prime \prime}$ & $117^{\circ} 16^{\prime} 55^{\prime \prime}$ & $10-04-2001$ & 23.0 & & \\
\hline--- & Cee Cee Ah Creek near Metaline Falls, WA & $48^{\circ} 23^{\prime} 07^{\prime \prime}$ & $117^{\circ} 16^{\prime} 17^{\prime \prime}$ & $10-04-2001$ & 2.2 & & \\
\hline 12395900 & Davis Creek near Dalkena, WA & $48^{\circ} 13^{\prime} 50^{\prime \prime}$ & $117^{\circ} 17^{\prime} 17^{\prime \prime}$ & $10-04-2001$ & 3.5 & $8-31-1977$ & 2.4 \\
\hline 12396100 & Winchester Creek near Cusick, WA & $48^{\circ} 16^{\prime} 51^{\prime \prime}$ & $117^{\circ} 21^{\prime} 47^{\prime \prime}$ & $10-04-2001$ & 1.8 & $8-30-1977$ & 1.1 \\
\hline 12396450 & Little Muddy Creek at Ione, WA & $48^{\circ} 43^{\prime} 57^{\prime \prime}$ & $117^{\circ} 25^{\prime} 39^{\prime \prime}$ & $10-04-2001$ & .63 & & \\
\hline \multicolumn{8}{|c|}{ Between Pend Oreille and Kettle River Basins } \\
\hline--- & Little Sheep Creek at Velvet, WA & $48^{\circ} 59^{\prime} 09^{\prime \prime}$ & $117^{\circ} 49^{\prime} 38^{\prime \prime}$ & $10-04-2001$ & 1.5 & & \\
\hline 12399600 & Deep Creek near Northport, WA & $48^{\circ} 55^{\prime} 46^{\prime \prime}$ & $117^{\circ} 45^{\prime} 02^{\prime \prime}$ & $10-04-2001$ & 8.5 & 8-29-1977 & 10.3 \\
\hline 12400500 & Sheep Creek near Northport, WA & $48^{\circ} 56^{\prime} 26^{\prime \prime}$ & $117^{\circ} 46^{\prime} 54^{\prime \prime}$ & $10-04-2001$ & 21.0 & & \\
\hline \multicolumn{8}{|c|}{ Kettle River Basin } \\
\hline --- & Boulder Creek near Orient, WA & $48^{\circ} 50^{\prime} 09^{\prime \prime}$ & $118^{\circ} 11^{\prime} 07^{\prime \prime}$ & $10-05-2001$ & 4.0 & 8-23-1977 & 3.6 \\
\hline--- & Deadman Creek near Boyds, WA & $48^{\circ} 42^{\prime} 26^{\prime \prime}$ & $118^{\circ} 07^{\prime} 35^{\prime \prime}$ & $10-05-2001$ & 4.2 & & \\
\hline
\end{tabular}


Table 2. Low-flow measurement data for 410 selected sites in Washington, water years 1977 and 2001--Continued

\begin{tabular}{cl} 
Gaging- \\
station \\
number & \\
& \\
\hline-- & \multicolumn{1}{c}{ Gaging-station name } \\
--- & Mill Creek near Colville, WA \\
--- & Sottonwood Creek at mouth near Chewelah, WA \\
--- & Sheep Creek at Forest Center, WA \\
--- & Clugston Creek near Chewelah, WA \\
--- & Colville River at Chewelah, WA \\
--- & Sheep Creek near Valley, WA \\
--- & Colville River near Valley, WA \\
--- & Waitts Creek at mouth near Valley, WA \\
--- & Jump Off Joe Creek at mouth near Valley, WA \\
--- & Sherwood Creek at mouth near Chewelah, WA \\
--- & Bulldog Creek at mouth at Valley, WA \\
--- & Stensgar Creek at Addy, WA \\
--- & Addy Creek at Addy, WA \\
--- & Addy Creek at mouth at Addy, WA \\
--- & South Fork Chewelah Creek near Chewelah, WA \\
--- & Cottonwood Creek at mouth near Chewelah, WA \\
--- & North Fork Chewelah Creek near Chewelah, WA \\
--- & Huckleberry Creek at Wright Valley Road near Valley, WA \\
--- & Huckleberry Creek at mouth near Valley, WA \\
--- & Bulldog Creek near Valley, WA \\
--- & Little Pend Oreille River at mouth at Arden, WA \\
--- & East Branch South Fork Chewelah Creek near Chewelah, WA \\
--- & Thomason Creek at Chewelah, WA \\
--- & North Fork Chewelah Creek at Chewelah, WA \\
--- & Jump Off Joe Creek near Valley, WA \\
--- & Blue Creek near Bluecreek, WA \\
--- & Colville River at Arden, WA \\
--- & Colville River near Addy, WA \\
--- & Chewelah Creek at mouth at Chewelah, WA \\
--- & Stranger Creek at mouth near Addy, WA \\
--- & Little Pend Oreille River near Arden, WA \\
--- & Colville River near Colville, WA \\
--- & Paye Creek at Chewelah, WA \\
--- & Paye Creek at mouth at Chewelah, WA \\
--- & Deer Creek near Valley, WA \\
--- & Deer Creek at mouth near Valley, WA \\
--- & South Fork Chewelah Creek at Chewelah, WA \\
--- & Haller Creek near Chewelah, WA \\
--- & Thomason Creek near Chewelah, WA \\
&
\end{tabular}

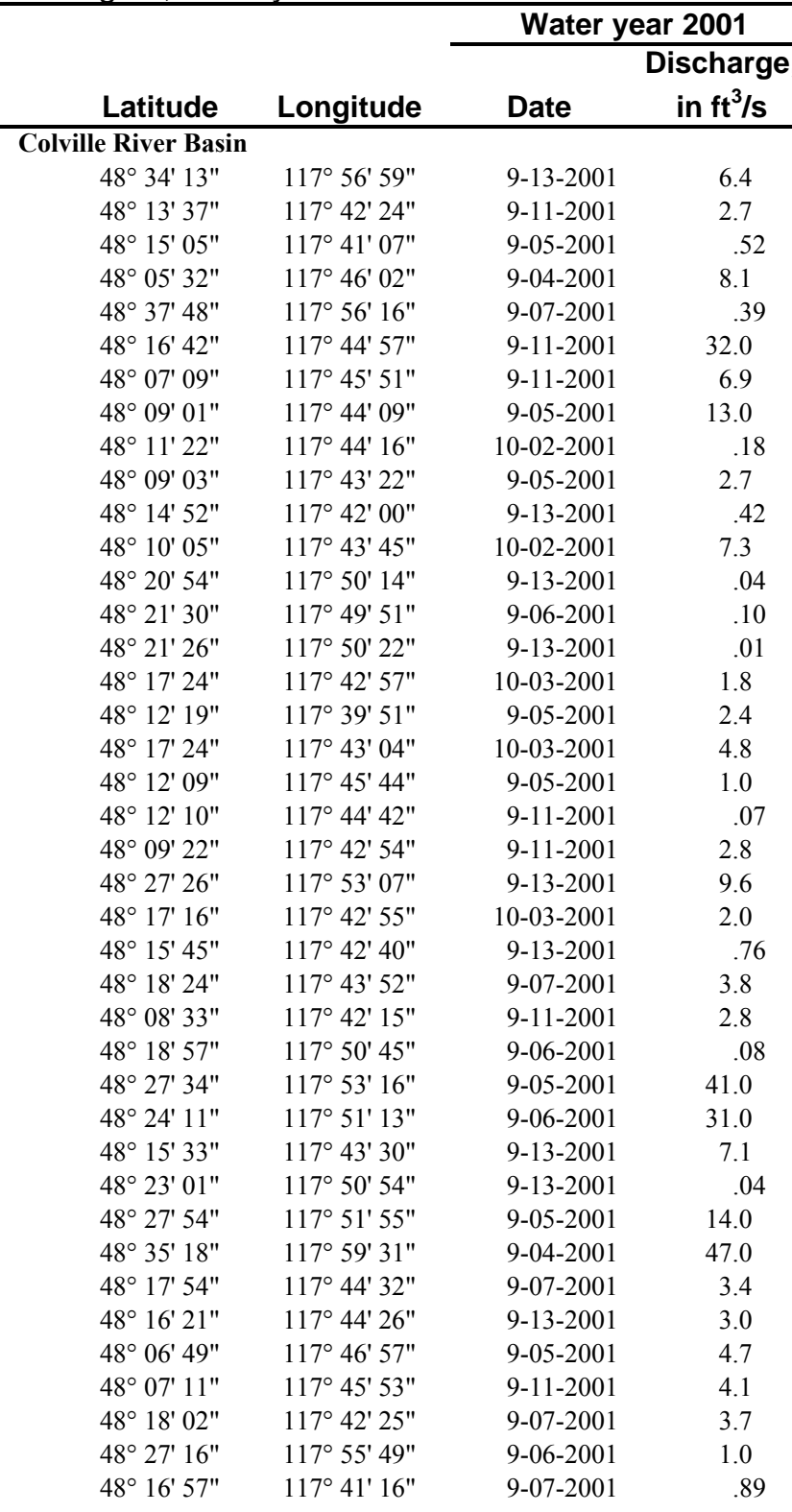


Table 2. Low-flow measurement data for 410 selected sites in Washington, water years 1977 and 2001--Continued

\begin{tabular}{|c|c|c|c|c|c|c|c|}
\hline \multirow{2}{*}{$\begin{array}{l}\text { Gaging- } \\
\text { station } \\
\text { number }\end{array}$} & \multirow[b]{2}{*}{ Gaging-station name } & \multirow[b]{2}{*}{ Latitude } & \multirow[b]{2}{*}{ Longitude } & \multicolumn{2}{|c|}{ Water year 2001} & \multicolumn{2}{|c|}{ Water year 1977} \\
\hline & & & & Date & $\begin{array}{c}\text { Discharge, } \\
\text { in } \mathrm{ft}^{3} / \mathrm{s}\end{array}$ & Date & $\begin{array}{c}\text { Discharge, } \\
\text { in } \mathrm{ft}^{3} / \mathrm{s}\end{array}$ \\
\hline \multicolumn{8}{|c|}{ Colville River Basin--Continued } \\
\hline--- & Stensgar Creek near Addy, WA & $48^{\circ} 20^{\prime} 23^{\prime \prime}$ & $117^{\circ} 52^{\prime} 18^{\prime \prime}$ & $9-06-2001$ & 0.33 & & \\
\hline --- & Haller Creek near Arden, WA & $48^{\circ} 28^{\prime} 15^{\prime \prime}$ & $117^{\circ} 53^{\prime} 39^{\prime \prime}$ & $9-13-2001$ & 0 & & \\
\hline --- & Mill Creek near Pinkney City, WA & $48^{\circ} 36^{\prime} 48^{\prime \prime}$ & $117^{\circ} 53^{\prime} 57^{\prime \prime}$ & 9-04-2001 & 3.8 & & \\
\hline --- & Grouse Creek near Valley, WA & $48^{\circ} 08^{\prime} 32^{\prime \prime}$ & $117^{\circ} 38^{\prime} 43^{\prime \prime}$ & $10-02-2001$ & .64 & 8-31-1977 & 0.38 \\
\hline --- & Waitts Creek near Valley, WA & $48^{\circ} 11^{\prime} 03^{\prime \prime}$ & $117^{\circ} 45^{\prime} 28^{\prime \prime}$ & $10-02-2001$ & .11 & & \\
\hline --- & Gold Creek near Colville, WA & $48^{\circ} 34^{\prime} 19^{\prime \prime}$ & $117^{\circ} 57^{\prime} 57^{\prime \prime}$ & $9-07-2001$ & 0 & & \\
\hline --- & Blue Creek at mouth near Bluecreek, WA & $48^{\circ} 19^{\prime} 09^{\prime \prime}$ & $117^{\circ} 49^{\prime} 09^{\prime \prime}$ & $9-13-2001$ & .04 & & \\
\hline 12407500 & Sheep Creek at Springdale, WA & $48^{\circ} 03^{\prime} 27^{\prime \prime}$ & $117^{\circ} 45^{\prime} 07^{\prime \prime}$ & 9-04-2001 & 5.5 & & \\
\hline 12407520 & Deer Creek near Valley, WA & $48^{\circ} 07^{\prime} 05^{\prime \prime}$ & $117^{\circ} 47^{\prime} 55^{\prime \prime}$ & $10-03-2001$ & 5.4 & 8-31-1977 & 3.9 \\
\hline 12407560 & Huckleberry Creek near Valley, WA & $48^{\circ} 12^{\prime} 28^{\prime \prime}$ & $117^{\circ} 45^{\prime} 52^{\prime \prime}$ & $10-02-2001$ & .22 & 8-31-1977 & .46 \\
\hline 12407580 & Cottonwood Creek near Chewelah, WA & $48^{\circ} 13^{\prime} 22^{\prime \prime}$ & $117^{\circ} 42^{\prime} 16^{\prime \prime}$ & $10-03-2001$ & 2.9 & & \\
\hline 12407700 & Chewelah Creek at Chewelah, WA & $48^{\circ} 17^{\prime} 00^{\prime \prime}$ & $117^{\circ} 42^{\prime} 53^{\prime \prime}$ & $10-03-2001$ & 9.6 & 8-30-1977 & 5.6 \\
\hline 12408000 & Colville River at Blue Creek, WA & $48^{\circ} 19^{\prime} 11^{\prime \prime}$ & $117^{\circ} 49^{\prime} 09^{\prime \prime}$ & $9-05-2001$ & 31.0 & 8-30-1977 & 27.2 \\
\hline 12408120 & Stranger Creek near Addy, WA & $48^{\circ} 22^{\prime} 19^{\prime \prime}$ & $117^{\circ} 51^{\prime} 33^{\prime \prime}$ & $10-03-2001$ & .75 & 8-30-1977 & 60 \\
\hline 12408300 & Little Pend Oreille River near Colville, WA & $48^{\circ} 27^{\prime} 57^{\prime \prime}$ & $117^{\circ} 44^{\prime} 57^{\prime \prime}$ & $10-03-2001$ & 12.0 & & \\
\hline 12408420 & Haller Creek near Arden, WA & $48^{\circ} 28^{\prime} 01^{\prime \prime}$ & $117^{\circ} 54^{\prime} 28^{\prime \prime}$ & $10-03-2001$ & .63 & $8-30-1977$ & .41 \\
\hline 12408500 & Mill Creek near Colville, WA & $48^{\circ} 34^{\prime} 43^{\prime \prime}$ & $117^{\circ} 52^{\prime} 00^{\prime \prime}$ & 9-04-2001 & 4.7 & 8-30-1977 & 5.2 \\
\hline \multicolumn{8}{|c|}{ Between Colville and Spokane River Basins } \\
\hline--- & Nine Mile Creek near Fruitland, WA & $48^{\circ} 02^{\prime} 49^{\prime \prime}$ & $118^{\circ} 26^{\prime} 09^{\prime \prime}$ & $10-16-2001$ & 2.6 & & \\
\hline--- & Wilmont Creek near Fruitland, WA & $48^{\circ} 04^{\prime} 33^{\prime \prime}$ & $118^{\circ} 19^{\prime} 32^{\prime \prime}$ & $10-16-2001$ & .02 & & \\
\hline--- & Barnaby Creek near Rice, WA & $48^{\circ} 26^{\prime} 03^{\prime \prime}$ & $118^{\circ} 13^{\prime} 35^{\prime \prime}$ & $10-05-2001$ & 2.8 & $8-22-1977$ & .75 \\
\hline--- & Stranger Creek near Inchelium, WA & $48^{\circ} 15^{\prime} 51^{\prime \prime}$ & $118^{\circ} 17^{\prime} 02^{\prime \prime}$ & $10-05-2001$ & .85 & & \\
\hline 12409500 & Hall Creek at Inchelium, WA & $48^{\circ} 18^{\prime} 40^{\prime \prime}$ & $118^{\circ} 12^{\prime} 42^{\prime \prime}$ & $10-04-2001$ & 6.2 & & \\
\hline \multicolumn{8}{|c|}{ Spokane River Basin } \\
\hline--- & Little Chamokane Creek near Long Lake, WA & $47^{\circ} 51^{\prime} 12^{\prime \prime}$ & $117^{\circ} 52^{\prime} 47^{\prime \prime}$ & $10-10-2001$ & .50 & & \\
\hline--- & Hangman Creek at Tekoa, WA & $47^{\circ} 13^{\prime} 34^{\prime \prime}$ & $117^{\circ} 04^{\prime} 49^{\prime \prime}$ & $10-11-2001$ & 60 & $8-26-1977$ & .25 \\
\hline--- & Dry Creek near Milan, WA & $47^{\circ} 59^{\prime} 10^{\prime \prime}$ & $117^{\circ} 17^{\prime} 42^{\prime \prime}$ & $10-05-2001$ & 2.6 & $8-29-1977$ & 1.6 \\
\hline--- & West Branch Little Spokane River near Chattaroy, WA & $47^{\circ} 59^{\prime} 49^{\prime \prime}$ & $117^{\circ} 20^{\prime} 58^{\prime \prime}$ & $10-05-2001$ & 4.2 & 9-06-1977 & .47 \\
\hline--- & Little Hangman Creek at Tekoa, WA & $47^{\circ} 13^{\prime} 34^{\prime \prime}$ & $117^{\circ} 04^{\prime} 21^{\prime \prime}$ & $10-11-2001$ & .43 & $8-26-1977$ & .13 \\
\hline 12425500 & Deep Creek near Spokane, WA & $47^{\circ} 40^{\prime} 56^{\prime \prime}$ & $117^{\circ} 40^{\prime} 37^{\prime \prime}$ & $10-10-2001$ & 1.6 & 9-01-1977 & 41 \\
\hline 12427000 & Little Spokane River at Elk, WA & $48^{\circ} 01^{\prime} 19^{\prime \prime}$ & $117^{\circ} 16^{\prime} 22^{\prime \prime}$ & $10-05-2001$ & 34.0 & 8-16-1977 & 27.3 \\
\hline 12429600 & Deer Creek near Chattaroy, WA & $47^{\circ} 53^{\prime} 27^{\prime \prime}$ & $117^{\circ} 20^{\prime} 09^{\prime \prime}$ & $10-05-2001$ & .17 & 8-29-1977 & 0 \\
\hline 12430100 & Dragoon Creek at mouth near Chattaroy, WA & $47^{\circ} 52^{\prime} 33^{\prime \prime}$ & $117^{\circ} 22^{\prime} 11^{\prime \prime}$ & $10-05-2001$ & 17.0 & 8-31-1977 & 14.1 \\
\hline 12430400 & Deadman Creek below Highway 195 near Mead, WA & $47^{\circ} 46^{\prime} 52^{\prime \prime}$ & $117^{\circ} 21^{\prime} 52^{\prime \prime}$ & $10-05-2001$ & 3.2 & & \\
\hline 12430500 & Deep Creek at Colbert, WA & $47^{\circ} 49^{\prime} 16^{\prime \prime}$ & $117^{\circ} 20^{\prime} 47^{\prime \prime}$ & $10-05-2001$ & 0 & 8-29-1977 & 0 \\
\hline \multicolumn{8}{|c|}{ Between Spokane and Sanpoil River Basins } \\
\hline--- & Hawk Creek near Lincoln, WA & $47^{\circ} 48^{\prime} 54^{\prime \prime}$ & $118^{\circ} 18^{\prime} 59^{\prime \prime}$ & $10-10-2001$ & 7.9 & & \\
\hline \multicolumn{8}{|c|}{ Sanpoil River Basin } \\
\hline 12434110 & West Fork Sanpoil River near Republic, WA & $48^{\circ} 27^{\prime} 32^{\prime \prime}$ & $118^{\circ} 45^{\prime} 01^{\prime \prime}$ & $10-01-2001$ & 5.1 & & \\
\hline 12434500 & Sanpoil River near Keller, WA & $48^{\circ} 06^{\prime} 25^{\prime \prime}$ & $118^{\circ} 41^{\prime} 55^{\prime \prime}$ & $10-01-2001$ & 16.0 & 8-26-1977 & 9.7 \\
\hline
\end{tabular}


Table 2. Low-flow measurement data for 410 selected sites in Washington, water years 1977 and 2001--Continued

\begin{tabular}{|c|c|}
\hline $\begin{array}{c}\text { Gaging- } \\
\text { station } \\
\text { number }\end{array}$ & Gaging-station name \\
\hline 12437505 & Nespelem River below millpond at Nespelem, WA \\
\hline--- & Bonaparte Creek at Tonasket, WA \\
\hline --- & Loup Loup Creek at Malott, WA \\
\hline --- & Omak Creek near Omak, WA \\
\hline--- & Aeneas Creek near Tonasket, WA \\
\hline--- & Antoine Creek near Ellisford, WA \\
\hline--- & Palmer Creek near Nighthawk, WA \\
\hline--- & Johnson Creek near Riverside, WA \\
\hline 12442000 & Toats Coulee Creek near Loomis, WA \\
\hline 12442300 & Sinlahekin Creek above Chopaka Creek near Loomis, WA \\
\hline
\end{tabular}$$
-
$$$$
\text { --- }
$$$$
-
$$$$
-
$$$$
\text { --- }
$$$$
---
$$$$
---
$$$$
\text { --- }
$$$$
\text { --- }
$$$$
---
$$$$
\text { --- }
$$

Methow River below Beaver Creek near Twisp, WA

--- $\quad$ Robinson Creek near Mazama, WA

--- $\quad$ Twisp River below Poorman Creek near Twisp, WA

--- $\quad$ Methow River above Early Winters Creek near Mazama, WA

12447350 Methow River above Robinson Creek near Mazama, WA

12447384 Goat Creek near Mazama, WA

12447385 Methow River at Weeman Bridge near Mazama, WA

12447386 Methow River above Wolf Creek near Winthrop, WA

12447394 Lake Creek near Winthrop, WA

12447450 Chewuch River at Eightmile Ranch near Winthrop, WA

12447500 Chewuch River below Boulder Creek near Winthrop, WA

12448850 Twisp River above Buttermilk Creek near Twisp, WA
Water year 2001

Discharge,

Between Sanpoil and Okanogan River Basins

$48^{\circ} 09^{\prime} 53^{\prime \prime} \quad 118^{\circ} 58^{\prime} 49^{\prime \prime}$

Date

$119^{\circ} 26^{\prime} 33^{\prime \prime}$

$48^{\circ} 42^{\prime} 04^{\prime \prime}$

$48^{\circ} 16^{\prime} 57^{\prime \prime} \quad 119^{\circ} 42^{\prime} 31^{\prime \prime}$

$48^{\circ} 21^{\prime} 58^{\prime \prime} \quad 119^{\circ} 26^{\prime} 50^{\prime \prime}$

$48^{\circ} 39^{\prime} 33^{\prime \prime} \quad 119^{\circ} 28^{\prime} 45^{\prime \prime}$

$48^{\circ} 45^{\prime} 33^{\prime \prime} \quad 119^{\circ} 24^{\prime} 33^{\prime \prime}$

$48^{\circ} 55^{\prime} 24^{\prime \prime} \quad 119^{\circ} 39^{\prime} 21^{\prime \prime}$

$48^{\circ} 29^{\prime} 32^{\prime \prime} \quad 119^{\circ} 32^{\prime} 09^{\prime \prime}$

$48^{\circ} 50^{\prime} 00^{\prime \prime} \quad 119^{\circ} 41^{\prime} 36^{\prime \prime}$

$48^{\circ} 51^{\prime} 05^{\prime \prime} \quad 119^{\circ} 38^{\prime} 57^{\prime \prime}$

Methow River Basin

$48^{\circ} 30^{\prime} 19^{\prime \prime}$

$48^{\circ} 05^{\prime} 40^{\prime \prime}$

$48^{\circ} 11^{\prime} 17^{\prime \prime}$

$48^{\circ} 13^{\prime} 43^{\prime \prime}$

$48^{\circ} 34^{\prime} 42^{\prime \prime}$

$48^{\circ} 36^{\prime} 14^{\prime \prime}$

$48^{\circ} 25^{\prime} 06^{\prime \prime}$

$48^{\circ} 22^{\prime} 46^{\prime \prime}$

$48^{\circ} 23^{\prime} 44^{\prime \prime}$

$48^{\circ} 27^{\prime} 00^{\prime \prime}$

$48^{\circ} 20^{\prime} 43^{\prime \prime}$

$48^{\circ} 22^{\prime} 13^{\prime \prime}$

$48^{\circ} 22^{\prime} 20^{\prime \prime}$

$48^{\circ} 21^{\prime} 50^{\prime \prime}$

$48^{\circ} 19^{\prime} 21^{\prime \prime}$

$48^{\circ} 39^{\prime} 41^{\prime \prime}$

$48^{\circ} 22^{\prime} 06^{\prime \prime}$

$48^{\circ} 36^{\prime} 03^{\prime \prime}$

$48^{\circ} 39^{\prime} 32^{\prime \prime}$

$48^{\circ} 34^{\prime} 52^{\prime \prime}$

$48^{\circ} 32^{\prime} 39^{\prime \prime}$

$48^{\circ} 29^{\prime} 27^{\prime \prime}$

$48^{\circ} 45^{\prime} 23^{\prime \prime}$

$48^{\circ} 36^{\prime} 07^{\prime \prime}$

$48^{\circ} 34^{\prime} 35^{\prime \prime}$

$120^{\circ} 16^{\prime} 43^{\prime \prime}$

$120^{\circ} 01^{\prime} 11^{\prime \prime}$

$120^{\circ} 05^{\prime} 46^{\prime \prime}$

$120^{\circ} 06^{\prime} 52^{\prime \prime}$

$120^{\circ} 09^{\prime} 53^{\prime \prime}$

$120^{\circ} 09^{\prime} 51^{\prime \prime}$

$120^{\circ} 08^{\prime} 34^{\prime \prime}$

$120^{\circ} 14^{\prime} 40^{\prime \prime}$

$120^{\circ} 08^{\prime} 21^{\prime \prime}$

$120^{\circ} 09^{\prime} 48^{\prime \prime}$

$120^{\circ} 05^{\prime} 49^{\prime \prime}$

$120^{\circ} 11^{\prime} 19^{\prime \prime}$

$120^{\circ} 12^{\prime} 05^{\prime \prime}$

$120^{\circ} 20^{\prime} 11^{\prime \prime}$

$120^{\circ} 03^{\prime} 50^{\prime \prime}$

$120^{\circ} 32^{\prime} 26^{\prime \prime}$

$120^{\circ} 10^{\prime} 37^{\prime \prime}$

$120^{\circ} 26^{\prime} 23^{\prime \prime}$

$120^{\circ} 32^{\prime} 31^{\prime \prime}$

$120^{\circ} 22^{\prime} 46^{\prime \prime}$

$120^{\circ} 19^{\prime} 26^{\prime \prime}$

$120^{\circ} 13^{\prime} 56^{\prime \prime}$

$120^{\circ} 08^{\prime} 08^{\prime \prime}$

$120^{\circ} 09^{\prime} 49^{\prime \prime}$

$120^{\circ} 10^{\prime} 34^{\prime \prime}$

$120^{\circ} 24^{\prime} 18^{\prime \prime}$
10-11-2001

10-02-2001

10-03-2001

10-03-2001

$10-10-2001$

$10-02-2001$

10-02-2001

10-03-2001

10-02-2001

10-02-2001

9-12-2001

9-12-2001

9-21-2001

9-20-2001

9-19-2001

9-19-2001

9-12-2001

9-11-2001

9-12-2001

9-12-2001

9-12-2001

9-11-2001

9-11-2001

9-11-2001

9-12-2001

9-13-2001

9-11-2001

9-13-2001

9-18-2001

9-18-2001

9-12-2001

9-19-2001

9-17-2001

9-19-2001

9-13-2001

9-20-2001 in $\mathrm{ft}^{3} / \mathrm{s}$

Water year 1977

Discharge

in $\mathrm{ft}^{3} / \mathrm{s}$

$\begin{array}{lll}7.6 & 8-26-1977\end{array}$

.36

2.6
2.3
.73

.73

.28

3.7

8.2
7.5

8-24-1977

8-24-1977

112

231
4.1

2

2.2
3.4

12.0

107

151
140

188

24.0

25.0

25.0

218

6.0
28.0
3.5

3.5

3.5
13.0
.44

29.0

103

6.1
35.0

35.0

45.0 
Table 2. Low-flow measurement data for 410 selected sites in Washington, water years 1977 and 2001--Continued

\begin{tabular}{|c|c|}
\hline $\begin{array}{c}\text { Gaging- } \\
\text { station } \\
\text { number }\end{array}$ & Gaging-station name \\
\hline--- & Mitchell Creek at mouth near Manson, WA \\
\hline--- & Safety Harbor Creek near Lucerne, WA \\
\hline--- & Prince Creek near Lucerne, WA \\
\hline--- & Fish Creek near Lucerne, WA \\
\hline--- & North 25 Mile Creek near Manson, WA \\
\hline--- & Railroad Creek at Lucerne, WA \\
\hline--- & Poison Creek near Manson, WA \\
\hline--- & First Creek near Manson, WA \\
\hline 12451610 & Falls Creek near Manson, WA \\
\hline 12451620 & Grade Creek near Manson, WA \\
\hline 12451660 & Mitchell Creek near Manson, WA \\
\hline 12452880 & Tillicum Creek near Ardenvoir, WA \\
\hline 12452890 & Mad River at Ardenvoir, WA \\
\hline--- & Alder Creek near Plain, WA \\
\hline--- & Doctor Creek near Leavenworth, WA \\
\hline --- & Hansel Creek near Peshastin, WA \\
\hline --- & Jack Creek near Leavenworth, WA \\
\hline--- & Deep Creek near Plain, WA \\
\hline --- & Beaver Creek at Plain, WA \\
\hline--- & Icicle Creek above Black Pine Creek near Leavenworth, WA \\
\hline--- & Black Pine Creek near Leavenworth, WA \\
\hline --- & Goose Creek near Plain, WA \\
\hline--- & Trout Creek near Leavenworth, WA \\
\hline--- & Negro Creek near Peshastin, WA \\
\hline --- & Big Meadow Creek near Plain, WA \\
\hline --- & Ida Creek near Leavenworth, WA \\
\hline --- & Johnny Creek near Leavenworth, WA \\
\hline --- & Bridge Creek near Leavenworth, WA \\
\hline--- & Chumstick Creek near Leavenworth, WA \\
\hline--- & Peshastin Creek near Peshastin, WA \\
\hline--- & Derby Canyon near Peshastin, WA \\
\hline--- & Scotty Creek near Blewett, WA \\
\hline--- & Shaser Creek near Blewett, WA \\
\hline--- & Ingalls Creek near Leavenworth, WA \\
\hline--- & Lake Creek near Telma, WA \\
\hline--- & White River near Telma, WA \\
\hline--- & Panther Creek near Telma, WA \\
\hline--- & Napeequa River near Telma, WA \\
\hline
\end{tabular}

\begin{tabular}{|c|c|c|c|c|c|}
\hline \multirow[b]{2}{*}{ Latitude } & \multirow[b]{2}{*}{ Longitude } & \multicolumn{2}{|c|}{ Water year 2001} & \multicolumn{2}{|c|}{ Water year 1977} \\
\hline & & Date & $\begin{array}{c}\text { Discharge, } \\
\text { in } \mathrm{ft}^{3} / \mathrm{s}\end{array}$ & Date & $\begin{array}{c}\text { Discharge, } \\
\text { in } \mathrm{ft}^{3} / \mathrm{s} \\
\end{array}$ \\
\hline \multicolumn{6}{|l|}{ Chelan River Basin } \\
\hline $47^{\circ} 58^{\prime} 10^{\prime \prime}$ & $120^{\circ} 11^{\prime} 32^{\prime \prime}$ & $10-22-2001$ & 0.76 & & \\
\hline $48^{\circ} 02^{\prime} 46^{\prime \prime}$ & $120^{\circ} 22^{\prime} 34^{\prime \prime}$ & $10-22-2001$ & 3.0 & & \\
\hline $48^{\circ} 08^{\prime} 49^{\prime \prime}$ & $120^{\circ} 29^{\prime} 50^{\prime \prime}$ & $10-22-2001$ & 9.9 & & \\
\hline $48^{\circ} 14^{\prime} 10^{\prime \prime}$ & $120^{\circ} 36^{\prime} 58^{\prime \prime}$ & $10-22-2001$ & 6.2 & & \\
\hline $47^{\circ} 59^{\prime} 32^{\prime \prime}$ & $120^{\circ} 15^{\prime} 44^{\prime \prime}$ & $10-29-2001$ & 4.9 & & \\
\hline $48^{\circ} 12^{\prime} 03^{\prime \prime}$ & $120^{\circ} 35^{\prime} 40^{\prime \prime}$ & $10-22-2001$ & 54.0 & & \\
\hline $48^{\circ} 02^{\prime} 05^{\prime \prime}$ & $120^{\circ} 13^{\prime} 10^{\prime \prime}$ & $10-11-2001$ & .38 & & \\
\hline $47^{\circ} 52^{\prime} 25^{\prime \prime}$ & $120^{\circ} 12^{\prime} 02^{\prime \prime}$ & $10-29-2001$ & 3.0 & & \\
\hline $48^{\circ} 05^{\prime} 07^{\prime \prime}$ & $120^{\circ} 19^{\prime} 28^{\prime \prime}$ & $10-11-2001$ & 1.1 & $8-24-1977$ & 0.8 \\
\hline $48^{\circ} 03^{\prime} 35^{\prime \prime}$ & $120^{\circ} 15^{\prime} 30^{\prime \prime}$ & $10-11-2001$ & 1.0 & $8-24-1977$ & .51 \\
\hline $47^{\circ} 59^{\prime} 44^{\prime \prime}$ & $120^{\circ} 09^{\prime} 38^{\prime \prime}$ & $10-11-2001$ & 0 & & \\
\hline \multicolumn{6}{|l|}{ Entiat River Basin } \\
\hline $47^{\circ} 43^{\prime} 24^{\prime \prime}$ & $120^{\circ} 26^{\prime} 24^{\prime \prime}$ & $9-19-2001$ & .50 & & \\
\hline $47^{\circ} 44^{\prime} 12^{\prime \prime}$ & $120^{\circ} 22^{\prime} 07^{\prime \prime}$ & $10-11-2001$ & 15.0 & & \\
\hline \multicolumn{6}{|c|}{ Wenatchee River Basin } \\
\hline $47^{\circ} 50^{\prime} 54^{\prime \prime}$ & $120^{\circ} 39^{\prime} 35^{\prime \prime}$ & $10-09-2001$ & .70 & $9-20-1977$ & 1.2 \\
\hline $47^{\circ} 36^{\prime} 25^{\prime \prime}$ & $120^{\circ} 51^{\prime} 58^{\prime \prime}$ & $10-05-2001$ & 0 & & \\
\hline $47^{\circ} 28^{\prime} 16^{\prime \prime}$ & $120^{\circ} 39^{\prime} 25^{\prime \prime}$ & $10-10-2001$ & .35 & & \\
\hline $47^{\circ} 36^{\prime} 30^{\prime \prime}$ & $120^{\circ} 54^{\prime} 30^{\prime \prime}$ & $10-05-2001$ & 7.6 & & \\
\hline $47^{\circ} 49^{\prime} 11^{\prime \prime}$ & $120^{\circ} 38^{\prime} 04^{\prime \prime}$ & $10-10-2001$ & .27 & $9-21-1977$ & .33 \\
\hline $47^{\circ} 45^{\prime} 52^{\prime \prime}$ & $120^{\circ} 39^{\prime} 22^{\prime \prime}$ & $10-10-2001$ & 1.6 & $9-21-1977$ & 1.4 \\
\hline $47^{\circ} 36^{\prime} 45^{\prime \prime}$ & $120^{\circ} 56^{\prime} 44^{\prime \prime}$ & $10-05-2001$ & 21.0 & & \\
\hline $47^{\circ} 36^{\prime} 41^{\prime \prime}$ & $120^{\circ} 56^{\prime} 43^{\prime \prime}$ & $10-05-2001$ & 0 & & \\
\hline $47^{\circ} 50^{\prime} 20^{\prime \prime}$ & $120^{\circ} 38^{\prime} 50^{\prime \prime}$ & $10-09-2001$ & .17 & & \\
\hline $47^{\circ} 36^{\prime} 22^{\prime \prime}$ & $120^{\circ} 53^{\prime} 37^{\prime \prime}$ & $10-05-2001$ & 2.7 & & \\
\hline $47^{\circ} 26^{\prime} 36^{\prime \prime}$ & $120^{\circ} 39^{\prime} 42^{\prime \prime}$ & $10-10-2001$ & 1.9 & $9-19-1977$ & 2.9 \\
\hline $47^{\circ} 52^{\prime} 03^{\prime \prime}$ & $120^{\circ} 41^{\prime} 48^{\prime \prime}$ & $10-04-2001$ & .74 & & \\
\hline $47^{\circ} 36^{\prime} 25^{\prime \prime}$ & $120^{\circ} 50^{\prime} 57^{\prime \prime}$ & $10-05-2001$ & .02 & & \\
\hline $47^{\circ} 35^{\prime} 53^{\prime \prime}$ & $120^{\circ} 49^{\prime} 05^{\prime \prime}$ & $10-05-2001$ & .43 & & \\
\hline $47^{\circ} 33^{\prime} 40^{\prime \prime}$ & $120^{\circ} 46^{\prime} 48^{\prime \prime}$ & $10-10-2001$ & .16 & & \\
\hline $47^{\circ} 36^{\prime} 17^{\prime \prime}$ & $120^{\circ} 38^{\prime} 54^{\prime \prime}$ & $10-09-2001$ & 1.3 & 9-20-1977 & 3.9 \\
\hline $47^{\circ} 33^{\prime} 08^{\prime \prime}$ & $120^{\circ} 36^{\prime} 11^{\prime \prime}$ & $10-10-2001$ & 16.0 & & \\
\hline $47^{\circ} 34^{\prime} 10^{\prime \prime}$ & $120^{\circ} 35^{\prime} 14^{\prime \prime}$ & $10-09-2001$ & 0 & 9-19-1977 & 4.1 \\
\hline $47^{\circ} 22^{\prime} 39^{\prime \prime}$ & $120^{\circ} 38^{\prime} 45^{\prime \prime}$ & $10-10-2001$ & .10 & 9-19-1977 & .24 \\
\hline $47^{\circ} 23^{\prime} 27^{\prime \prime}$ & $120^{\circ} 39^{\prime} 32^{\prime \prime}$ & $10-10-2001$ & .51 & 9-19-1977 & .71 \\
\hline $47^{\circ} 27^{\prime} 47^{\prime \prime}$ & $120^{\circ} 39^{\prime} 42^{\prime \prime}$ & $10-10-2001$ & 11.0 & 9-19-1977 & 23.8 \\
\hline $47^{\circ} 52^{\prime} 34^{\prime \prime}$ & $121^{\circ} 02^{\prime} 08^{\prime \prime}$ & $10-03-2001$ & 4.9 & & \\
\hline $47^{\circ} 57^{\prime} 49^{\prime \prime}$ & $120^{\circ} 56^{\prime} 39^{\prime \prime}$ & $10-01-2001$ & 77.0 & & \\
\hline $47^{\circ} 56^{\prime} 23^{\prime \prime}$ & $120^{\circ} 55^{\prime} 45^{\prime \prime}$ & $10-02-2001$ & 5.7 & & \\
\hline $47^{\circ} 55^{\prime} 16^{\prime \prime}$ & $120^{\circ} 53^{\prime} 42^{\prime \prime}$ & $10-02-2001$ & 41.0 & & \\
\hline
\end{tabular}


Table 2. Low-flow measurement data for 410 selected sites in Washington, water years 1977 and 2001--Continued

\begin{tabular}{cl}
$\begin{array}{c}\text { Gaging- } \\
\text { station } \\
\text { number }\end{array}$ & \multicolumn{1}{c}{ Gaging-station name } \\
\hline-- & Canyon Creek near Telma, WA \\
--- & Fish Creek near Telma, WA \\
--- & Chikamin Creek near Telma, WA \\
--- & Theseus Creek near Telma, WA \\
--- & Little Wenatchee River above Wenatchee Lake near Telma, WA \\
--- & Minnow Creek near Plain, WA \\
--- & Rock Creek near Telma, WA \\
--- & Cady Creek near Telma, WA \\
--- & Nason Creek near Coles Corner, WA \\
--- & Rainy Creek near Telma, WA \\
12456000 & Phelps Creek near Plain, WA \\
12456300 & Brush Creek near Telma, WA \\
12457500 & Chiwaukum Creek near Chiwaukum, WA \\
12457900 & Chatter Creek near Leavenworth, WA \\
12459400 & Tronsen Creek near Peshastin, WA \\
12461100 & East Branch Mission Creek near Cashmere, WA \\
12461400 & Mission Creek above Sand Creek near Cashmere, WA \\
12461500 & Sand Creek near Cashmere, WA \\
12462000 & Mission Creek at Cashmere, WA
\end{tabular}

Latitude Longitude

Water year 2001

Wenatchee River Basin--Continued

$47^{\circ} 54^{\prime} 26^{\prime \prime}$

$120^{\circ} 53^{\prime} 42^{\prime \prime}$

$47^{\circ} 54^{\prime} 30^{\prime \prime} \quad 121^{\circ} 05^{\circ} 03^{\prime \prime}$

$47^{\circ} 52^{\prime} 23^{\prime \prime} \quad 121^{\circ} 01^{\prime} 02^{\prime \prime}$

$47^{\circ} 50^{\prime} 01^{\prime \prime} \quad 120^{\circ} 50^{\prime} 15^{\prime \prime}$

$47^{\circ} 54^{\prime} 35^{\prime \prime} \quad 120^{\circ} 43^{\prime} 15^{\prime \prime}$

$47^{\circ} 58^{\prime} 12^{\prime \prime} \quad 120^{\circ} 47^{\prime} 22^{\prime \prime}$

$121^{\circ} 05^{\prime} 37^{\prime \prime}$

$47^{\circ} 47^{\prime} 25^{\prime \prime} \quad 120^{\circ} 42^{\prime} 59^{\prime \prime}$

$47^{\circ} 51^{\prime} 01^{\prime \prime} \quad 120^{\circ} 57^{\prime} 41^{\prime \prime}$

$48^{\circ} 04^{\prime} 23^{\prime \prime} \quad 120^{\circ} 51^{\prime} 02^{\prime \prime}$

$47^{\circ} 53^{\prime} 19^{\prime \prime} \quad 120^{\circ} 43^{\prime} 19^{\prime \prime}$

$47^{\circ} 40^{\prime} 49^{\prime \prime} \quad 120^{\circ} 43^{\prime} 54^{\prime \prime}$

$47^{\circ} 36^{\prime} 28^{\prime \prime} \quad 120^{\circ} 52^{\prime} 56^{\prime \prime}$

$47^{\circ} 20^{\prime} 23^{\prime \prime} \quad 120^{\circ} 34^{\prime} 03^{\prime \prime}$

$47^{\circ} 22^{\prime} 50^{\prime \prime} \quad 120^{\circ} 29^{\prime} 20^{\prime \prime}$

$47^{\circ} 25^{\prime} 47^{\prime \prime} \quad 120^{\circ} 30^{\prime} 32^{\prime \prime}$

$47^{\circ} 25^{\prime} 47^{\prime \prime} \quad 120^{\circ} 30^{\prime} 29^{\prime \prime}$

$47^{\circ} 30^{\prime} 59^{\prime \prime}$

$120^{\circ} 28^{\prime} 34^{\prime \prime}$

Between Wenatchee River and Crab Creek Basins

$47^{\circ} 25^{\prime} 11^{\prime \prime} \quad 119^{\circ} 55^{\prime} 00^{\prime \prime}$

$47^{\circ} 37^{\prime} 02^{\prime \prime} \quad 120^{\circ} 00^{\prime} 31^{\prime \prime}$

$47^{\circ} 35^{\prime} 01^{\prime \prime} \quad 120^{\circ} 01^{\prime} 23^{\prime \prime}$

12462800 Moses Creek at Douglas, WA

12463000 Douglas Creek near Alstown, WA

--- $\quad$ Goose Creek at Wilbur, WA

--- $\quad$ Corbett Draw near Almira, WA

12464800 Coal Creek at Mohler, WA

12465400 Wilson Creek below Corbett Draw near Almira, WA

--- Logy Creek near Toppenish, WA

--- Lateral 1 near Parker, WA

--- $\quad$ Yakima River at Donald, WA

--- Yakima River near Donald, WA

--- Yakima River near Toppenish, WA

--- $\quad$ North Fork Tieton River near White Pass, WA

--- Paris Creek near Salmon Ia Sac Guard Station, WA

--- $\quad$ Cle Elum River above Cle Elum Lake, WA

--- Yakima River below Easton Dam, WA

--- $\quad$ Gold Creek near Hyak, WA

--- $\quad$ Yakima River near Easton, WA

\section{Crab Creek Basin}

$47^{\circ} 45^{\prime} 24^{\prime \prime} \quad 118^{\circ} 42^{\prime} 11^{\prime \prime}$

$47^{\circ} 39^{\prime} 51^{\prime \prime} \quad 118^{\circ} 55^{\prime} 39^{\prime \prime}$

$47^{\circ} 24^{\prime} 24^{\prime \prime} \quad 118^{\circ} 19^{\prime} 07^{\prime \prime}$

charge

Discharge,

Water year 1977

Date in $\mathrm{ft}^{3} / \mathrm{s}$

10-02-2001

10-03-2001

10-09-2001

10-03-2001

$10-04-2001$

$10-09-2001$

$10-04-2001$

$10-03-2001$

$10-02-2001$

10-03-2001

10-04-2001

10-04-2001

10-02-2001

10-05-2001

10-10-2001

10-09-2001

9-19-2001

10-09-2001

10-09-2001

0.09

0.09
3.0
4.0

4.0

.10

31.0

.13
9.2

9.2
2.4

2.4

30.0

10.0

11.0

.23
8.5

9-20-1977

9-20-1977

Discharge,

10-04-2001

10-04-2001

10-04-2001

in $\mathrm{ft}^{3} / \mathrm{s}$

$47^{\circ} 39^{\prime} 46^{\prime \prime}$

$118^{\circ} 55^{\prime} 49^{\prime \prime}$

10-01-2001

10-01-2001

10-01-2001

10-01-2001

9-20-1977

akima River Basin

$46^{\circ} 13^{\prime} 02^{\prime \prime}$

$120^{\circ} 29^{\prime} 39^{\prime \prime}$

10-04-200

$46^{\circ} 28^{\prime} 49^{\prime \prime}$

$120^{\circ} 26^{\prime} 53^{\prime \prime}$

7-18-2001

46 $29^{\prime} 01^{\prime \prime} \quad 120^{\circ} 25^{\prime} 51^{\prime \prime}$

7-18-2001

$46^{\circ} 27^{\prime} 49^{\prime \prime} \quad 120^{\circ} 23^{\prime} 41^{\prime \prime} \quad 7-19-2001$

$46^{\circ} 24^{\prime} 25^{\prime \prime} \quad 120^{\circ} 18^{\prime} 36^{\prime \prime}$

$7-19-2001$

$46^{\circ} 37^{\prime} 13^{\prime \prime} \quad 121^{\circ} 18^{\prime} 05^{\prime \prime} \quad 10-02-200$

$47^{\circ} 24^{\prime} 54^{\prime \prime} \quad 121^{\circ} 04^{\prime} 53^{\prime \prime} \quad 10-03-200$

$47^{\circ} 24^{\prime} 01^{\prime \prime} \quad 121^{\circ} 05^{\prime} 44^{\prime \prime}$

$121^{\circ} 22^{\prime} 56^{\prime \prime} \quad 10-01-2001$

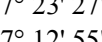

.09

.15

0

9-19-1977

9-19-1977

9-19-1977

9-19-1977

6.5

9-20-1977

119

2.5

.21
.26

0

2.0

8-23-1977

1.1

.13

8-23-1977

0

12.0

29.0

437

431
36.0

1.4

50.0

8-10-1977

8-10-1977

169

8-09-1977

.


Table 2. Low-flow measurement data for 410 selected sites in Washington, water years 1977 and 2001--Continued

\begin{tabular}{|c|c|c|c|c|c|c|c|}
\hline \multirow{2}{*}{$\begin{array}{l}\text { Gaging- } \\
\text { station } \\
\text { number }\end{array}$} & \multirow[b]{2}{*}{ Gaging-station name } & \multirow[b]{2}{*}{ Latitude } & \multirow[b]{2}{*}{ Longitude } & \multicolumn{2}{|c|}{ Water year 2001} & \multicolumn{2}{|c|}{ Water year 1977} \\
\hline & & & & Date & $\begin{array}{c}\text { Discharge, } \\
\text { in } \mathrm{ft}^{3} / \mathrm{s}\end{array}$ & Date & $\begin{array}{c}\text { Discharge, } \\
\text { in } \mathrm{ft}^{3} / \mathrm{s}\end{array}$ \\
\hline \multicolumn{8}{|c|}{ Yakima River Basin--Continued } \\
\hline--- & Big Creek near Easton, WA & $47^{\circ} 12^{\prime} 43^{\prime \prime}$ & $121^{\circ} 06^{\prime} 13^{\prime \prime}$ & $10-01-2001$ & 0.10 & $8-10-1977$ & 0.46 \\
\hline --- & Little Naches River near Cliffdell, WA & $46^{\circ} 59^{\prime} 16^{\prime \prime}$ & $121^{\circ} 05^{\prime} 54^{\prime \prime}$ & $9-18-2001$ & 10.0 & 8-16-1977 & 28.9 \\
\hline --- & Rattlesnake Creek near Nile, WA & $46^{\circ} 49^{\prime} 09^{\prime \prime}$ & $120^{\circ} 56^{\prime} 11^{\prime \prime}$ & $10-02-2001$ & 12.0 & 8-16-1977 & 11.7 \\
\hline --- & Manastash Creek near Naches, WA & $46^{\circ} 58^{\prime} 01^{\prime \prime}$ & $120^{\circ} 41^{\prime} 06^{\prime \prime}$ & 10-01-2001 & 4.9 & 8-11-1977 & 5.5 \\
\hline --- & Wenas Creek above Wenas Lake, WA & $46^{\circ} 50^{\prime} 13^{\prime \prime}$ & $120^{\circ} 43^{\prime} 06^{\prime \prime}$ & 10-04-2001 & 1.0 & 8-12-1977 & .80 \\
\hline 12474700 & Mosquito Creek near Easton, WA & $47^{\circ} 17^{\prime} 31^{\prime \prime}$ & $121^{\circ} 19^{\prime} 27^{\prime \prime}$ & $10-01-2001$ & .09 & 8-09-1977 & .16 \\
\hline 12475000 & Cabin Creek near Easton, WA & $47^{\circ} 14^{\prime} 22^{\prime \prime}$ & $121^{\circ} 13^{\prime} 38^{\prime \prime}$ & 10-01-2001 & 1.2 & 8-09-1977 & 8.2 \\
\hline 12480000 & Teanaway River Below Forks near Cle Elum, WA & $47^{\circ} 15^{\prime} 02^{\prime \prime}$ & $120^{\circ} 52^{\prime} 22^{\prime \prime}$ & $10-03-2001$ & 12.0 & & \\
\hline 12491700 & Hause Creek near Rimrock, WA & $46^{\circ} 40^{\prime} 32^{\prime \prime}$ & $121^{\circ} 04^{\prime} 53^{\prime \prime}$ & $10-02-2001$ & .01 & 8-12-1977 & 0 \\
\hline 12505000 & Yakima River near Parker, WA & $46^{\circ} 29^{\prime} 49^{\prime \prime}$ & $120^{\circ} 26^{\prime} 34^{\prime \prime}$ & $7-18-2001$ & 484 & & \\
\hline 12506300 & North Fork Simcoe Creek near Fort Simcoe, WA & $46^{\circ} 27^{\prime} 26^{\prime \prime}$ & $120^{\circ} 52^{\prime} 10^{\prime \prime}$ & $10-04-2001$ & 3.7 & 8-16-1977 & 2.6 \\
\hline 12506330 & South Fork Simcoe Creek near Fort Simcoe, WA & $46^{\circ} 26^{\prime} 40^{\prime \prime}$ & $120^{\circ} 53^{\prime} 13^{\prime \prime}$ & $10-04-2001$ & 2.7 & 8-16-1977 & .94 \\
\hline 12508480 & Dry Creek near Toppenish, WA & $46^{\circ} 15^{\prime} 14^{\prime \prime}$ & $120^{\circ} 24^{\prime} 13^{\prime \prime}$ & $10-04-2001$ & 0 & & \\
\hline \multicolumn{8}{|c|}{ Snake River Basin } \\
\hline --- & Pataha Creek near Pomeroy, WA & $46^{\circ} 21^{\prime} 39^{\prime \prime}$ & $117^{\circ} 33^{\prime} 24^{\prime \prime}$ & 9-20-2001 & .44 & & \\
\hline --- & Tucannon River near Dayton, WA & $46^{\circ} 14^{\prime} 35^{\prime \prime}$ & $117^{\circ} 41^{\prime} 18^{\prime \prime}$ & $9-21-2001$ & 40.0 & & \\
\hline--- & Pataha Creek near Starbuck, WA & $46^{\circ} 30^{\prime} 43^{\prime \prime}$ & $117^{\circ} 58^{\prime} 19^{\prime \prime}$ & $9-17-2001$ & 1.8 & & \\
\hline 13343510 & Alpowa Creek at Peola, WA & $46^{\circ} 19^{\prime} 02^{\prime \prime}$ & $117^{\circ} 29^{\prime} 31^{\prime \prime}$ & $9-20-2001$ & 0 & 8-09-1977 & 0 \\
\hline 13343680 & Deadman Creek near Central Ferry, WA & $46^{\circ} 37^{\prime} 07^{\prime \prime}$ & $117^{\circ} 45^{\prime} 09^{\prime \prime}$ & $9-20-2001$ & 3.0 & & \\
\hline 13343800 & Meadow Creek near Central Ferry, WA & $46^{\circ} 35^{\prime} 50^{\prime \prime}$ & $117^{\circ} 46^{\prime} 57^{\prime \prime}$ & $9-20-2001$ & 2.1 & 8-04-1977 & 1.2 \\
\hline 13344000 & Tucannon River near Pomeroy, WA & $46^{\circ} 26^{\prime} 24^{\prime \prime}$ & $117^{\circ} 44^{\prime} 59^{\prime \prime}$ & $9-20-2001$ & 45.0 & & \\
\hline 13349400 & Pine Creek at Pine City, WA & $47^{\circ} 12^{\prime} 23^{\prime \prime}$ & $117^{\circ} 30^{\prime} 17^{\prime \prime}$ & $10-09-2001$ & 2.6 & & \\
\hline 13350500 & Union Flat Creek near Colfax, WA & $46^{\circ} 48^{\prime} 36^{\prime \prime}$ & $117^{\circ} 25^{\prime} 55^{\prime \prime}$ & $10-09-2001$ & 1.6 & & \\
\hline 13352500 & Cow Creek at Hooper, WA & $46^{\circ} 45^{\prime} 45^{\prime \prime}$ & $118^{\circ} 08^{\prime} 49^{\prime \prime}$ & $10-05-2001$ & 2.9 & & \\
\hline \multicolumn{8}{|c|}{ Walla Walla River Basin } \\
\hline--- & South Fork Touchet River at Dayton, WA & $46^{\circ} 18^{\prime} 03^{\prime \prime}$ & $117^{\circ} 57^{\prime} 33^{\prime \prime}$ & $9-21-2001$ & 1.3 & $8-10-1977$ & .75 \\
\hline--- & Walla Walla River near College Place, WA & $46^{\circ} 01^{\prime} 00^{\prime \prime}$ & $118^{\circ} 23^{\prime} 57^{\prime \prime}$ & $9-13-2001$ & 14.0 & $8-01-1977$ & 3.6 \\
\hline--- & East Little Walla Walla River near State Line, WA & $46^{\circ} 00^{\prime} 45^{\prime \prime}$ & $118^{\circ} 24^{\prime} 42^{\prime \prime}$ & $9-12-2001$ & 12.0 & $8-11-1977$ & 3.1 \\
\hline--- & Mill Creek near Walla Walla, WA & $45^{\circ} 59^{\prime} 23^{\prime \prime}$ & $118^{\circ} 03^{\prime} 01^{\prime \prime}$ & $9-05-2001$ & 14.0 & & \\
\hline--- & Mud Creek near Lowden, WA & $46^{\circ} 02^{\prime} 31^{\prime \prime}$ & $118^{\circ} 36^{\prime} 52^{\prime \prime}$ & $9-07-2001$ & 1.3 & $8-12-1977$ & .54 \\
\hline--- & Wolf Creek near Dayton, WA & $46^{\circ} 14^{\prime} 17^{\prime \prime}$ & $117^{\circ} 53^{\prime} 45^{\prime \prime}$ & $9-21-2001$ & 23.0 & & \\
\hline--- & Mill Creek near Whitman Mission, WA & $46^{\circ} 02^{\prime} 30^{\prime \prime}$ & $118^{\circ} 28^{\prime} 14^{\prime \prime}$ & $10-04-2001$ & 3.6 & & \\
\hline--- & Russell Creek near Langdon, WA & $46^{\circ} 01^{\prime} 45^{\prime \prime}$ & $118^{\circ} 20^{\prime} 40^{\prime \prime}$ & $10-04-2001$ & 0 & & \\
\hline--- & Cottonwood Creek near Langdon, WA & $46^{\circ} 01^{\prime} 32^{\prime \prime}$ & $118^{\circ} 20^{\prime} 45^{\prime \prime}$ & $10-04-2001$ & 0 & $8-11-1977$ & .04 \\
\hline 14013500 & Blue Creek near Walla Walla, WA & $46^{\circ} 03^{\prime} 27^{\prime \prime}$ & $118^{\circ} 08^{\prime} 24^{\prime \prime}$ & $9-05-2001$ & .65 & & \\
\hline 14013600 & Mill Creek below Blue Creek near Walla Walla, WA & $46^{\circ} 04^{\prime} 52^{\prime \prime}$ & $118^{\circ} 11^{\prime} 23^{\prime \prime}$ & $9-05-2001$ & 29.0 & & \\
\hline 14014400 & Yellowhawk Creek near College Place, WA & $46^{\circ} 01^{\prime} 08^{\prime \prime}$ & $118^{\circ} 23^{\prime} 57^{\prime \prime}$ & $9-13-2001$ & 13.0 & $8-11-1977$ & 7.0 \\
\hline 14016000 & Dry Creek near Walla Walla, WA & $46^{\circ} 07^{\prime} 19^{\prime \prime}$ & $118^{\circ} 14^{\prime} 13^{\prime \prime}$ & $9-14-2001$ & .80 & & \\
\hline 14016050 & Dry Creek at Lowden,WA & $46^{\circ} 03^{\prime} 24^{\prime \prime}$ & $118^{\circ} 35^{\prime} 26^{\prime \prime}$ & $9-07-2001$ & .98 & $8-12-1977$ & .25 \\
\hline 14016500 & East Fork Touchet River near Dayton, WA & $46^{\circ} 16^{\prime} 45^{\prime \prime}$ & $117^{\circ} 54^{\prime} 07^{\prime \prime}$ & $9-21-2001$ & 38.0 & & \\
\hline
\end{tabular}


Table 2. Low-flow measurement data for 410 selected sites in Washington, water years 1977 and 2001--Continued

\begin{tabular}{cl}
$\begin{array}{c}\text { Gaging- } \\
\text { station } \\
\text { number }\end{array}$ & \multicolumn{1}{c}{ Gaging- } \\
& \\
14016800 & Patit Creek near Dayton,WA \\
14016950 & Coppei Creek near Waitsburg,WA \\
14017000 & Touchet River at Bolles, WA \\
14017070 & East Fork McKay Creek near Hunt \\
& \\
--- & Rock Creek near Goldendale, WA \\
--- & Wood Gulch near Roosevelt, WA \\
--- & Glade Creek near Patterson, WA \\
14034325 & Alder Creek near Bickleton, WA \\
14036600 & Rock Creek near Roosevelt, WA
\end{tabular}

--- $\quad$ Outlet Creek near Glenwood, WA

--- McCreedy Creek near Glenwood, WA

--- $\quad$ Summit Creek near Glenwood, WA

14106500 Pearl Creek near Glenwood, WA

14108500 Cunningham Creek near Glenwood, WA

14112000 Little Klickitat River near Goldendale, WA

--- Whipple Creek near Ridgefield, WA

--- $\quad$ Rock Creek at mouth at Stevenson, WA

Fifth Plain Creek near Proebstel, WA

Salmon Creek near Felida, WA

Curtin Creek near Felida, WA

Mill Creek near Felida, WA

Panther Creek near Carson, WA

Salmon Creek near Orchards, WA

Gee Creek near Ridgefield, WA

Weaver Creek near Orchards, WA

Burnt Bridge Creek at Vancouver, WA

Washougal River near Washougal, WA

Campen Creek near Camas, WA

Gibbons Creek near Camas, WA

Canyon Creek near Prindle, WA

Duncan Creek at Skamania, WA

Woodward Creek near Skamania, WA

Cougar Creek near Washougal, WA

Hamilton Creek near North Bonneville, WA

Wildboy Creek near Prindle, WA

Stebbins Creek near Washougal, WA

Greenleaf Creek near North Bonneville, WA

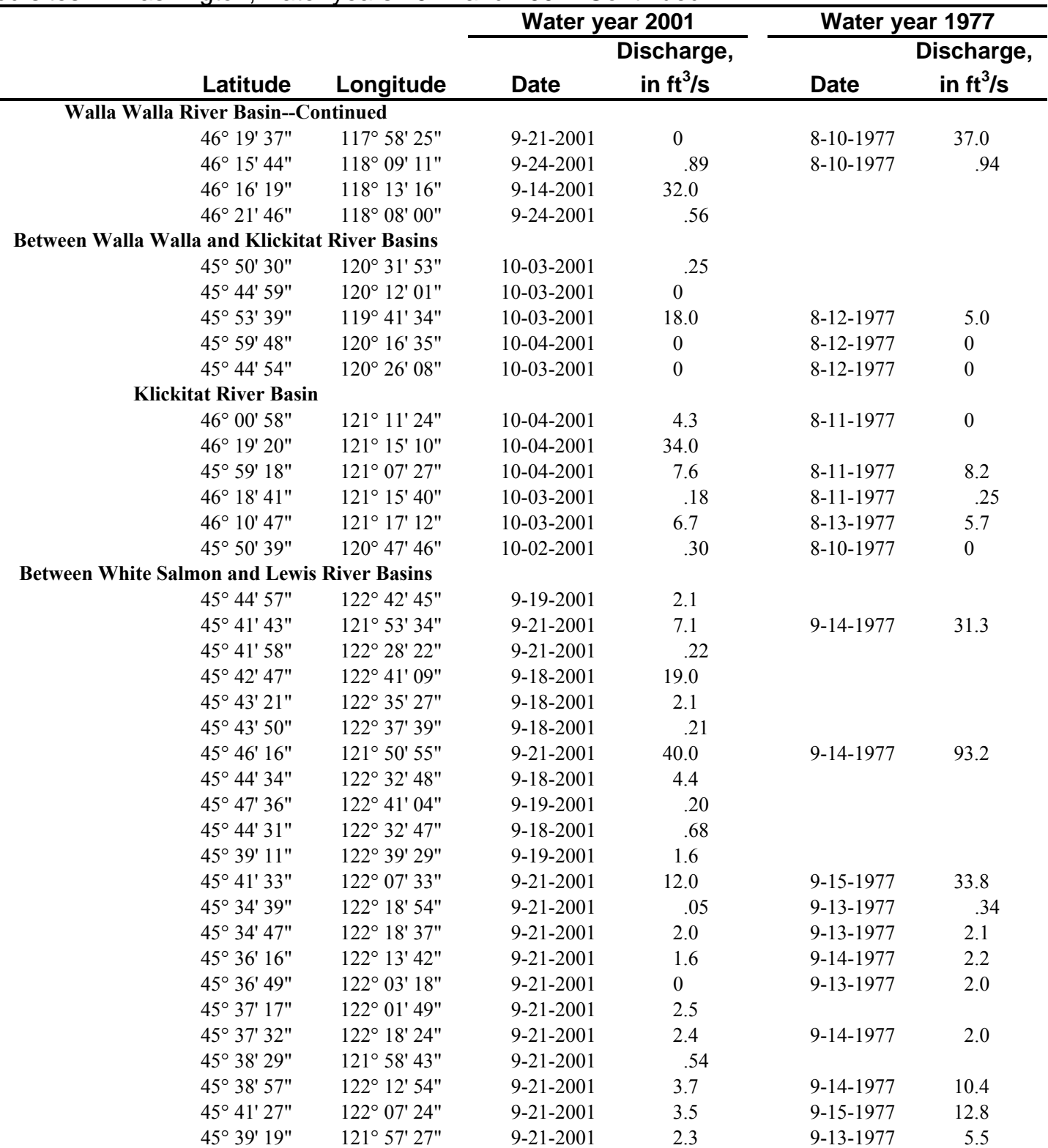


Table 2. Low-flow measurement data for 410 selected sites in Washington, water years 1977 and 2001--Continued

\begin{tabular}{|c|c|c|c|c|c|c|c|}
\hline \multirow{2}{*}{$\begin{array}{l}\text { Gaging- } \\
\text { station } \\
\text { number }\end{array}$} & \multirow[b]{2}{*}{ Gaging-station name } & & & \multicolumn{2}{|c|}{ Water year 2001} & \multicolumn{2}{|c|}{ Water year 1977} \\
\hline & & Latitude & Longitude & Date & $\begin{array}{c}\text { Discharge, } \\
\text { in } \mathrm{ft}^{3} / \mathrm{s}\end{array}$ & Date & $\begin{array}{c}\text { Discharge, } \\
\text { in } \mathrm{ft}^{3} / \mathrm{s}\end{array}$ \\
\hline \multicolumn{8}{|c|}{ Between White Salmon and Lewis River Basins--Continued } \\
\hline--- & Lawton Creek near Camas, WA & $45^{\circ} 33^{\prime} 43^{\prime \prime}$ & $122^{\circ} 15^{\prime} 59^{\prime \prime}$ & $9-21-2001$ & 1.6 & 9-13-1977 & 1.5 \\
\hline--- & Matney Creek near Camas, WA & $45^{\circ} 40^{\prime} 03^{\prime \prime}$ & $122^{\circ} 26^{\prime} 09^{\prime \prime}$ & 9-24-2001 & .33 & & \\
\hline--- & Dougan Creek near Washougal, WA & $45^{\circ} 40^{\prime} 27^{\prime \prime}$ & $122^{\circ} 09^{\prime} 23^{\prime \prime}$ & $9-21-2001$ & 2.2 & $9-15-1977$ & 8.9 \\
\hline--- & Little Washougal River near Washougal, WA & $45^{\circ} 38^{\prime} 46^{\prime \prime}$ & $122^{\circ} 21^{\prime} 24^{\prime \prime}$ & 9-24-2001 & 6.0 & & \\
\hline 14124000 & Little White Salmon River near Willard, WA & $45^{\circ} 47^{\prime} 55^{\prime \prime}$ & $121^{\circ} 38^{\prime} 24^{\prime \prime}$ & $9-21-2001$ & 23.0 & $8-17-1977$ & 7.2 \\
\hline 14143000 & West Fork Washougal River near Washougal, WA & $45^{\circ} 36^{\prime} 57^{\prime \prime}$ & $122^{\circ} 13^{\prime} 10^{\prime \prime}$ & $9-20-2001$ & 20.0 & & \\
\hline 14143500 & Washougal River near Washougal, WA & $45^{\circ} 37^{\prime} 22^{\prime \prime}$ & $122^{\circ} 17^{\prime} 39^{\prime \prime}$ & $9-19-2001$ & 59.0 & & \\
\hline 14144000 & Little Washougal River near Washougal, WA & $45^{\circ} 36^{\prime} 50^{\prime \prime}$ & $122^{\circ} 21^{\prime} 30^{\prime \prime}$ & $9-21-2001$ & 8.1 & $9-14-1977$ & 15.2 \\
\hline 14144500 & Lacamas Creek at Proebstel, WA & $45^{\circ} 40^{\prime} 18^{\prime \prime}$ & $122^{\circ} 29^{\prime} 17^{\prime \prime}$ & $9-21-2001$ & 3.0 & $9-15-1977$ & 5.5 \\
\hline 14144560 & Fifth Plain Creek at Highway 500 near Proebstel, WA & $45^{\circ} 40^{\prime} 20^{\prime \prime}$ & $122^{\circ} 29^{\prime} 39^{\prime \prime}$ & $9-21-2001$ & 1.7 & $9-15-1977$ & 3.2 \\
\hline 14211898 & Burnt Bridge Creek at 18th Street at Vancouver, WA & $45^{\circ} 38^{\prime} 04^{\prime \prime}$ & $122^{\circ} 37^{\prime} 25^{\prime \prime}$ & $9-25-2001$ & 1.2 & & \\
\hline 14211901 & Cold Creek at mouth at Vancouver, WA & $45^{\circ} 39^{\prime} 41^{\prime \prime}$ & $122^{\circ} 40^{\prime} 03^{\prime \prime}$ & $9-25-2001$ & .44 & & \\
\hline 14211902 & Burnt Bridge Creek near mouth at Vancouver, WA & $45^{\circ} 39^{\prime} 40^{\prime \prime}$ & $122^{\circ} 40^{\prime} 08^{\prime \prime}$ & $9-25-2001$ & 2.4 & & \\
\hline 14212000 & Salmon Creek near Battle Ground, WA & $45^{\circ} 46^{\prime} 25^{\prime \prime}$ & $122^{\circ} 26^{\prime} 44^{\prime \prime}$ & $9-18-2001$ & 2.9 & $9-16-1977$ & 4.9 \\
\hline \multicolumn{8}{|c|}{ Kalama River Basin } \\
\hline--- & Hatchery Creek near Kalama, WA & $46^{\circ} 02^{\prime} 42^{\prime \prime}$ & $122^{\circ} 48^{\prime} 15^{\prime \prime}$ & 9-14-2001 & 9.0 & $9-14-1977$ & 14.0 \\
\hline--- & Wild Horse Creek near Pigeon Springs, WA & $46^{\circ} 02^{\prime} 53^{\prime \prime}$ & $122^{\circ} 38^{\prime} 02^{\prime \prime}$ & $9-15-2001$ & 3.6 & $9-14-1977$ & 7.8 \\
\hline--- & Italian Creek near Pigeon Springs, WA & $46^{\circ} 02^{\prime} 00^{\prime \prime}$ & $122^{\circ} 43^{\prime} 46^{\prime \prime}$ & $9-15-2001$ & .66 & $9-14-1977$ & .92 \\
\hline--- & Gobar Creek at Pigeon Springs, WA & $46^{\circ} 03^{\prime} 06^{\prime \prime}$ & $122^{\circ} 37^{\prime} 26^{\prime \prime}$ & $9-15-2001$ & 12.0 & $9-14-1977$ & 15.4 \\
\hline 14223500 & Kalama River Below Italian Creek near Kalama, WA & $46^{\circ} 02^{\prime} 41^{\prime \prime}$ & $122^{\circ} 48^{\prime} 55^{\prime \prime}$ & 9-14-2001 & 189 & $9-15-1977$ & 269 \\
\hline \multicolumn{8}{|c|}{ Between Kalama and Cowlitz River Basins } \\
\hline--- & Owl Creek near Carrolls, WA & $46^{\circ} 05^{\prime} 29^{\prime \prime}$ & $122^{\circ} 52^{\prime} 09^{\prime \prime}$ & 9-14-2001 & .21 & $9-15-1977$ & .51 \\
\hline \multicolumn{8}{|c|}{ Cowlitz River Basin } \\
\hline--- & Tilton River near Morton, WA & $46^{\circ} 36^{\prime} 09^{\prime \prime}$ & $122^{\circ} 14^{\prime} 02^{\prime \prime}$ & $9-17-2001$ & 5.7 & & \\
\hline 14235500 & West Fork Tilton River near Morton, WA & $46^{\circ} 36^{\prime} 39^{\prime \prime}$ & $122^{\circ} 14^{\prime} 40^{\prime \prime}$ & $9-17-2001$ & 6.8 & & \\
\hline 14237500 & Winston Creek near Silver Lake, WA & $46^{\circ} 28^{\prime} 56^{\prime \prime}$ & $122^{\circ} 31^{\prime} 17^{\prime \prime}$ & $9-17-2001$ & 5.4 & & \\
\hline 14239000 & Salmon Creek near Toledo, WA & $46^{\circ} 24^{\prime} 49^{\prime \prime}$ & $122^{\circ} 49^{\prime} 19^{\prime \prime}$ & $9-15-2001$ & 1.3 & & \\
\hline 14245000 & Coweman River near Kelso, WA & $46^{\circ} 07^{\prime} 41^{\prime \prime}$ & $122^{\circ} 50^{\prime} 18^{\prime \prime}$ & $9-22-2001$ & 36.0 & & \\
\hline \multicolumn{8}{|c|}{ Lower Columbia River Basin } \\
\hline--- & Fall Creek near Grays River, WA & $46^{\circ} 22^{\prime} 24^{\prime \prime}$ & $123^{\circ} 35^{\prime} 24^{\prime \prime}$ & 9-20-2001 & 1.5 & & \\
\hline--- & Fossil Creek near Grays River, WA & $46^{\circ} 21^{\prime} 43^{\prime \prime}$ & $123^{\circ} 33^{\prime} 14^{\prime \prime}$ & 9-19-2001 & 2.6 & 9-14-1977 & 9.3 \\
\hline--- & Klints Creek near Grays River, WA & $46^{\circ} 21^{\prime} 21^{\prime \prime}$ & $123^{\circ} 33^{\prime} 34^{\prime \prime}$ & 9-19-2001 & 1.2 & 9-14-1977 & 4.5 \\
\hline--- & Malone Creek at Rosbury, WA & $46^{\circ} 20^{\prime} 07^{\prime \prime}$ & $123^{\circ} 38^{\prime} 36^{\prime \prime}$ & $9-20-2001$ & 1.3 & $9-14-1977$ & 3.9 \\
\hline--- & West Fork Elochoman River near Skamokawa, WA & $46^{\circ} 19^{\prime} 19^{\prime \prime}$ & $123^{\circ} 15^{\prime} 46^{\prime \prime}$ & $9-18-2001$ & 8.2 & $9-13-1977$ & 19.2 \\
\hline--- & Skamokawa Creek near Skamokawa, WA & $46^{\circ} 19^{\prime} 10^{\prime \prime}$ & $123^{\circ} 27^{\prime} 20^{\prime \prime}$ & 9-18-2001 & 8.2 & 9-15-1977 & 30.1 \\
\hline--- & Deep River near Rosbury, WA & $46^{\circ} 21^{\prime} 37^{\prime \prime}$ & $123^{\circ} 40^{\prime} 59^{\prime \prime}$ & $9-20-2001$ & .88 & $9-14-1977$ & 2.1 \\
\hline--- & Falk Creek near Skamokawa, WA & $46^{\circ} 17^{\prime} 57^{\prime \prime}$ & $123^{\circ} 26^{\prime} 24^{\prime \prime}$ & 9-19-2001 & 1.0 & $9-14-1977$ & 4.8 \\
\hline--- & Clark Creek near Longview, WA & $46^{\circ} 10^{\prime} 45^{\prime \prime}$ & $122^{\circ} 59^{\prime} 35^{\prime \prime}$ & 9-14-2001 & .27 & 9-15-1977 & .78 \\
\hline--- & Crooked Creek near Rosbury, WA & $46^{\circ} 17^{\prime} 55^{\prime \prime}$ & $123^{\circ} 38^{\prime} 56^{\prime \prime}$ & 9-19-2001 & 1.6 & & \\
\hline--- & Wilson Creek near Skamokawa, WA & $46^{\circ} 17^{\prime} 19^{\prime \prime}$ & $123^{\circ} 26^{\prime} 19^{\prime \prime}$ & 9-19-2001 & 8.7 & 9-14-1977 & 21.9 \\
\hline
\end{tabular}


Table 2. Low-flow measurement data for 410 selected sites in Washington, water years 1977 and 2001--Continued

\begin{tabular}{cl}
$\begin{array}{c}\text { Gaging- } \\
\text { station } \\
\text { number }\end{array}$ & \multicolumn{1}{c}{ Gaging-station name } \\
\hline & \\
--- & Abernathy Creek near Longview, WA \\
--- & Beaver Creek near Cathlamet, WA \\
--- & Slide Creek near Longview, WA \\
--- & Harmony Creek near Stella, WA \\
--- & Coal Creek near Stella, WA \\
--- & Elochoman River near Skamokawa, WA \\
14246000 & Abernathy Creek near Longview, WA \\
14247500 & Elochoman River near Cathlamet, WA \\
14248000 & Skamokawa Creek near Skamokawa, WA \\
14249000 & Grays River above South Fork near Grays River, WA \\
14250900 & Grays River near Grays River, WA
\end{tabular}

\begin{tabular}{|c|c|c|}
\hline & Latitude & Longitude \\
\hline \multicolumn{3}{|c|}{ Lower Columbia River Basin--Continued } \\
\hline & $46^{\circ} 15^{\prime} 37^{\prime \prime}$ & $123^{\circ} 10^{\prime} 36^{\prime \prime}$ \\
\hline & $46^{\circ} 13^{\prime} 33^{\prime \prime}$ & $123^{\circ} 19^{\prime} 14^{\prime \prime}$ \\
\hline & $46^{\circ} 12^{\prime} 31^{\prime \prime}$ & $123^{\circ} 09^{\prime} 08^{\prime \prime}$ \\
\hline & $46^{\circ} 11^{\prime} 15^{\prime \prime}$ & $123^{\circ} 02^{\prime} 10^{\prime \prime}$ \\
\hline & $46^{\circ} 11^{\prime} 15^{\prime \prime}$ & $123^{\circ} 02^{\prime} 04^{\prime \prime}$ \\
\hline & $46^{\circ} 19^{\prime} 10^{\prime \prime}$ & $123^{\circ} 15^{\prime} 27^{\prime \prime}$ \\
\hline & $46^{\circ} 12^{\prime} 09^{\prime \prime}$ & $123^{\circ} 09^{\prime} 19^{\prime \prime}$ \\
\hline & $46^{\circ} 13^{\prime} 16^{\prime \prime}$ & $123^{\circ} 20^{\prime} 32^{\prime \prime}$ \\
\hline & $46^{\circ} 17^{\prime} 56^{\prime \prime}$ & $123^{\circ} 26^{\prime} 38^{\prime \prime}$ \\
\hline & $46^{\circ} 23^{\prime} 35^{\prime \prime}$ & $123^{\circ} 28^{\prime} 43^{\prime \prime}$ \\
\hline & $46^{\circ} 21^{\prime} 33^{\prime \prime}$ & $123^{\circ} 33^{\prime} 59^{\prime \prime}$ \\
\hline
\end{tabular}

Water year 2001

\begin{tabular}{|c|c|c|c|}
\hline \multicolumn{2}{|c|}{ Water year 2001} & \multicolumn{2}{|c|}{ Water year 1977} \\
\hline Date & $\begin{array}{c}\text { Discharge, } \\
\text { in } \mathrm{ft}^{3} / \mathrm{s}\end{array}$ & Date & $\begin{array}{c}\text { Discharge, } \\
\text { in } \mathrm{ft}^{3} / \mathrm{s}\end{array}$ \\
\hline $9-17-2001$ & 6.2 & $9-15-1977$ & 14.3 \\
\hline $9-18-2001$ & 1.9 & $9-13-1977$ & 5.2 \\
\hline $9-17-2001$ & .50 & $9-15-1977$ & 1.1 \\
\hline $9-14-2001$ & .89 & $9-15-1977$ & 1.8 \\
\hline $9-14-2001$ & 4.2 & $9-15-1977$ & 9.3 \\
\hline $9-18-2001$ & 23.0 & & \\
\hline $9-17-2001$ & 10.0 & $9-15-1977$ & 20.3 \\
\hline $9-18-2001$ & 37.0 & & \\
\hline $9-18-2001$ & 10.0 & 9-14-1977 & 31.0 \\
\hline $9-19-2001$ & 26.0 & $9-15-1977$ & 73.9 \\
\hline $9-20-2001$ & 62.0 & $8-12-1977$ & 39.4 \\
\hline
\end{tabular}


Table 3 
Table 3. Low-flow measurement data for 166 selected sites in Oregon, water years 1977 and 2001

[latitude and longitude are based on the North American Datum of 1983 and shown in degrees, minutes, seconds; $\mathrm{ft}^{3} \mathrm{~s}$, cubic feet per second; OR, Oregon; ---, no number assigned]

\begin{tabular}{|c|c|c|c|c|c|c|c|}
\hline \multirow{2}{*}{$\begin{array}{l}\text { Gaging- } \\
\text { station } \\
\text { number }\end{array}$} & \multirow[b]{2}{*}{ Gaging-station name } & \multirow[b]{2}{*}{ Latitude } & \multirow[b]{2}{*}{ Longitude } & \multicolumn{2}{|c|}{ Water year 2001} & \multicolumn{2}{|c|}{ Water year 1977} \\
\hline & & & & Date & $\begin{array}{c}\text { Discharge, } \\
\text { in } \mathrm{ft}^{3} / \mathrm{s}\end{array}$ & Date & $\begin{array}{c}\text { Discharge, } \\
\text { in } \mathrm{ft}^{3} / \mathrm{s}\end{array}$ \\
\hline \multicolumn{8}{|c|}{ Warner Lakes Basin } \\
\hline 10371500 & Deep Creek above Adel, OR & $42^{\circ} 11^{\prime} 21^{\prime \prime}$ & $120^{\circ} 00^{\prime} 06^{\prime \prime}$ & $9-05-2001$ & 6.4 & 8-01-1977 & 3.7 \\
\hline 10378500 & Honey Creek near Plush, OR & $42^{\circ} 27^{\prime} 00^{\prime \prime}$ & $120^{\circ} 04^{\prime} 04^{\prime \prime}$ & $8-15-2001$ & .28 & 8-24-1977 & .25 \\
\hline \multicolumn{8}{|c|}{ Albert Lake Basin } \\
\hline 10384000 & Chewaucan River near Paisley, OR & $42^{\circ} 41^{\prime} 04^{\prime \prime}$ & $120^{\circ} 34^{\prime} 12^{\prime \prime}$ & $9-05-2001$ & 16.5 & $7-29-1977$ & 9.6 \\
\hline 10388001 & Ana River plus canal near Summer Lake, OR & $42^{\circ} 59^{\prime} 42^{\prime \prime}$ & $120^{\circ} 44^{\prime} 58^{\prime \prime}$ & $9-12-2001$ & 14.7 & 7-10-1977 & 75.0 \\
\hline \multicolumn{8}{|c|}{ Summer Lake Basin } \\
\hline \multicolumn{8}{|c|}{ Goose Lake Basin } \\
\hline 11340500 & \multicolumn{6}{|c|}{ Klamath River Basin } & 0 \\
\hline 11497500 & Sprague near Beatty, OR & $42^{\circ} 26^{\prime} 49^{\prime \prime}$ & $121^{\circ} 14^{\prime} 19^{\prime \prime}$ & $9-14-2001$ & 107 & 8-11-1977 & 57.0 \\
\hline 11499000 & Sycan River near Beatty, OR & $42^{\circ} 33^{\prime} 00^{\prime \prime}$ & $121^{\circ} 19^{\prime} 04^{\prime \prime}$ & $9-14-2001$ & 18.7 & 9-07-1977 & 7.8 \\
\hline 11503100 & Munson Creek near Rim Village, OR & $42^{\circ} 52^{\prime} 45^{\prime \prime}$ & $122^{\circ} 08^{\prime} 19^{\prime \prime}$ & $9-12-2001$ & 2.0 & $8-12-1977$ & .08 \\
\hline--- & Sun Creek at Sun Meadow, OR & $42^{\circ} 45^{\prime} 44^{\prime \prime}$ & $122^{\circ} 00^{\prime} 57^{\prime \prime}$ & $9-12-2001$ & 7.0 & 8-11-1977 & 8.66 \\
\hline--- & Bear Creek at Crater Lake Park Boundary, OR & $42^{\circ} 58^{\prime} 50^{\prime \prime}$ & $121^{\circ} 58^{\prime} 39^{\prime \prime}$ & $9-13-2001$ & 0 & 8-11-1977 & 0 \\
\hline--- & Spring Creek at Collier State Park, OR & $42^{\circ} 38^{\prime} 38^{\prime \prime}$ & $121^{\circ} 52^{\prime} 48^{\prime \prime}$ & $9-13-2001$ & 281 & 8-23-1977 & 293 \\
\hline--- & Wood River Springs above Wood River, OR & $42^{\circ} 44^{\prime} 03^{\prime \prime}$ & $121^{\circ} 59^{\prime} 18^{\prime \prime}$ & $9-13-2001$ & 18.8 & & \\
\hline 11504000 & Wood River at Fort Klamath, OR & $42^{\circ} 41^{\prime} 59^{\prime \prime}$ & $121^{\circ} 59^{\prime} 04^{\prime \prime}$ & $9-13-2001$ & 132 & 8-26-1977 & 131 \\
\hline 11504040 & Fort Creek near Fort Klamath, OR & $42^{\circ} 40^{\prime} 53^{\prime \prime}$ & $121^{\circ} 58^{\prime} 32^{\prime \prime}$ & $9-13-2001$ & 52.5 & 8-26-1977 & 65.4 \\
\hline 11510000 & Spencer Creek near Keno, OR & $42^{\circ} 09^{\prime} 29^{\prime \prime}$ & $122^{\circ} 01^{\prime} 44^{\prime \prime}$ & $9-13-2001$ & 2.2 & $8-22-1977$ & 6.2 \\
\hline \multicolumn{8}{|c|}{ Owyhee River Basin } \\
\hline 13177985 & Jordan Creek at DeLamar Mine near Jordan Valley, OR & $43^{\circ} 01^{\prime} 26^{\prime \prime}$ & $116^{\circ} 51^{\prime} 19^{\prime \prime}$ & $9-11-2001$ & 0 & & \\
\hline 13181500 & Crooked Creek near Rome, OR & $42^{\circ} 48^{\prime} 00^{\prime \prime}$ & $117^{\circ} 44^{\prime} 03^{\prime \prime}$ & $9-11-2001$ & 21.8 & 9-09-1977 & 22.9 \\
\hline \multicolumn{8}{|c|}{ Malheur River Basin } \\
\hline 13214000 & Malheur River near Drewsey, OR & $43^{\circ} 47^{\prime} 04^{\prime \prime}$ & $118^{\circ} 19^{\prime} 54^{\prime \prime}$ & $9-13-2001$ & 1.8 & 9-06-1977 & .73 \\
\hline 13216500 & North Fork Malheur River above Beulah Reservoir, OR & $43^{\circ} 56^{\prime} 53^{\prime \prime}$ & $118^{\circ} 10^{\prime} 28^{\prime \prime}$ & $9-24-2001$ & 38.0 & 8-10-1977 & 24.0 \\
\hline \multicolumn{8}{|c|}{ Burnt River Basin } \\
\hline 13270800 & \multicolumn{6}{|c|}{ Imnaha River Basin } & 19.0 \\
\hline 13291000 & Imnaha River above Gumboot Creek, OR & $45^{\circ} 10^{\prime} 59^{\prime \prime}$ & $116^{\circ} 52^{\prime} 04^{\prime \prime}$ & 9-07-2001 & 62.0 & 8-08-1977 & 53.4 \\
\hline--- & Little Sheep Creek near Imnaha, OR & $45^{\circ} 30^{\prime} 49^{\prime \prime}$ & $116^{\circ} 51^{\prime} 46^{\prime \prime}$ & $9-06-2001$ & 12.0 & 8-11-1977 & 11.2 \\
\hline \multicolumn{8}{|c|}{ Grande Ronde River Basin } \\
\hline--- & Chesnismus Creek near Lewis, OR & $45^{\circ} 42^{\prime} 50^{\prime \prime}$ & $117^{\circ} 09^{\prime} 15^{\prime \prime}$ & $10-11-2001$ & 7.5 & 8-10-1977 & 2.08 \\
\hline 13320000 & Catherine Creek near Union, OR & $45^{\circ} 09^{\prime} 20^{\prime \prime}$ & $117^{\circ} 46^{\prime} 30^{\prime \prime}$ & $9-24-2001$ & 17.5 & $8-21-1977$ & 16.0 \\
\hline 13323600 & Indian Creek near Imbler, OR & $45^{\circ} 25^{\prime} 59^{\prime \prime}$ & $117^{\circ} 49^{\prime} 24^{\prime \prime}$ & $10-11-2001$ & 14.0 & 8-19-1977 & 3.32 \\
\hline 13324300 & Lookingglass Creek at Palmer Junction, OR & $45^{\circ} 42^{\prime} 27^{\prime \prime}$ & $117^{\circ} 50^{\prime} 34^{\prime \prime}$ & $10-10-2001$ & 52.0 & 8-11-1977 & 51.3 \\
\hline 13333000 & Wenaha River at Troy, OR & $45^{\circ} 56^{\prime} 44^{\prime \prime}$ & $117^{\circ} 27^{\prime} 07^{\prime \prime}$ & $9-18-2001$ & 144 & 8-10-1977 & 141 \\
\hline
\end{tabular}


Table 3. Low-flow measurement data for 166 selected sites in Oregon, water years 1977 and 2001--Continued

\begin{tabular}{|c|c|}
\hline $\begin{array}{c}\text { Gaging- } \\
\text { station } \\
\text { number }\end{array}$ & Gaging-station name \\
\hline--- & Pine Creek near Umapine, OR \\
\hline--- & Dry Creek near Barrett, OR \\
\hline--- & Birch Creek near Spofford, OR \\
\hline--- & Line Creek near Conway, OR \\
\hline 14022500 & McKay Creek near Pilot Rock, OR \\
\hline--- & Boston Creek near Gibbon, OR \\
\hline--- & Umatilla River near Thorn Hollow, OR \\
\hline--- & Eagle Creek near Athena, OR \\
\hline 14034800 & Rhea Creek near Heppner, OR \\
\hline--- & South Fork John Day River near Poison Creek near Izee, OR \\
\hline --- & Deer Creek near McMullen Spring near Dayville, OR \\
\hline --- & Tex Creek near mouth near Mount Vernon, OR \\
\hline--- & Murderers Creek at mouth near Dayville, OR \\
\hline--- & Black Canyon Creek above Black Canyon near Dayville, OR \\
\hline 14038530 & John Day River near John Day, OR \\
\hline--- & Desolation Creek above Dale, OR \\
\hline 14041000 & Desolation Creek near Dale, OR \\
\hline 14041500 & North Fork John Day River near Dale, OR \\
\hline--- & North Fork John Day River above Oriental, OR \\
\hline 14042000 & Camas Creek near Lehman, OR \\
\hline 14043000 & Cable Creek near Ukiah, OR \\
\hline--- & Wall Creek near Monument, OR \\
\hline 14047380 & Lone Rock Creek near Lone Rock, OR \\
\hline 14047390 & Rock Creek near Condon, OR \\
\hline 14050000 & Deschutes River below Snow Creek near La Pine, OR \\
\hline 14052000 & Deer Creek above Crane near La Pine, OR \\
\hline 14052500 & Quinn River near La Pine, OR \\
\hline 14054500 & Brown Creek near La Pine, OR \\
\hline 14057500 & Fall River near La Pine, OR \\
\hline 14073000 & Tumalo Creek near Bend, OR \\
\hline 14079500 & Crooked River near Post, OR \\
\hline 14080250 & Bear Creek near Prineville, OR \\
\hline--- & Trout Creek near Gateway at Highway 97, OR \\
\hline 14097200 & White River near Government Camp, OR \\
\hline 14099000 & White River near Wapinitia, OR \\
\hline--- & Badger Creek near Warm Springs, OR \\
\hline
\end{tabular}

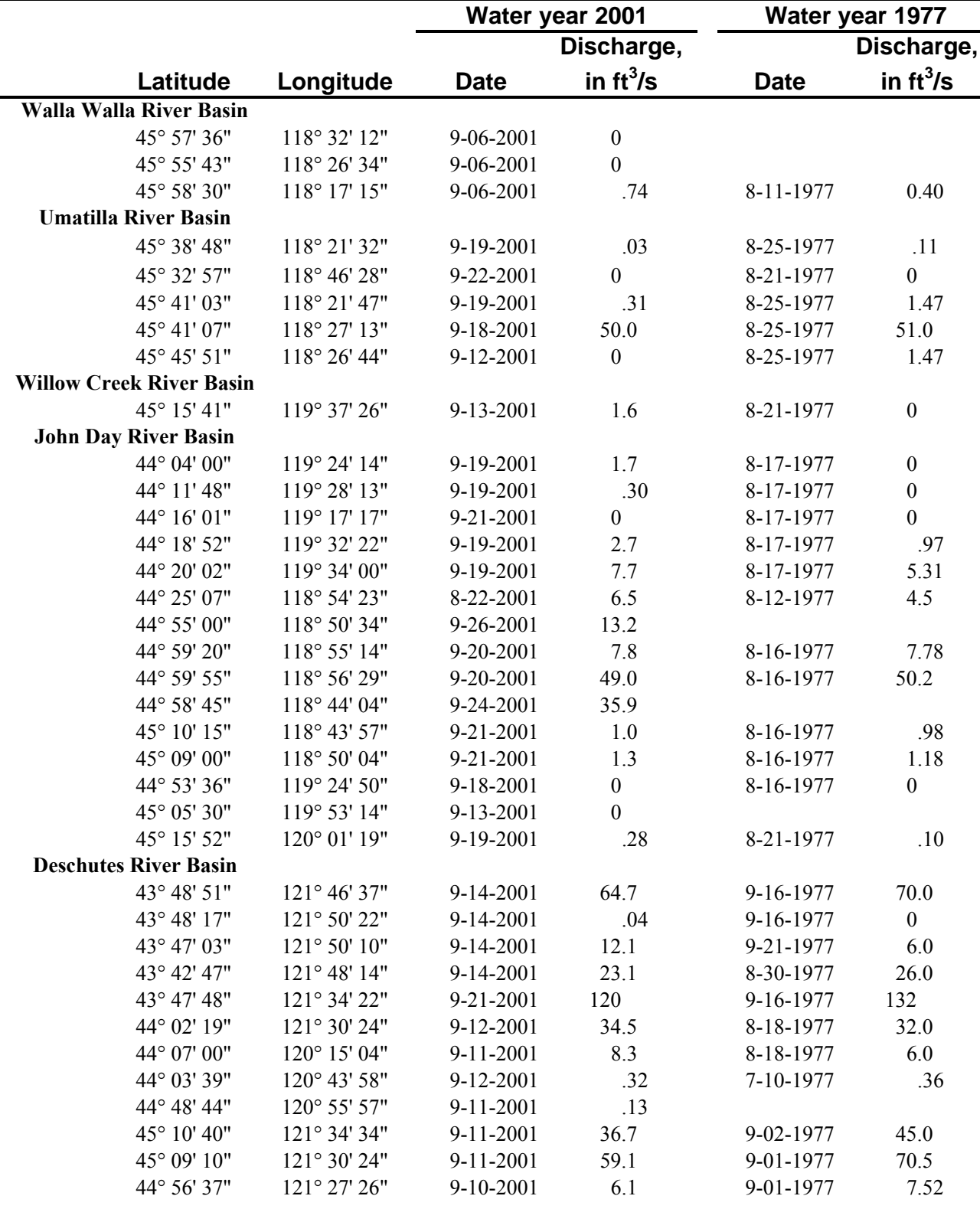


Table 3. Low-flow measurement data for 166 selected sites in Oregon, water years 1977 and 2001--Continued

\begin{tabular}{|c|c|c|c|c|c|c|c|}
\hline \multirow{2}{*}{$\begin{array}{l}\text { Gaging- } \\
\text { station } \\
\text { number }\end{array}$} & \multirow[b]{2}{*}{ Gaging-station name } & \multirow[b]{2}{*}{ Latitude } & \multirow[b]{2}{*}{ Longitude } & \multicolumn{2}{|c|}{ Water year 2001} & \multicolumn{2}{|c|}{ Water year 1977} \\
\hline & & & & Date & $\begin{array}{c}\text { Discharge, } \\
\text { in } \mathrm{ft}^{3} / \mathrm{s}\end{array}$ & Date & $\begin{array}{c}\text { Discharge, } \\
\text { in } \mathrm{ft}^{3} / \mathrm{s} \\
\end{array}$ \\
\hline \multicolumn{8}{|c|}{ Mosier Creek Basin } \\
\hline 14113200 & Mosier Creek near Mosier, OR & $\begin{array}{l}45^{\circ} 38^{\prime} 54^{\prime \prime} \\
\text { jer Basin }\end{array}$ & $121^{\circ} 22^{\prime} 39^{\prime \prime}$ & $9-11-2001$ & 1.7 & 8-17-1977 & 0.72 \\
\hline 14113400 & Dog River near Parkdale, OR & $45^{\circ} 24^{\prime} 29^{\prime \prime}$ & $121^{\circ} 31^{\prime} 14^{\prime \prime}$ & $9-11-2001$ & 1.5 & 9-01-1977 & 1.28 \\
\hline 14118500 & West Fork Hood River near Dee, OR & $45^{\circ} 35^{\prime} 54^{\prime \prime}$ & $121^{\circ} 38^{\prime} 09^{\prime \prime}$ & $9-12-2001$ & 97.0 & $8-23-1977$ & 115 \\
\hline \multicolumn{8}{|c|}{ Sandy River Basin } \\
\hline 14131400 & Zigzag River near Rhododendron, OR & $45^{\circ} 18^{\prime} 31^{\prime \prime}$ & $121^{\circ} 51^{\prime} 35^{\prime \prime}$ & $9-10-2001$ & 44.8 & & \\
\hline 14134000 & Salmon River near Government Camp, OR & $45^{\circ} 16^{\prime} 00^{\prime \prime}$ & $121^{\circ} 43^{\prime} 04^{\prime \prime}$ & $9-10-2001$ & .36 & 8-23-1977 & 20.0 \\
\hline \multicolumn{8}{|c|}{ Willamette River Basin } \\
\hline 14144800 & Middle Fork Willamette River near Oakridge, OR & $43^{\circ} 35^{\prime} 49^{\prime \prime}$ & $122^{\circ} 27^{\prime} 24^{\prime \prime}$ & $9-11-2001$ & 193 & $9-15-1977$ & 187 \\
\hline 14146000 & Salt Creek near Oakridge, OR & $43^{\circ} 43^{\prime} 40^{\prime \prime}$ & $122^{\circ} 25^{\prime} 34^{\prime \prime}$ & $9-11-2001$ & 81.2 & 8-10-1977 & 86.6 \\
\hline 14146500 & Salmon Creek near Oakridge, OR & $43^{\circ} 45^{\prime} 44^{\prime \prime}$ & $122^{\circ} 22^{\prime} 22^{\prime \prime}$ & $9-11-2001$ & 98.7 & 8-18-1977 & 122 \\
\hline 14147500 & North Fork of Middle Fork Willamette River near Oakridge, OR & $43^{\circ} 45^{\prime} 29^{\prime \prime}$ & $122^{\circ} 30^{\prime} 34^{\prime \prime}$ & $9-11-2001$ & 111 & 8-19-1977 & 105 \\
\hline 14151500 & Little Fall Creek near Fall Creek, OR & $43^{\circ} 58^{\prime} 09^{\prime \prime}$ & $122^{\circ} 45^{\prime} 24^{\prime \prime}$ & $9-11-2001$ & 9.5 & 8-10-1977 & 13.6 \\
\hline 14152500 & Coast Fork Willamette River at London, OR & $43^{\circ} 38^{\prime} 30^{\prime \prime}$ & $123^{\circ} 05^{\prime} 09^{\prime \prime}$ & $9-11-2001$ & 7.0 & $8-22-1977$ & 10.4 \\
\hline 14156500 & Mosby Creek at mouth near Cottage Grove, OR & $43^{\circ} 46^{\prime} 34^{\prime \prime}$ & $122^{\circ} 59^{\prime} 59^{\prime \prime}$ & $9-11-2001$ & 0 & 8-16-1977 & 6.0 \\
\hline 14159030 & Separation Creek near McKenzie Bridge, OR & $44^{\circ} 07^{\prime} 28^{\prime \prime}$ & $122^{\circ} 02^{\prime} 12^{\prime \prime}$ & $9-10-2001$ & 243 & 8-11-1977 & 236 \\
\hline 14159100 & Horse Creek near McKenzie Bridge, OR & $44^{\circ} 09^{\prime} 47^{\prime \prime}$ & $122^{\circ} 03^{\prime} 36^{\prime \prime}$ & $9-07-2001$ & 234 & 8-11-1977 & 240 \\
\hline 14159190 & French Pete Creek near Rainbow, OR & $44^{\circ} 02^{\prime} 31^{\prime \prime}$ & $122^{\circ} 11^{\prime} 44^{\prime \prime}$ & $9-06-2001$ & 9.1 & 8-09-1977 & 13.5 \\
\hline--- & Quartz Creek near Finn Rock, OR & $44^{\circ} 07^{\prime} 18^{\prime \prime}$ & $122^{\circ} 22^{\prime} 26^{\prime \prime}$ & $9-04-2001$ & 7.1 & 8-09-1977 & 10.1 \\
\hline--- & Lost Creek at Rattlesnake Road near Dexter, OR & $43^{\circ} 54^{\prime} 06^{\prime \prime}$ & $122^{\circ} 49^{\prime} 28^{\prime \prime}$ & $9-12-2001$ & 2.4 & & \\
\hline 14163000 & Gate Creek at Vida, OR & $44^{\circ} 08^{\prime} 44^{\prime \prime}$ & $122^{\circ} 34^{\prime} 19^{\prime \prime}$ & $9-04-2001$ & 14.7 & 8-18-1977 & 17.0 \\
\hline 14167000 & Coyote Creek near Crow, OR & $44^{\circ} 01^{\prime} 18^{\prime \prime}$ & $123^{\circ} 15^{\prime} 21^{\prime \prime}$ & $9-12-2001$ & .04 & 8-26-1977 & 0 \\
\hline 14170500 & Rock Creek near Philomath, OR & $44^{\circ} 30^{\prime} 04^{\prime \prime}$ & $123^{\circ} 26^{\prime} 24^{\prime \prime}$ & $9-13-2001$ & 1.5 & 8-17-1977 & 1.8 \\
\hline 14172000 & Calapooia River at Holley, OR & $44^{\circ} 21^{\prime} 04^{\prime \prime}$ & $122^{\circ} 47^{\prime} 14^{\prime \prime}$ & $9-14-2001$ & 14.7 & 8-19-1977 & 24.0 \\
\hline 14185800 & Middle Santiam River near Cascadia, OR & $44^{\circ} 30^{\prime} 55^{\prime \prime}$ & $122^{\circ} 22^{\prime} 19^{\prime \prime}$ & $9-13-2001$ & 22.8 & $9-16-1977$ & 58.0 \\
\hline--- & Little North Santiam River at Elkhorn, OR & $44^{\circ} 50^{\prime} 09^{\prime \prime}$ & $122^{\circ} 21^{\prime} 20^{\prime \prime}$ & $9-11-2001$ & 13.1 & $7-12-1977$ & 38.8 \\
\hline--- & Marion Forks Creek near Marion Forks, OR & $44^{\circ} 35^{\prime} 59^{\prime \prime}$ & $121^{\circ} 56^{\prime} 16^{\prime \prime}$ & $9-12-2001$ & 92.8 & $9-15-1977$ & 79.1 \\
\hline--- & North Fork Breitenbrush River below Breitenbrush Lake, OR & $44^{\circ} 45^{\prime} 56^{\prime \prime}$ & $121^{\circ} 47^{\prime} 01^{\prime \prime}$ & $9-11-2001$ & .02 & & \\
\hline 14188800 & Thomas Creek near Scio, OR & $44^{\circ} 42^{\prime} 42^{\prime \prime}$ & $122^{\circ} 45^{\prime} 59^{\prime \prime}$ & $9-13-2001$ & 13.7 & 8-18-1977 & 15.0 \\
\hline 14188700 & Crabtree Creek near Crabtree, OR & $44^{\circ} 39^{\prime} 15^{\prime \prime}$ & $122^{\circ} 51^{\prime} 09^{\prime \prime}$ & $9-13-2001$ & 19.2 & & \\
\hline 14190660 & Rickreall Creek above Mercer Reservoir near Falls City, OR & $44^{\circ} 54^{\prime} 04^{\prime \prime}$ & $123^{\circ} 29^{\prime} 37^{\prime \prime}$ & $9-14-2001$ & 2.8 & & \\
\hline 14192500 & South Yamhill River near Willamina, OR & $45^{\circ} 02^{\prime} 49^{\prime \prime}$ & $123^{\circ} 30^{\prime} 14^{\prime \prime}$ & $10-08-2001$ & 12.8 & 8-17-1977 & 7.3 \\
\hline 14193000 & Willamina Creek near Willamina, OR & $45^{\circ} 08^{\prime} 35^{\prime \prime}$ & $123^{\circ} 29^{\prime} 40^{\prime \prime}$ & $10-05-2001$ & 13.1 & 8-17-1977 & 8.3 \\
\hline 14194300 & North Yamhill River near Fairdale, OR & $45^{\circ} 21^{\prime} 54^{\prime \prime}$ & $123^{\circ} 22^{\prime} 44^{\prime \prime}$ & $9-19-2001$ & 2.6 & 8-13-1977 & 2.6 \\
\hline 14198500 & Mollalla River near Wilhoit, OR & $45^{\circ} 00^{\prime} 34^{\prime \prime}$ & $122^{\circ} 28^{\prime} 49^{\prime \prime}$ & $9-06-2001$ & 27.6 & 8-18-1977 & 51.0 \\
\hline 14201500 & Butte Creek at Monitor, OR & $45^{\circ} 06^{\prime} 06^{\prime \prime}$ & $122^{\circ} 44^{\prime} 46^{\prime \prime}$ & $9-13-2001$ & 3.3 & 8-11-1977 & 1.2 \\
\hline 14202510 & Tualatin River near Gaston, OR & $45^{\circ} 26^{\prime} 19^{\prime \prime}$ & $123^{\circ} 08^{\prime} 27^{\prime \prime}$ & $9-08-2001$ & 16.7 & & \\
\hline 14202850 & Scoggins Creek above Hagg Lake near Gaston, OR & $45^{\circ} 30^{\prime} 06^{\prime \prime}$ & $123^{\circ} 15^{\prime} 11^{\prime \prime}$ & $9-25-2001$ & 1.8 & & \\
\hline 14204500 & Gales Creek near Forest Grove, OR & $45^{\circ} 33^{\prime} 20^{\prime \prime}$ & $123^{\circ} 11^{\prime} 09^{\prime \prime}$ & $9-14-2001$ & 6.9 & 8-13-1977 & 3.5 \\
\hline 14205780 & East Fork Dairy Creek near Roy, OR & $45^{\circ} 34^{\prime} 43^{\prime \prime}$ & $123^{\circ} 04^{\prime} 15^{\prime \prime}$ & $9-14-2001$ & 5.1 & 9-16-1977 & 6.0 \\
\hline
\end{tabular}


Table 3. Low-flow measurement data for 166 selected sites in Oregon, water years 1977 and 2001--Continued

\begin{tabular}{|c|c|c|c|c|c|c|c|}
\hline \multirow{2}{*}{$\begin{array}{l}\text { Gaging- } \\
\text { station } \\
\text { number }\end{array}$} & \multirow[b]{2}{*}{ Gaging-station name } & \multirow[b]{2}{*}{ Latitude } & \multirow[b]{2}{*}{ Longitude } & \multicolumn{2}{|c|}{ Water year 2001} & \multicolumn{2}{|c|}{ Water year 1977} \\
\hline & & & & Date & $\begin{array}{c}\text { Discharge, } \\
\text { in } \mathrm{ft}^{3} / \mathrm{s}\end{array}$ & Date & $\begin{array}{c}\text { Discharge, } \\
\text { in } \mathrm{ft}^{3} / \mathrm{s}\end{array}$ \\
\hline \multicolumn{8}{|c|}{ Willamette River Basin--Continued } \\
\hline 14206450 & Rock Creek near Hillsboro, OR & $45^{\circ} 30^{\prime} 08^{\prime \prime}$ & $122^{\circ} 56^{\prime} 52^{\prime \prime}$ & $8-20-2001$ & 5.8 & $9-15-1977$ & 13.7 \\
\hline 14208000 & Clackamas River at Big Bottom, OR & $45^{\circ} 01^{\prime} 00^{\prime \prime}$ & $121^{\circ} 55^{\prime} 14^{\prime \prime}$ & $9-11-2001$ & 207 & & \\
\hline--- & Eagle Creek at Bonny Lure State Park, OR & $45^{\circ} 21^{\prime} 06^{\prime \prime}$ & $122^{\circ} 23^{\prime} 01^{\prime \prime}$ & $9-11-2001$ & 23.1 & & \\
\hline 14211000 & Clackamas River near Clackamas, OR & $45^{\circ} 23^{\prime} 35^{\prime \prime}$ & $122^{\circ} 31^{\prime} 58^{\prime \prime}$ & $9-11-2001$ & 744 & 8-18-1977 & 580 \\
\hline 14211542 & Crystal Springs Creek at Bybee St., OR & $45^{\circ} 28^{\prime} 27^{\prime \prime}$ & $122^{\circ} 38^{\prime} 31^{\prime \prime}$ & $9-12-2001$ & 11.0 & $9-26-1977$ & 12.5 \\
\hline \multicolumn{8}{|c|}{ Columbia River Main Stem } \\
\hline 14247000 & Clatskanie River near Clatskanie, OR & $46^{\circ} 02^{\prime} 54^{\prime \prime}$ & $123^{\circ} 07^{\prime} 24^{\prime \prime}$ & $9-11-2001$ & 8.1 & & \\
\hline 14251500 & Young's River near Astoria, OR & $46^{\circ} 04^{\prime} 00^{\prime \prime}$ & $123^{\circ} 47^{\prime} 25^{\prime \prime}$ & $9-11-2001$ & 12.4 & & \\
\hline \multicolumn{8}{|c|}{ Nehalem River Basin } \\
\hline 14299800 & Nehalem River near Vernonia, OR & $45^{\circ} 47^{\prime} 59^{\prime \prime}$ & $123^{\circ} 17^{\prime} 35^{\prime \prime}$ & $9-11-2001$ & 4.4 & & \\
\hline --- & Salmonberry River near Salmonberry, OR & $45^{\circ} 45^{\prime} 02^{\prime \prime}$ & $123^{\circ} 39^{\prime} 07^{\prime \prime}$ & $9-11-2001$ & 33.4 & & \\
\hline \multicolumn{8}{|c|}{ Trask River Basin } \\
\hline \multicolumn{8}{|c|}{ Salmon River Basin } \\
\hline 14303748 & \multicolumn{6}{|c|}{ Siletz River Basin } & 33.2 \\
\hline \multicolumn{8}{|c|}{ Yaquina River Basin } \\
\hline 14306010 & Rocky Creek near Depoe Bay, OR & $44^{\circ} 44^{\prime} 12^{\prime \prime}$ & $124^{\circ} 01^{\prime} 46^{\prime \prime}$ & $9-12-2001$ & 1.8 & 8-16-1977 & 1.8 \\
\hline--- & Big Elk Creek near Elk City, OR & $44^{\circ} 36^{\prime} 24^{\prime \prime}$ & $123^{\circ} 51^{\prime} 12^{\prime \prime}$ & $9-12-2001$ & 9.8 & 8-17-1977 & 8.4 \\
\hline 14306030 & Yaquina River near Chitwood, OR & $44^{\circ} 39^{\prime} 29^{\prime \prime}$ & $123^{\circ} 50^{\prime} 19^{\prime \prime}$ & $9-15-2001$ & 4.2 & 8-18-1977 & 7.3 \\
\hline \multicolumn{8}{|c|}{ Alsea River Basin } \\
\hline 14306400 & Five Rivers near Fisher, OR & $44^{\circ} 20^{\prime} 14^{\prime \prime}$ & $123^{\circ} 49^{\prime} 39^{\prime \prime}$ & $9-11-2001$ & 23.0 & $8-17-1977$ & 23.0 \\
\hline 14306600 & Drift Creek near Salado, OR & $44^{\circ} 30^{\prime} 49^{\prime \prime}$ & $123^{\circ} 50^{\prime} 54^{\prime \prime}$ & $9-12-2001$ & 7.0 & 8-16-1977 & 6.36 \\
\hline \multicolumn{8}{|c|}{ Tenmile Creek Basin } \\
\hline \multicolumn{8}{|c|}{ Big Creek Basin } \\
\hline 14306900 & \multicolumn{6}{|c|}{ Siuslaw River Basin } & 6.0 \\
\hline 14306920 & Siuslaw River near Lorane, OR & $43^{\circ} 51^{\prime} 04^{\prime \prime}$ & $123^{\circ} 22^{\prime} 14^{\prime \prime}$ & $9-12-2001$ & 3.7 & 8-09-1977 & 1.51 \\
\hline 14306980 & Wolf Creek near Austa, OR & $43^{\circ} 58^{\prime} 00^{\prime \prime}$ & $123^{\circ} 36^{\prime} 34^{\prime \prime}$ & $9-13-2001$ & 5.7 & 8-10-1977 & 5.88 \\
\hline 14307000 & Siuslaw River above Wildcat at Austa, OR & $43^{\circ} 59^{\prime} 35^{\prime \prime}$ & $123^{\circ} 37^{\prime} 24^{\prime \prime}$ & $9-13-2001$ & 17.3 & $8-10-1977$ & 18.7 \\
\hline 14307500 & Lake Creek at Triangle Lake, OR & $44^{\circ} 09^{\prime} 39^{\prime \prime}$ & $123^{\circ} 34^{\prime} 14^{\prime \prime}$ & $9-13-2001$ & 5.8 & $8-11-1977$ & 2.93 \\
\hline 14307600 & Indian Creek near Rainrock, OR & $44^{\circ} 06^{\prime} 44^{\prime \prime}$ & $123^{\circ} 50^{\prime} 59^{\prime \prime}$ & $9-13-2001$ & 14.1 & $8-11-1977$ & 13.9 \\
\hline 14307625 & Knowles Creek near Mapleton, OR & $44^{\circ} 01^{\prime} 40^{\prime \prime}$ & $123^{\circ} 50^{\prime} 14^{\prime \prime}$ & $9-12-2001$ & .32 & $8-11-1977$ & .24 \\
\hline 14307645 & North Fork Siuslaw River near Minerva, OR & $44^{\circ} 02^{\prime} 49^{\prime \prime}$ & $124^{\circ} 00^{\prime} 14^{\prime \prime}$ & $9-13-2001$ & 16.2 & $8-17-1977$ & 12.0 \\
\hline \multicolumn{8}{|c|}{ Umpqua River Basin } \\
\hline --- & Middle Creek at mouth near Riddle, OR & $42^{\circ} 48^{\prime} 42^{\prime \prime}$ & $123^{\circ} 35^{\prime} 49^{\prime \prime}$ & $9-12-2001$ & .56 & 9-16-1977 & 1.41 \\
\hline 14307700 & Jackson Creek near Tiller, OR & $42^{\circ} 57^{\prime} 15^{\prime \prime}$ & $122^{\circ} 49^{\prime} 44^{\prime \prime}$ & $9-12-2001$ & 10.8 & $8-17-1977$ & 14.3 \\
\hline--- & North Umpqua at Highway 60 above Spring River, OR & $43^{\circ} 18^{\prime} 35^{\prime \prime}$ & $122^{\circ} 07^{\prime} 15^{\prime \prime}$ & $9-13-2001$ & 26.8 & & \\
\hline--- & North Umpqua below Spring River, OR & $43^{\circ} 18^{\prime} 38^{\prime \prime}$ & $122^{\circ} 09^{\prime} 22^{\prime \prime}$ & $9-13-2001$ & 259 & & \\
\hline
\end{tabular}


Table 3. Low-flow measurement data for 166 selected sites in Oregon, water years 1977 and 2001--Continued

\begin{tabular}{|c|c|c|c|c|c|c|c|}
\hline \multirow{2}{*}{$\begin{array}{l}\text { Gaging- } \\
\text { station } \\
\text { number }\end{array}$} & \multirow[b]{2}{*}{ Gaging-station name } & \multirow[b]{2}{*}{ Latitude } & \multirow[b]{2}{*}{ Longitude } & \multicolumn{2}{|c|}{ Water year 2001} & \multicolumn{2}{|c|}{ Water year 1977} \\
\hline & & & & Date & $\begin{array}{c}\text { Discharge, } \\
\text { in } \mathrm{ft}^{3} / \mathrm{s}\end{array}$ & Date & $\begin{array}{c}\text { Discharge, } \\
\text { in } \mathrm{ft}^{3} / \mathrm{s} \\
\end{array}$ \\
\hline \multicolumn{8}{|c|}{ Umpqua River Basin--Continued } \\
\hline 14308500 & Elk Creek near Drew, OR & $42^{\circ} 53^{\prime} 25^{\prime \prime}$ & $122^{\circ} 55^{\prime} 04^{\prime \prime}$ & $9-12-2001$ & 0 & 8-18-1977 & 0 \\
\hline 14308700 & Days Creek at Days Creek, OR & $42^{\circ} 58^{\prime} 54^{\prime \prime}$ & $123^{\circ} 08^{\prime} 59^{\prime \prime}$ & $9-17-2001$ & .14 & & \\
\hline 14311000 & North Myrtle Creek near Myrtle Creek, OR & $43^{\circ} 02^{\prime} 30^{\prime \prime}$ & $123^{\circ} 15^{\prime} 34^{\prime \prime}$ & $9-12-2001$ & 1.3 & $8-31-1977$ & 2.27 \\
\hline 14312400 & Silent Creek near Diamond Lake, OR & $43^{\circ} 07^{\prime} 36^{\prime \prime}$ & $122^{\circ} 09^{\prime} 37^{\prime \prime}$ & $9-13-2001$ & 25.1 & $8-07-1977$ & 24.0 \\
\hline 14317600 & Rock Creek near Glide, OR & $43^{\circ} 20^{\prime} 44^{\prime \prime}$ & $122^{\circ} 59^{\prime} 34^{\prime \prime}$ & $9-19-2001$ & 11.2 & $8-10-1977$ & 23.1 \\
\hline 14317800 & Cavitt Creek near Peel, OR & $43^{\circ} 13^{\prime} 19^{\prime \prime}$ & $123^{\circ} 01^{\prime} 09^{\prime \prime}$ & $9-25-2001$ & 5.3 & & \\
\hline 14321400 & Elk Creek near Elkhead, OR & $43^{\circ} 35^{\prime} 44^{\prime \prime}$ & $123^{\circ} 11^{\prime} 39^{\prime \prime}$ & 9-13-2001 & .88 & & \\
\hline \multicolumn{8}{|c|}{ Smith River Basin } \\
\hline 14323100 & Smith River near Gardiner, OR & $\begin{array}{l}43^{\circ} 47^{\prime} 04^{\prime \prime} \\
\text { ver Basin }\end{array}$ & $123^{\circ} 48^{\prime} 54^{\prime \prime}$ & $9-11-2001$ & .60 & & \\
\hline 14323200 & Ten Mile Creek near Lakeside, OR & $43^{\circ} 34^{\prime} 34^{\prime \prime}$ & $124^{\circ} 11^{\prime} 35^{\prime \prime}$ & $9-10-2001$ & 16.0 & & \\
\hline 14323300 & Eel Creek at Lakeside, OR & $43^{\circ} 34^{\prime} 39^{\prime \prime}$ & $124^{\circ} 11^{\prime} 22^{\prime \prime}$ & $9-10-2001$ & 2.1 & $8-17-1977$ & 2.29 \\
\hline 14323500 & Tioga Creek near Tioga, OR & $43^{\circ} 15^{\prime} 54^{\prime \prime}$ & $123^{\circ} 48^{\prime} 44^{\prime \prime}$ & $9-11-2001$ & .86 & $8-17-1977$ & 1.51 \\
\hline 14324500 & West Fork Millicoma River near Allegany, OR & $43^{\circ} 28^{\prime} 34^{\prime \prime}$ & $124^{\circ} 03^{\prime} 25^{\prime \prime}$ & $9-12-2001$ & 4.5 & $8-14-1977$ & 5.6 \\
\hline \multicolumn{8}{|c|}{ Coquille River Basin } \\
\hline--- & East Fork Coquille River near Dora, OR & $43^{\circ} 09^{\prime} 24^{\prime \prime}$ & $123^{\circ} 59^{\prime} 20^{\prime \prime}$ & $9-13-2001$ & 10.2 & 8-17-1977 & 4.02 \\
\hline--- & Elk Creek near Gravelford, OR & $43^{\circ} 06^{\prime} 38^{\prime \prime}$ & $124^{\circ} 01^{\prime} 04^{\prime \prime}$ & 9-13-2001 & .28 & 8-18-1977 & 48 \\
\hline 14326800 & North Fork Coquille River near Fairview, OR & $43^{\circ} 10^{\prime} 45^{\prime \prime}$ & $124^{\circ} 05^{\prime} 10^{\prime \prime}$ & $9-13-2001$ & 7.2 & $8-17-1977$ & 11.1 \\
\hline \multicolumn{8}{|c|}{ Elk River Basin } \\
\hline 14327150 & Sixes River at Sixes, OR & $42^{\circ} 49^{\prime} 05^{\prime \prime}$ & $124^{\circ} 29^{\prime} 00^{\prime \prime}$ & $9-12-2001$ & 16.6 & & \\
\hline 14327300 & Elk River near Sixes, OR & $42^{\circ} 47^{\prime} 44^{\prime \prime}$ & $124^{\circ} 29^{\prime} 20^{\prime \prime}$ & $9-12-2001$ & 51.3 & & \\
\hline \multicolumn{8}{|c|}{ Rogue River Basin } \\
\hline--- & Whisky Creek near Rim Village, OR & $42^{\circ} 54^{\prime} 46^{\prime \prime}$ & $122^{\circ} 19^{\prime} 11^{\prime \prime}$ & $9-12-2001$ & .38 & $9-20-1977$ & .30 \\
\hline--- & Thousand Springs near Castle Point, OR & $42^{\circ} 52^{\prime} 58^{\prime \prime}$ & $122^{\circ} 17^{\prime} 15^{\prime \prime}$ & 9-13-2001 & 27.6 & 9-20-1977 & 28.0 \\
\hline 14333500 & Red Blanket Creek near Prospect, OR & $42^{\circ} 46^{\prime} 40^{\prime \prime}$ & $122^{\circ} 25^{\prime} 39^{\prime \prime}$ & $9-13-2001$ & 49.6 & 9-15-1977 & 44.0 \\
\hline 14335500 & South Fork Big Butte near Butte Falls, OR & $42^{\circ} 32^{\prime} 25^{\prime \prime}$ & $122^{\circ} 33^{\prime} 19^{\prime \prime}$ & $9-11-2001$ & 67.9 & 9-13-1977 & 52.0 \\
\hline 14353000 & West Fork Ashland Creek near Ashland, OR & $42^{\circ} 08^{\prime} 30^{\prime \prime}$ & $122^{\circ} 43^{\prime} 14^{\prime \prime}$ & $9-11-2001$ & 2.1 & 9-06-1977 & 1.4 \\
\hline 14353500 & East Fork Ashland Creek near Ashland, OR & $42^{\circ} 09^{\prime} 10^{\prime \prime}$ & $122^{\circ} 42^{\prime} 34^{\prime \prime}$ & $9-11-2001$ & 2.1 & 9-06-1977 & 1.6 \\
\hline--- & Galls Creek near Rock Point, OR & $42^{\circ} 25^{\prime} 59^{\prime \prime}$ & $123^{\circ} 04^{\prime} 15^{\prime \prime}$ & $9-11-2001$ & 0 & 8-08-1977 & .05 \\
\hline 14361590 & Middle Fork Applegate River near Copper, OR & $42^{\circ} 00^{\prime} 22^{\prime \prime}$ & $123^{\circ} 09^{\prime} 27^{\prime \prime}$ & $9-12-2001$ & 6.2 & & \\
\hline 14361600 & Elliott Creek near Copper, OR & $42^{\circ} 00^{\prime} 15^{\prime \prime}$ & $123^{\circ} 09^{\prime} 04^{\prime \prime}$ & $9-12-2001$ & 6.6 & $9-07-1977$ & 6.9 \\
\hline 14361700 & Carberry Creek near Copper, OR & $42^{\circ} 01^{\prime} 33^{\prime \prime}$ & $123^{\circ} 10^{\prime} 14^{\prime \prime}$ & $9-12-2001$ & 5.9 & 9-16-1977 & 4.2 \\
\hline 14371500 & Grave Creek at Pease Bridge near Placer, OR & $42^{\circ} 38^{\prime} 30^{\prime \prime}$ & $123^{\circ} 12^{\prime} 44^{\prime \prime}$ & $9-11-2001$ & 0 & 8-22-1977 & .37 \\
\hline 14372500 & East Fork Illinois River near Takilma, OR & $42^{\circ} 04^{\prime} 09^{\prime \prime}$ & $123^{\circ} 37^{\prime} 34^{\prime \prime}$ & $9-10-2001$ & 5.4 & 9-14-1977 & 5.4 \\
\hline 14373500 & Althouse Creek near Holland, OR & $42^{\circ} 05^{\prime} 59^{\prime \prime}$ & $123^{\circ} 31^{\prime} 34^{\prime \prime}$ & $9-12-2001$ & 5.7 & 9-05-1977 & 4.28 \\
\hline 14375100 & Sucker Creek below Little Grayback, OR & $42^{\circ} 09^{\prime} 34^{\prime \prime}$ & $123^{\circ} 28^{\prime} 44^{\prime \prime}$ & $9-10-2001$ & 14.9 & $9-11-1977$ & 15.0 \\
\hline 14375500 & West Fork Illinois River near O'Brien, OR & $42^{\circ} 02^{\prime} 19^{\prime \prime}$ & $123^{\circ} 44^{\prime} 54^{\prime \prime}$ & $9-12-2001$ & 1.7 & $9-12-1977$ & 2.3 \\
\hline 14377500 & Deer Creek near Dryden, OR & $42^{\circ} 15^{\prime} 49^{\prime \prime}$ & $123^{\circ} 27^{\prime} 04^{\prime \prime}$ & $9-12-2001$ & 0 & $9-14-1977$ & .62 \\
\hline 14378200 & Illinois River near Agness, OR & $42^{\circ} 31^{\prime} 14^{\prime \prime}$ & $124^{\circ} 02^{\prime} 39^{\prime \prime}$ & $9-11-2001$ & 145 & $8-17-1977$ & 105 \\
\hline \multicolumn{8}{|c|}{ Chetco River Basin } \\
\hline 14400060 & North Fork Chetco River near Brookings, OR & $42^{\circ} 04^{\prime} 36^{\prime \prime}$ & $124^{\circ} 12^{\prime} 45^{\prime \prime}$ & $9-10-2001$ & 4.0 & 9-15-1977 & 1.45 \\
\hline
\end{tabular}


Table 4 
Table 4. Low-flow measurement data for 155 selected sites in Idaho, Wyoming, Utah, and Nevada, water years 1977 and 2001 [latitude and longitude are based on the North American Datum of 1983 and shown in degrees, minutes, seconds; 3 it, cubic feet per second; ID, Idaho; WY, Wyoming; UT, Utah; NV, Nevada; ,--- no number assigned]

\begin{tabular}{|c|c|c|c|c|c|c|c|}
\hline \multirow{2}{*}{$\begin{array}{l}\text { Gaging- } \\
\text { station } \\
\text { number }\end{array}$} & \multirow[b]{2}{*}{ Gaging-station name } & \multirow[b]{2}{*}{ Latitude } & \multirow[b]{2}{*}{ Longitude } & \multicolumn{2}{|c|}{ Water year 2001} & \multicolumn{2}{|c|}{ Water year 1977} \\
\hline & & & & Date & $\begin{array}{c}\text { Discharge, } \\
\text { in } \mathrm{ft}^{3} / \mathrm{s}\end{array}$ & Date & $\begin{array}{c}\text { Discharge, } \\
\text { in } \mathrm{ft}^{3} / \mathrm{s}\end{array}$ \\
\hline \multicolumn{8}{|c|}{ Bear River Basin } \\
\hline 10125500 & Malad River at Woodruff, ID & $42^{\circ} 01^{\prime} 47^{\prime \prime}$ & $112^{\circ} 13^{\prime} 47^{\prime \prime}$ & 9-19-2001 & 9.0 & & \\
\hline \multicolumn{8}{|c|}{ Kootenai River Basin } \\
\hline 12306800 & Round Prairie Creek near Eastport, ID & $48^{\circ} 57^{\prime} 57^{\prime \prime}$ & $116^{\circ} 12^{\prime} 15^{\prime \prime}$ & $8-29-2001$ & .37 & & \\
\hline \multicolumn{8}{|c|}{ Pend Oreille River Basin } \\
\hline 12392100 & Trapper Creek near Clark Fork, ID & $48^{\circ} 15^{\prime} 54^{\prime \prime}$ & $116^{\circ} 07^{\prime} 07^{\prime \prime}$ & $9-05-2001$ & 0 & & \\
\hline 12392350 & Grouse Creek near Colburn, ID & $48^{\circ} 24^{\prime} 26^{\prime \prime}$ & $116^{\circ} 26^{\prime} 15^{\prime \prime}$ & $9-05-2001$ & 5.1 & & \\
\hline 12392450 & Rapid Lightning Creek near Colburn, ID & $48^{\circ} 22^{\prime} 02^{\prime \prime}$ & $116^{\circ} 24^{\prime} 06^{\prime \prime}$ & 9-05-2001 & 8.0 & & \\
\hline 12392950 & Indian Creek near Coolin, ID & $48^{\circ} 37^{\prime} 37^{\prime \prime}$ & $116^{\circ} 49^{\prime} 14^{\prime \prime}$ & $8-27-2001$ & 6.8 & & \\
\hline 12393600 & Binarch Creek near Coolin, ID & $48^{\circ} 28^{\prime} 10^{\prime \prime}$ & $116^{\circ} 55^{\prime} 20^{\prime \prime}$ & $8-27-2001$ & 2.6 & & \\
\hline \multicolumn{8}{|c|}{ Spokane River Basin } \\
\hline 12412600 & Little North Fork Coeur d'Alene River near Enaville, ID & $47^{\circ} 36^{\prime} 38^{\prime \prime}$ & $116^{\circ} 14^{\prime} 23^{\prime \prime}$ & 9-10-2001 & 18.0 & & \\
\hline 12413100 & Boulder Creek at Mullan, ID & $47^{\circ} 28^{\prime} 11^{\prime \prime}$ & $115^{\circ} 47^{\prime} 45^{\prime \prime}$ & $9-12-2001$ & .83 & & \\
\hline 12413200 & Montgomery Creek near Kellogg, ID & $47^{\circ} 33^{\prime} 19^{\prime \prime}$ & $116^{\circ} 04^{\prime} 22^{\prime \prime}$ & $9-10-2001$ & .10 & & \\
\hline 12413700 & Latour Creek above Baldy Creek near Cataldo, ID & $47^{\circ} 28^{\prime} 10^{\prime \prime}$ & $116^{\circ} 26^{\prime} 20^{\prime \prime}$ & $9-17-2001$ & 3.3 & & \\
\hline 12413800 & Fourth of July Creek above Bently Creek near Cataldo, ID & $47^{\circ} 34^{\prime} 25^{\prime \prime}$ & $116^{\circ} 26^{\prime} 32^{\prime \prime}$ & $9-17-2001$ & .52 & & \\
\hline 12414200 & Marble Creek near Calder, ID & $47^{\circ} 13^{\prime} 38^{\prime \prime}$ & $116^{\circ} 01^{\prime} 12^{\prime \prime}$ & $9-18-2001$ & 45.0 & & \\
\hline 12414750 & Falls Creek near St. Joe, ID & $47^{\circ} 49^{\prime} 15^{\prime \prime}$ & $116^{\circ} 17^{\prime} 51^{\prime \prime}$ & $9-18-2001$ & 2.7 & & \\
\hline 12414800 & Bond Creek at St. Joe, ID & $47^{\circ} 18^{\prime} 25^{\prime \prime}$ & $116^{\circ} 20^{\prime} 34^{\prime \prime}$ & $9-18-2001$ & 2.4 & & \\
\hline 12415000 & Cherry Creek near St. Maries, ID & $47^{\circ} 19^{\prime} 00^{\prime \prime}$ & $116^{\circ} 36^{\prime} 47^{\prime \prime}$ & $9-18-2001$ & .08 & & \\
\hline 12415200 & Plummer Creek tributary at Plummer, ID & $47^{\circ} 20^{\prime} 19^{\prime \prime}$ & $116^{\circ} 53^{\prime} 17^{\prime \prime}$ & $8-29-2001$ & 0 & & \\
\hline 12415300 & Mica Creek near Coeur d'Alene, ID & $47^{\circ} 36^{\prime} 00^{\prime \prime}$ & $116^{\circ} 52^{\prime} 53^{\prime \prime}$ & $8-29-2001$ & 1.2 & & \\
\hline 12422950 & Hangman Creek near Tensed, ID & $47^{\circ} 11^{\prime} 24^{\prime \prime}$ & $117^{\circ} 01^{\prime} 06^{\prime \prime}$ & $8-29-2001$ & .25 & & \\
\hline \multicolumn{8}{|c|}{ Snake River Basin } \\
\hline 13009500 & Lewis River near Moose Falls, ID & $44^{\circ} 09^{\prime} 00^{\prime \prime}$ & $110^{\circ} 40^{\prime} 00^{\prime \prime}$ & 9-29-2001 & 95.0 & & \\
\hline \multicolumn{8}{|c|}{ Hoback River Basin } \\
\hline 13019430 & Granite Creek near Bondurant, WY & $43^{\circ} 17^{\prime} 47^{\prime \prime}$ & $110^{\circ} 30^{\prime} 08^{\prime \prime}$ & $9-26-2001$ & 50.8 & & \\
\hline 13019438 & Little Granite Creek at mouth near Bondurant, WY & $43^{\circ} 17^{\prime} 55^{\prime \prime}$ & $110^{\circ} 31^{\prime} 05^{\prime \prime}$ & $9-26-2001$ & 5.3 & & \\
\hline \multicolumn{8}{|c|}{ Salt River Basin } \\
\hline 13027200 & Bear Canyon Creek near Freedom, WY & $42^{\circ} 58^{\prime} 36^{\prime \prime}$ & $111^{\circ} 11^{\prime} 47^{\prime \prime}$ & 9-13-2001 & .60 & 9-20-1977 & 0.30 \\
\hline \multicolumn{8}{|c|}{ McCoy Creek Basin } \\
\hline 13029500 & McCoy Creek above Reservoir near Alpine, WY & $\quad 43^{\circ} 10^{\prime} 53^{\prime \prime}$ & $111^{\circ} 07^{\prime} 07^{\prime \prime}$ & $9-13-2001$ & 12.5 & 9-02-1977 & 10.6 \\
\hline \multicolumn{7}{|c|}{ Indian Creek Basin } & \\
\hline \multicolumn{8}{|c|}{ Elk Creek Basin } \\
\hline 13030500 & (Big) Elk Creek above Reservoir near Irwin, ID & $43^{\circ} 19^{\prime} 24^{\prime \prime}$ & $111^{\circ} 06^{\prime} 44^{\prime \prime}$ & $9-13-2001$ & 22.1 & 9-02-1977 & 21.3 \\
\hline \multicolumn{8}{|c|}{ Bear Creek Basin } \\
\hline 13032000 & Bear Creek above Reservoir near Irwin, ID & $43^{\circ} 17^{\prime} 00^{\prime \prime}$ & $111^{\circ} 13^{\prime} 17^{\prime \prime}$ & $9-13-2001$ & 18.3 & 9-02-1977 & 15.4 \\
\hline
\end{tabular}


Table 4. Low-flow measurement data for 155 selected sites in Idaho, Wyoming. Utah, and Nevada, water years 1977 and 2001--Continued

\begin{tabular}{|c|c|}
\hline $\begin{array}{l}\text { Gaging- } \\
\text { station } \\
\text { number }\end{array}$ & Gaging-station name \\
\hline 13034000 & Fall Creek near Swan Valley, ID \\
\hline 13037600 & Birch Creek near Heise, ID \\
\hline 13038410 & Lyons Creek near Ririe, ID \\
\hline 13038900 & Targhee Creek near Macks Inn, ID \\
\hline 13040000 & Henrys Fork near Big Springs, ID \\
\hline 13040500 & Big Springs Creek at Big Springs, ID \\
\hline 13040800 & Moose Creek near Big Springs, ID \\
\hline 13043000 & Buffalo River at Island Park, ID \\
\hline 13043820 & Henrys Fork at Pinehaven subdivision near Last Chance, ID \\
\hline 13044000 & Henrys Fork at Warm River, ID \\
\hline 13044500 & Warm River at Warm River, ID \\
\hline 13045500 & Robinson Creek at Warm River, ID \\
\hline 13050598 & Warm Creek near Victor, ID \\
\hline 13050800 & Moose Creek near Victor, ID \\
\hline 13051240 & Fox Creek Springs near Victor, ID \\
\hline 13051270 & Fish Creek near Driggs, ID \\
\hline 13051635 & Six Springs near Driggs, ID \\
\hline 13051710 & Woods Creek near Driggs, ID \\
\hline 13055319 & Moody Creek near Rexburg, ID \\
\hline 13057600 & Homer Creek near Herman, ID \\
\hline 13062683 & Sheep Creek at USFS boundary near Wayan, ID \\
\hline 13062700 & Angus Creek at Road 095 crossing near Henry, ID \\
\hline 13062905 & Slug Creek at Sweet Ranch near Soda Springs, ID \\
\hline 13062960 & Trail Creek at mouth near Soda Springs, ID \\
\hline 13065940 & Wolverine Creek near Goshen, ID \\
\hline 13075300 & East Fork Mink Creek near Pocatello, ID \\
\hline 13076100 & Rattlesnake Creek near Pocatello, ID \\
\hline 13072100 & Portneuf River tributary at Bancroft, ID \\
\hline 13072500 & Pebble Creek near Pebble, ID \\
\hline 13072550 & Portneuf River at Symmon's Road near Lava Hot Springs, ID \\
\hline 13072600 & Portneuf River above Fish Creek near Lava Hot Springs, ID \\
\hline 13072790 & Fish Creek at mouth near Lava Hot Springs, ID \\
\hline 13072810 & Portneuf River below Lava Hot Springs, ID \\
\hline 13073700 & Robbers Roost Creek near McCammon, ID \\
\hline
\end{tabular}

Water year

Latitude

Fall Creek Basin

Birch Creek Basin

$43^{\circ} 36^{\prime} 00^{\prime \prime}$

Lyons Creek Basin

$43^{\circ} 40^{\prime} 54^{\prime \prime}$

Henrys Fork Basin

$44^{\circ} 38^{\prime} 50^{\prime \prime}$

$44^{\circ} 30^{\prime} 40^{\prime \prime}$

$44^{\circ} 29^{\prime} 58^{\prime \prime}$

$44^{\circ} 29^{\prime} 05^{\prime \prime}$

$44^{\circ} 25^{\prime} 19^{\prime \prime}$

$44^{\circ} 17^{\prime} 32^{\prime \prime}$

$44^{\circ} 06^{\prime} 51^{\prime \prime}$

$44^{\circ} 06^{\prime} 56^{\prime \prime}$

$44^{\circ} 06^{\prime} 52^{\prime \prime}$

$43^{\circ} 34^{\prime} 40^{\prime \prime}$

$43^{\circ} 33^{\prime} 48^{\prime \prime}$

$43^{\circ} 38^{\prime} 07^{\prime \prime}$

$43^{\circ} 40^{\prime} 13^{\prime \prime}$

$43^{\circ} 41^{\prime} 51^{\prime \prime}$

$43^{\circ} 43^{\prime} 05^{\prime \prime}$

$43^{\circ} 46^{\prime} 48^{\prime \prime}$

Willow Creek Basin

$43^{\circ} 11^{\prime} 42^{\prime \prime}$

Blackfoot River Basin

$42^{\circ} 51^{\prime} 47^{\prime \prime}$

$42^{\circ} 49^{\prime} 43^{\prime \prime}$

$42^{\circ} 42^{\prime} 24^{\prime \prime}$

$42^{\circ} 45^{\prime} 29^{\prime \prime}$

$43^{\circ} 15^{\prime} 02^{\prime \prime}$

$42^{\circ} 44^{\prime} 20^{\prime \prime}$

$42^{\circ} 42^{\prime} 04^{\prime \prime}$

Portneuf River Basin

$42^{\circ} 43^{\prime} 30^{\prime \prime}$

$42^{\circ} 44^{\prime} 10^{\prime \prime}$

$42^{\circ} 40^{\prime} 28^{\prime \prime}$

$42^{\circ} 37^{\prime} 19^{\prime \prime}$

$42^{\circ} 37^{\prime} 14^{\prime \prime}$

$42^{\circ} 37^{\prime} 23^{\prime \prime}$

$42^{\circ} 42^{\prime} 30^{\prime \prime}$

Longitude

$111^{\circ} 22^{\prime} 42^{\prime \prime}$

$111^{\circ} 43^{\prime} 10^{\prime \prime}$

9-13-2001

$111^{\circ} 44^{\prime} 50^{\prime \prime}$

$111^{\circ} 20^{\prime} 30^{\prime \prime}$

$111^{\circ} 17^{\prime} 23^{\prime \prime}$

$111^{\circ} 15^{\prime} 20^{\prime \prime}$

$111^{\circ} 17^{\prime} 09^{\prime \prime}$

$111^{\circ} 22^{\prime} 17^{\prime \prime}$

$111^{\circ} 27^{\prime} 19^{\prime \prime}$

$111^{\circ} 19^{\prime} 59^{\prime \prime}$

$111^{\circ} 19^{\prime} 09^{\prime \prime}$

$111^{\circ} 19^{\prime} 27^{\prime \prime}$

$111^{\circ} 07^{\prime} 17^{\prime \prime}$

$111^{\circ} 04^{\prime} 04^{\prime \prime}$

$111^{\circ} 08^{\prime} 43^{\prime \prime}$

$111^{\circ} 07^{\prime} 56^{\prime \prime}$

$111^{\circ} 06^{\prime} 58^{\prime \prime}$

$111^{\circ} 09^{\prime} 03^{\prime \prime}$

$111^{\circ} 37^{\prime} 21^{\prime \prime}$

9-04-2001

$111^{\circ} 37^{\prime} 56^{\prime \prime}$

$111^{\circ} 20^{\prime} 04^{\prime \prime}$

$111^{\circ} 20^{\prime} 15^{\prime \prime}$

$111^{\circ} 22^{\prime} 04^{\prime \prime}$

$111^{\circ} 26^{\prime} 50^{\prime \prime}$

$112^{\circ} 00^{\prime} 57^{\prime \prime}$

$112^{\circ} 23^{\prime} 30^{\prime \prime}$

$112^{\circ} 33^{\prime} 28^{\prime \prime}$

$111^{\circ} 54^{\prime} 25^{\prime \prime}$

$112^{\circ} 01^{\prime} 16^{\prime \prime}$

$112^{\circ} 01^{\prime} 12^{\prime \prime}$

$111^{\circ} 59^{\prime} 47^{\prime \prime}$

$111^{\circ} 59^{\prime} 27^{\prime \prime}$

$112^{\circ} 02^{\prime} 06^{\prime \prime}$

$112^{\circ} 12^{\prime} 10^{\prime \prime}$ year 2001

Discharge,

in $\mathrm{ft}^{3} / \mathrm{s}$

Water year 1977

14.6
9-04-2001

9-13-2001

9-13-2001

9-13-2001

9-13-2001

9-13-2001

9-14-2001

9-14-2001

9-14-2001

9-14-2001

9-26-2001

9-10-2001

9-26-2001

8-07-2001

8-08-2001

9-26-2001

9-26-2001

9-04-2001

9-18-2001

9-18-2001

9-18-2001

9-18-2001

9-04-2001

9-19-2001

9-20-2001

9-18-2001

9-18-2001

9-17-2001

9-18-2001

9-18-2001

9-18-2001

9-18-2001
.10

9-09-1977

0.80

3.3

40.9

169

21.8
205

205
794

794

206

56.5

27.3

11.3

.72
3.9

3.9

2.2

$$
0
$$

9-27-1977

1.4

0

9-28-1977

0

9-23-1977 
Table 4. Low-flow measurement data for 155 selected sites in Idaho, Wyoming, Utah, and Nevada, water years 1977 and 2001--Continued

\begin{tabular}{|c|c|}
\hline $\begin{array}{l}\text { Gaging- } \\
\text { station } \\
\text { number }\end{array}$ & Gaging-station name \\
\hline 13077400 & Rock Creek above Dry Hollow near Rockland, ID \\
\hline 13077659 & Raft River near Yost, UT \\
\hline 13084400 & Birch Creek above diversions near Oakley, ID \\
\hline 13088510 & Cottonwood Creek near Oakley, ID \\
\hline 13092500 & McMullen Creek near Rock Creek, ID \\
\hline 13104800 & Shoshone Creek at mouth near San Jacinto, NV \\
\hline 13108200 & West Camas Creek near Kilgore, ID \\
\hline 13108900 & Camas Creek at Red Road near Kilgore, ID \\
\hline 13112300 & Beaver Creek at Humphrey, ID \\
\hline 13112900 & Huntley Canyon at Spencer, ID \\
\hline 13117200 & Main Fork near Goldburg, ID \\
\hline 13117300 & Sawmill Creek near Goldburg, ID \\
\hline 13117600 & Dry Creek below Dry Creek Reservoir near Clyde, ID \\
\hline 13118400 & Wet Creek below Coal Creek near Mackay, ID \\
\hline 13119800 & North Fork Big Lost River near Chilly, ID \\
\hline 13120240 & East Fork Big Lost River at Rosenkance Ranch near Chilly, ID \\
\hline 13129800 & Alder Creek below South Fork near Mackay, ID \\
\hline 13131500 & Pass Creek near Leslie, ID \\
\hline 13137000 & Warm Springs Creek (at Guyer Hot Springs) near Ketchum, ID \\
\hline 13138000 & East Fork Big Wood River at Gimlet, ID \\
\hline 13141350 & Soldier Creek near Fairfield, ID \\
\hline 13145700 & Schooler Creek near Gooding, ID \\
\hline 13147300 & Muldoon Creek near Garfield Guard Station, ID \\
\hline 13149000 & Fish Creek above Fish Creek Dam near Carey, ID \\
\hline 13154000 & Clover Creek near Bliss, ID \\
\hline 13155300 & Little Canyon Creek at Stout Crossing near Glenns Ferry, ID \\
\hline
\end{tabular}

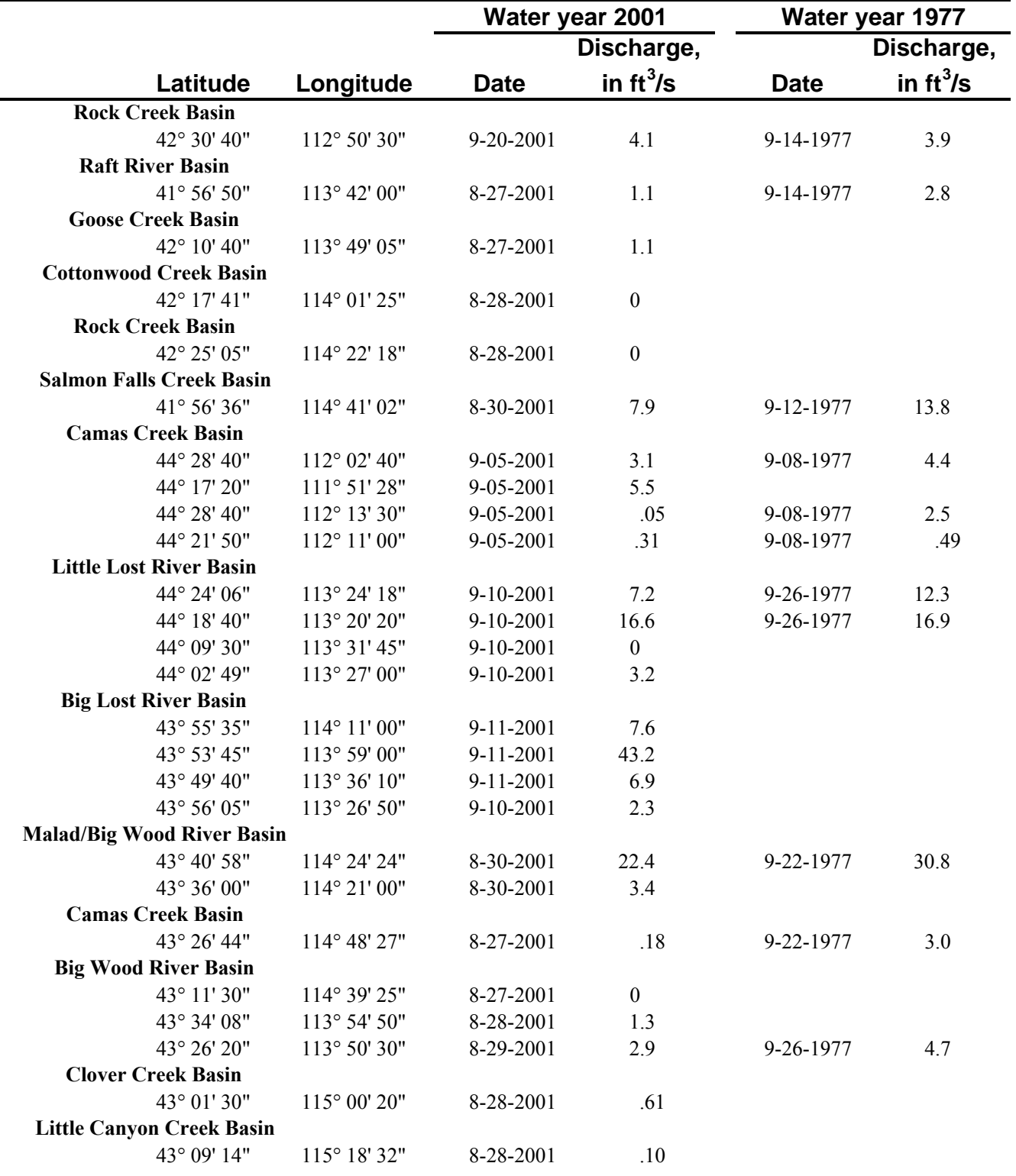


Table 4. Low-flow measurement data for 155 selected sites in Idaho, Wyoming. Utah, and Nevada, water years 1977 and 2001--Continued

\begin{tabular}{cl}
$\begin{array}{c}\text { Gaging- } \\
\text { station }\end{array}$ & \\
number & \\
\hline & \multicolumn{1}{c}{ Gaging-station name } \\
13162300 & Jarbidge River at Buck Creek near Murphy Hot Springs, ID \\
13162500 & East Fork Jarbidge River near Three Creek, ID \\
& \\
13172290 & Sinker Creek above Scotch Bob Creek near Murphy, ID \\
& \\
13177910 & North Fork Owyhee River at Fairylawn, ID \\
& \\
13184200 & Roaring River near Rocky Bar, ID \\
13184800 & Beaver Creek near Lowman, ID \\
13184990 & Alder Creek near Twin Springs, ID \\
13187000 & Fall Creek near Anderson Ranch Dam, ID \\
13193500 & Grouse Creek near Arrowrock Dam, ID \\
13199800 & Grimes Creek at mouth near Idaho City, ID \\
13200500 & Robie Creek near Arrowrock Dam, ID \\
& \\
13234300 & Fivemile Creek near Lowman, ID \\
13234500 & Clear Creek at Lowman, ID \\
13235100 & Rock Creek at Lowman, ID \\
13237300 & Danskin Creek near Grimes Pass, ID \\
13237600 & Cabin Creek near Smiths Ferry, ID \\
13238300 & Deep Creek near McCall, ID \\
13245400 & Tripod Creek at Smiths Ferry, ID \\
13247000 & Porter Creek near Gardena, ID \\
13292200 & Salmon River at head near Obsidian, ID \\
13293800 & Salmon River at Highway 93 above Redfish Creek near Stanley, ID \\
13293900 & Redfish Lake Creek below Redfish Lake near Stanley, ID \\
13296000 & Yankee Fork Salmon River near Clayton, ID \\
13297500 & Big Boulder Creek near Clayton, ID \\
13298000 & East Fork Salmon River near Clayton, ID \\
13301700 & Morse Creek above diversions near May, ID \\
13303000 & Texas Creek near Leadore, ID \\
13305800 & Hughes Creek near North Fork, ID \\
13306000 & North Fork Salmon River at North Fork, ID \\
13306440 & Panther Creek below Big Deer Creek near Salmon, ID \\
13307000 & Salmon River near Shoup, ID \\
13307050 & Owl Creek near Shoup, ID \\
13308500 & Marsh Creek near Cape Horn, ID \\
13309000 & Bear Valley Creek near Cape Horn, ID \\
13310500 & South Fork Salmon River near Knox, ID \\
&
\end{tabular}

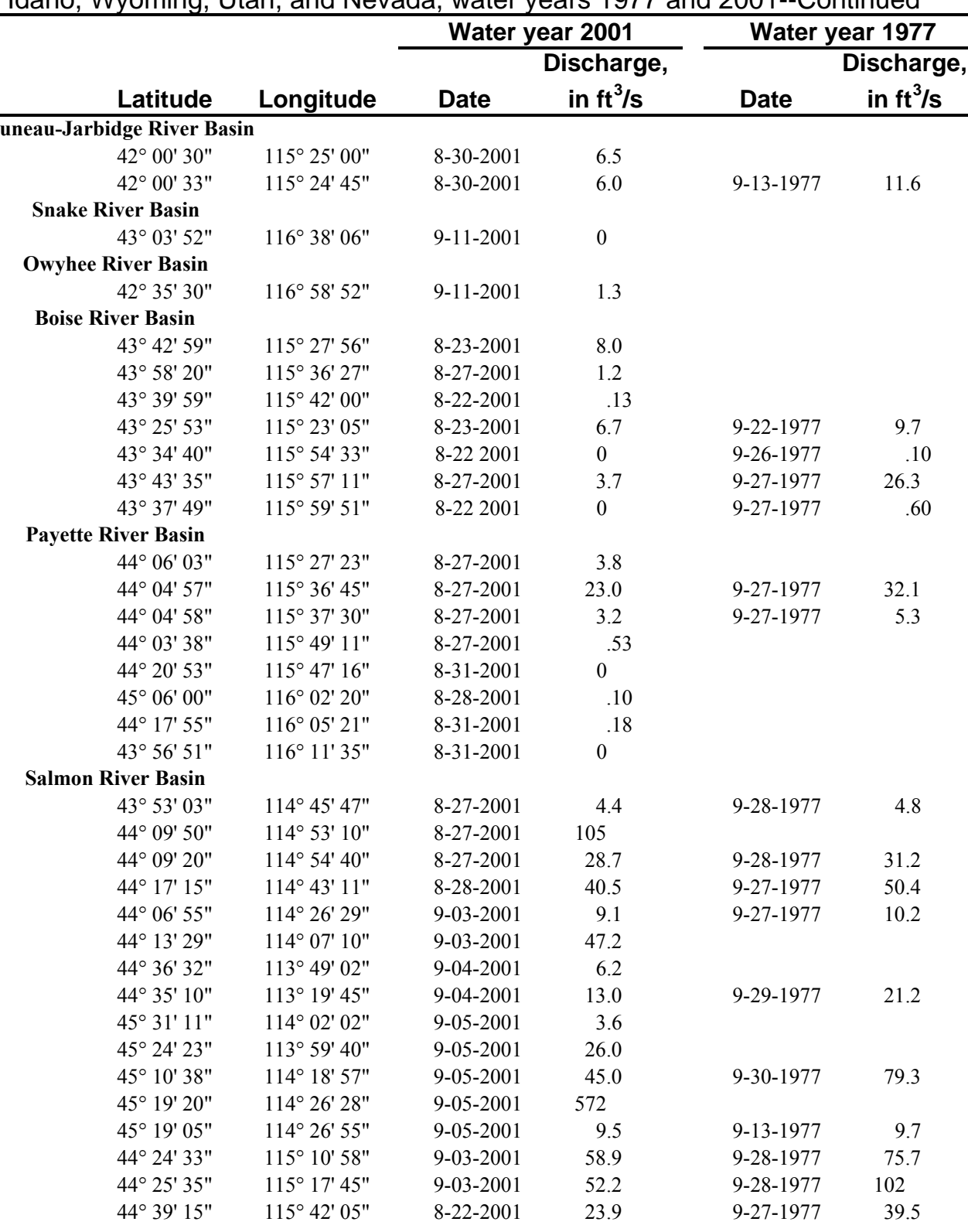


Table 4. Low-flow measurement data for 155 selected sites in Idaho, Wyoming, Utah, and Nevada, water years 1977 and 2001--Continued

\begin{tabular}{|c|c|c|c|c|c|c|c|}
\hline \multirow{2}{*}{$\begin{array}{l}\text { Gaging- } \\
\text { station } \\
\text { number }\end{array}$} & \multirow[b]{2}{*}{ Gaging-station name } & \multirow[b]{2}{*}{ Latitude } & \multirow[b]{2}{*}{ Longitude } & \multicolumn{2}{|c|}{ Water year 2001} & \multicolumn{2}{|c|}{ Water year 1977} \\
\hline & & & & Date & $\begin{array}{c}\text { Discharge, } \\
\text { in } \mathrm{ft}^{3} / \mathrm{s}\end{array}$ & Date & $\begin{array}{c}\text { Discharge, } \\
\text { in } \mathrm{ft}^{3} / \mathrm{s}\end{array}$ \\
\hline \multicolumn{8}{|c|}{ Salmon River Basin--Continued } \\
\hline 13310520 & Dollar Creek near Warm Lake near Cascade, ID & $44^{\circ} 43^{\prime} 13^{\prime \prime}$ & $115^{\circ} 41^{\prime} 41^{\prime \prime}$ & $8-23-2001$ & 5.4 & & \\
\hline 13310565 & Blackmare Creek near Poverty Flat near Cascade, ID & $44^{\circ} 49^{\prime} 20^{\prime \prime}$ & $115^{\circ} 42^{\prime} 18^{\prime \prime}$ & $8-23-2001$ & 6.9 & & \\
\hline 13310570 & South Fork Salmon River at Poverty Flat near Cascade, ID & $44^{\circ} 49^{\prime} 56^{\prime \prime}$ & $115^{\circ} 42^{\prime} 16^{\prime \prime}$ & $8-23-2001$ & 56.6 & & \\
\hline 13310660 & Little Buckhorn Creek near Krassel Ranger Station, ID & $44^{\circ} 54^{\prime} 47^{\prime \prime}$ & $115^{\circ} 44^{\prime} 59^{\prime \prime}$ & $8-23-2001$ & 1.2 & & \\
\hline 13310670 & West Fork Buckhorn Creek near Krassel Ranger Station, ID & $44^{\circ} 55^{\prime} 02^{\prime \prime}$ & $115^{\circ} 44^{\prime} 34^{\prime \prime}$ & $8-23-2001$ & 2.7 & & \\
\hline 13311500 & East Fork of South Fork Salmon River near Stibnite, ID & $44^{\circ} 56^{\prime} 11^{\prime \prime}$ & $115^{\circ} 20^{\prime} 16^{\prime \prime}$ & $8-22-2001$ & 16.0 & 9-28-1977 & 24.2 \\
\hline 13312500 & Johnson Creek near Landmark Ranger Station, ID & $44^{\circ} 40^{\prime} 56^{\prime \prime}$ & $115^{\circ} 32^{\prime} 23^{\prime \prime}$ & $8-22-2001$ & 7.1 & $9-27-1977$ & 20.7 \\
\hline 13313500 & Secesh River near Burgdorf, ID & $45^{\circ} 13^{\prime} 58^{\prime \prime}$ & $115^{\circ} 48^{\prime} 40^{\prime \prime}$ & $8-28-2001$ & 28.3 & $9-29-1977$ & 156 \\
\hline 13314500 & Warren Creek near Warren, ID & $45^{\circ} 16^{\prime} 34^{\prime \prime}$ & $115^{\circ} 41^{\prime} 49^{\prime \prime}$ & $8-28-2001$ & 6.5 & & \\
\hline 13315050 & French Creek at French Creek near Riggins, ID & $45^{\circ} 25^{\prime} 23^{\prime \prime}$ & $116^{\circ} 01^{\prime} 38^{\prime \prime}$ & $8-28-2001$ & 15.8 & $9-21-1977$ & 37.7 \\
\hline 13316000 & Boulder Creek near Tamarack, ID & $45^{\circ} 04^{\prime} 58^{\prime \prime}$ & $116^{\circ} 26^{\prime} 51^{\prime \prime}$ & $8-30-2001$ & .98 & $9-29-1977$ & 2.6 \\
\hline 13316390 & Rapid River above hatchery near Riggins, ID & $45^{\circ} 21^{\prime} 04^{\prime \prime}$ & $116^{\circ} 23^{\prime} 57^{\prime \prime}$ & $9-10-2001$ & 60.1 & & \\
\hline 13316600 & Slate Creek at mouth at Slate Creek, ID & $45^{\circ} 38^{\prime} 24^{\prime \prime}$ & $116^{\circ} 17^{\prime} 01^{\prime \prime}$ & $9-14-2001$ & 28.7 & & \\
\hline 13317045 & White Bird Creek near White Bird, ID & $45^{\circ} 47^{\prime} 19^{\prime \prime}$ & $116^{\circ} 15^{\prime} 29^{\prime \prime}$ & $9-14-2001$ & 5.2 & $9-30-1977$ & 55.2 \\
\hline \multicolumn{8}{|c|}{ Clearwater River Basin } \\
\hline 13336600 & Swiftwater Creek near Lowell, ID & $46^{\circ} 06^{\prime} 55^{\prime \prime}$ & $115^{\circ} 34^{\prime} 23^{\prime \prime}$ & $9-11-2001$ & 2.5 & & \\
\hline 13336650 & East Fork Papoose Creek near Powell Ranger Station, ID & $46^{\circ} 32^{\prime} 07^{\prime \prime}$ & $114^{\circ} 45^{\prime} 55^{\prime \prime}$ & $8-29-2001$ & 1.5 & & \\
\hline 13336800 & Warm Springs Creek near Powell Ranger Station, ID & $46^{\circ} 28^{\prime} 17^{\prime \prime}$ & $114^{\circ} 53^{\prime} 00^{\prime \prime}$ & $8-29-2001$ & 19.4 & & \\
\hline 13336850 & Weir Creek near Powell Ranger Station, ID & $46^{\circ} 27^{\prime} 31^{\prime \prime}$ & $115^{\circ} 02^{\prime} 04^{\prime \prime}$ & $8-29-2001$ & 4.2 & & \\
\hline 13336900 & Fish Creek near Lowell, ID & $46^{\circ} 20^{\prime} 00^{\prime \prime}$ & $115^{\circ} 20^{\prime} 46^{\prime \prime}$ & $8-29-2001$ & 24.9 & & \\
\hline 13337100 & Clear Creek near Kooskia, ID & $46^{\circ} 07^{\prime} 56^{\prime \prime}$ & $115^{\circ} 56^{\prime} 50^{\prime \prime}$ & $8-29-2001$ & 16.7 & & \\
\hline 13337200 & Red Horse Creek near Elk City, ID & $45^{\circ} 47^{\prime} 39^{\prime \prime}$ & $115^{\circ} 24^{\prime} 02^{\prime \prime}$ & $8-30-2001$ & .79 & & \\
\hline 13337520 & Crooked River near mouth near Elk City, ID & $45^{\circ} 49^{\prime} 18^{\prime \prime}$ & $115^{\circ} 31^{\prime} 40^{\prime \prime}$ & $8-30-2001$ & 15.4 & & \\
\hline 13337700 & Peasley Creek near Golden, ID & $45^{\circ} 49^{\prime} 00^{\prime \prime}$ & $115^{\circ} 49^{\prime} 09^{\prime \prime}$ & $8-30-2001$ & 3.0 & & \\
\hline 13338000 & South Fork Clearwater River near Grangeville, ID & $45^{\circ} 54^{\prime} 45^{\prime \prime}$ & $116^{\circ} 00^{\prime} 20^{\prime \prime}$ & $8-30-2001$ & 139 & & \\
\hline 13338200 & Sally Ann Creek near Stites, ID & $46^{\circ} 00^{\prime} 35^{\prime \prime}$ & $115^{\circ} 57^{\prime} 46^{\prime \prime}$ & $8-30-2001$ & .43 & & \\
\hline 13338800 & Lawyer Creek near Nezperce, ID & $46^{\circ} 09^{\prime} 46^{\prime \prime}$ & $116^{\circ} 14^{\prime} 27^{\prime \prime}$ & $8-28-2001$ & 1.7 & $8-24-1977$ & 3.0 \\
\hline 13339700 & Canal Gulch Creek at Pierce Ranger Station, ID & $46^{\circ} 29^{\prime} 49^{\prime \prime}$ & $115^{\circ} 47^{\prime} 45^{\prime \prime}$ & $8-29-2001$ & .18 & & \\
\hline 13339900 & Deer Creek near Orofino, ID & $46^{\circ} 29^{\prime} 30^{\prime \prime}$ & $116^{\circ} 10^{\prime} 39^{\prime \prime}$ & $8-29-2001$ & 0 & & \\
\hline 13341100 & Cold Springs Creek near Craigmont, ID & $46^{\circ} 10^{\prime} 09^{\prime \prime}$ & $116^{\circ} 31^{\prime} 09^{\prime \prime}$ & $8-28-2001$ & 0 & & \\
\hline 13341500 & Potlatch River at Kendrick, ID & $46^{\circ} 36^{\prime} 44^{\prime \prime}$ & $116^{\circ} 39^{\prime} 27^{\prime \prime}$ & $8-28-2001$ & 3.4 & & \\
\hline 13342000 & Mission Creek near Winchester, ID & $46^{\circ} 11^{\prime} 20^{\prime \prime}$ & $116^{\circ} 38^{\prime} 53^{\prime \prime}$ & $8-28-2001$ & 0 & & \\
\hline 13344700 & Deep Creek tributary near Potlatch, ID & $47^{\circ} 01^{\prime} 27^{\prime \prime}$ & $116^{\circ} 53^{\prime} 01^{\prime \prime}$ & $8-29-2001$ & 0 & & \\
\hline 13346450 & South Fork Palouse River near Moscow, ID & $46^{\circ} 42^{\prime} 41^{\prime \prime}$ & $116^{\circ} 58^{\prime} 46^{\prime \prime}$ & $8-29-2001$ & 0 & & \\
\hline
\end{tabular}


